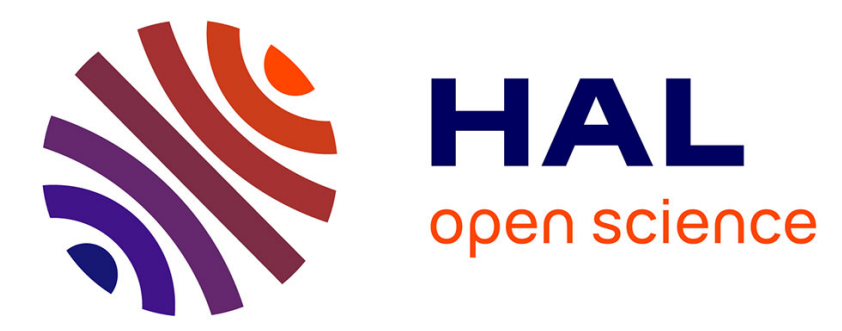

\title{
Meaningful Blurs: the Sources of Repetition-based Plurals in ASL
}

Philippe Schlenker, Jonathan Lamberton

\section{To cite this version:}

Philippe Schlenker, Jonathan Lamberton. Meaningful Blurs: the Sources of Repetition-based Plurals in ASL. Linguistics and Philosophy, 2021, 10.1007/s10988-020-09312-9 . hal-03516722

\section{HAL Id: hal-03516722 \\ https://hal.science/hal-03516722}

Submitted on 7 Jan 2022

HAL is a multi-disciplinary open access archive for the deposit and dissemination of scientific research documents, whether they are published or not. The documents may come from teaching and research institutions in France or abroad, or from public or private research centers.
L'archive ouverte pluridisciplinaire HAL, est destinée au dépôt et à la diffusion de documents scientifiques de niveau recherche, publiés ou non, émanant des établissements d'enseignement et de recherche français ou étrangers, des laboratoires publics ou privés. 


\title{
Meaningful Blurs: the Sources of Repetition-based Plurals in $\mathrm{ASL}^{*}$
}

\author{
Philippe Schlenker** \\ (Institut Jean-Nicod, CNRS; New York University) \\ Jonathan Lamberton
}

To appear in Linguistics \& Philosophy

\begin{abstract}
In several sign languages, plurals can be realized with unpunctuated or punctuated repetitions of a noun, with different semantic implications; similar repetitionbased plurals have been described in some homesigns and silent gestures. Unpunctuated repetitions often get approximate 'at least' readings while punctuated repetitions typically correspond to 'exactly' readings. The prevalence of these mechanisms could be thought to be a case in which Universal Grammar does not just specify the abstract properties of grammatical elements, but also their phonological realization, at least in the visual modality. We explore an alternative in which punctuated and unpunctuated repetitions arise from general properties of iconic representations. On an empirical level, we argue that in ASL (American Sign Language), punctuated and unpunctuated repetitions are unlikely to be an exclusively grammatical mechanism, as they can be found with purely iconic (word-free) representations. On a theoretical level, we argue for a modular account with three components. First, repetition-based plurals can create a simplified pictorial representation. Second, unpunctuated repetitions give rise to pictorial vagueness, resolved by way of quantification over precisifications. Third, a pragmatic process involving strategic reasoning maps these vague representations onto a set of candidate linguistic meanings, including some 'at least' plural readings that are best expressed by unpunctuated repetitions.
\end{abstract}

Keywords: sign language semantics, iconicity, plurals, unpunctuated repetitions, punctuated repetitions, pictorial semantics, iconic semantics, super semantics

\footnotetext{
* Authors' acknowledgments: we are very grateful to three reviewers for Linguistics \& Philosophy for extraordinarily constructive and helpful comments, which led to several improvements. Editor Patrick Grosz was also very helpful. Kathryn Davidson and Benjamin Spector made numerous suggestions and objections which helped improve the paper. P.S.'s acknowledgments: For discussion and references, I am indebted to Kathryn Davidson, Paul Egré, Carlo Geraci, Gabe Greenberg, Jeremy Kuhn. Special thanks to Benjamin Spector for particularly illuminating discussions of the pragmatic part, and to the audience of the LINGUAE seminar (Paris, April 23, 2020) for helpful suggestions. For judgments on onomatopoeias in English, I am very grateful to Brian Buccola, Jonah Katz, Jeremy Kuhn, Nathan Klinedinst, Matthew Mandelkern, and Lyn Tieu. I also received much input on comics from Jonathan Bonhomme and Claire Schlenker. Many thanks to Lucie Ravaux for checking all averages and for putting the raw scores in the Excel file made available in the Supplementary Materials, as well as for working on the bibliography.

Authors' contributions: Philippe Schlenker initiated this research, constructed all examples in consultation with Jonathan Lamberton, and developed the analysis. Jonathan Lamberton was the ASL consultant for the initial phase of the work. When it was written, he checked or provided transcriptions and translations, and discussed important aspects of the analysis. Any theoretical discussion among co-authors occurred only after the data were collected and the first version of the article was written, and primarily by email because the authors were not on the same continent. The separation between the two phases of the work was intended to minimize the risk the 'theoretical contamination' of sign language judgments.

Grant acknowledgments: This research received funding from the European Research Council (ERC) under the European Union's Horizon 2020 research and innovation programme (grant agreement No 788077, Orisem, PI: Schlenker). Research was conducted at Institut d'Etudes Cognitives, Ecole Normale Supérieure - PSL Research University. Institut d'Etudes Cognitives is supported by grants ANR-10-IDEX-0001-02 and FrontCog ANR-17EURE-0017.

** Institut Jean-Nicod (ENS - EHESS - CNRS), Département d'Etudes Cognitives, Ecole Normale Supérieure, Paris, France; PSL Research University ; New York University, New York.
} 


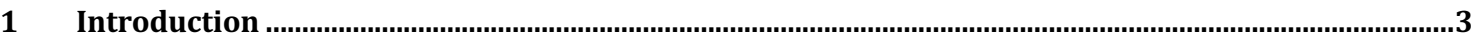

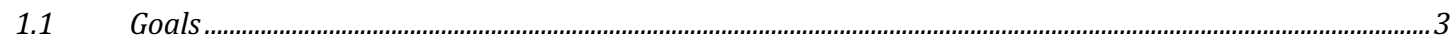

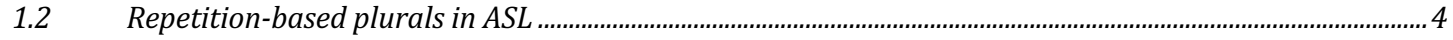

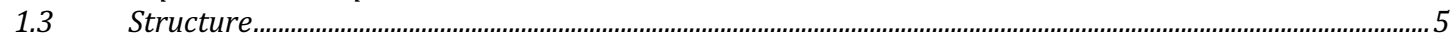

$1.4 \quad$ Transcription conventions and elicitation methods............................................................................................... 6

2 Grammatical vs. iconic interpretation of an ambiguous constructions .....................................................8

3 Comparing repetitions of nouns and of purely iconic signs.................................................................10

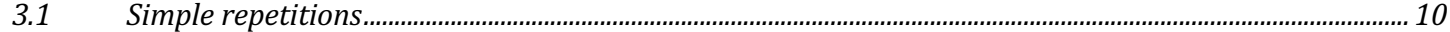

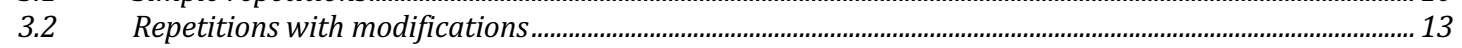

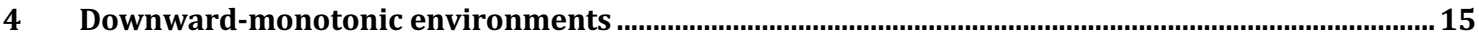

4.1 'Exactly' readings in downward-monotonic environments ....................................................................... 15

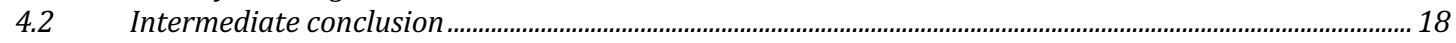

$4.3 \quad$ Outlook: logical and pictorial conditions ............................................................................................................ 18

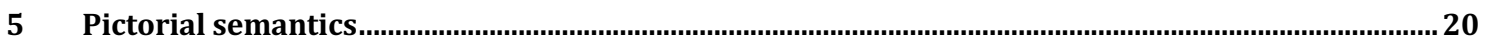

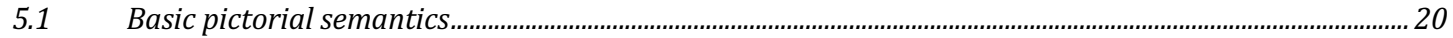

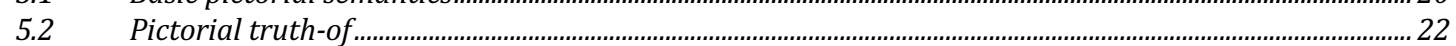

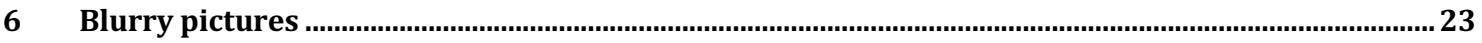

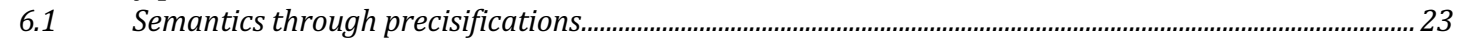

6.2 Using bivalent tolerant truth for blurry pictures ......................................................................................... 25

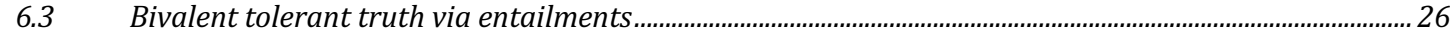

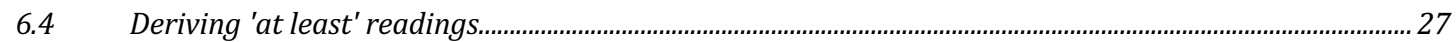

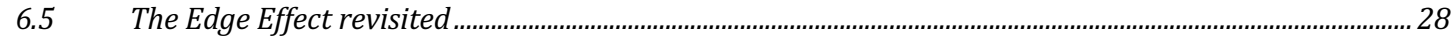

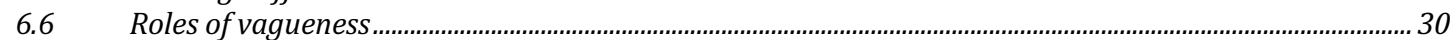

$7 \quad$ Revisiting the truth conditions of ASL repetition-based plurals .............................................................30

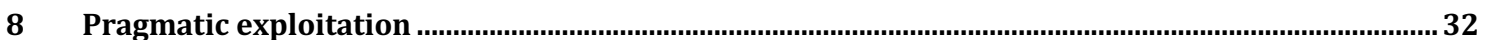

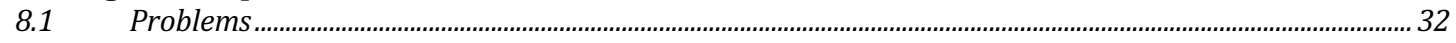

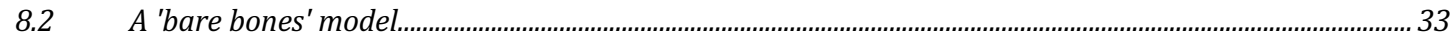

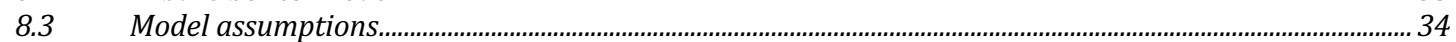

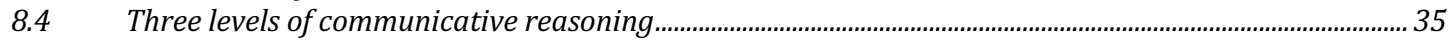

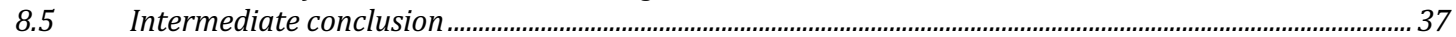

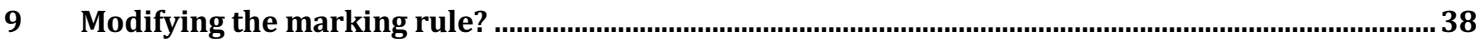

$9.1 \quad$ A problem

$9.2 \quad$ An iconic solution

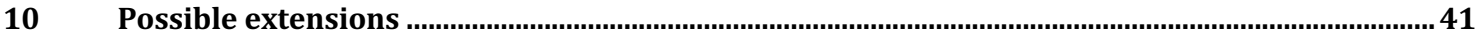

$10.1 \quad$ Connection with experimental data on classifier predicates ........................................................................ 41

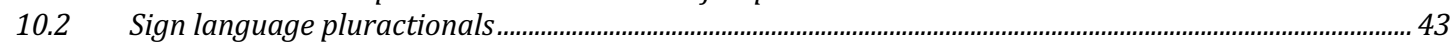

$10.3 \quad$ Repetition-based plurals and pluractionals in gestures...................................................................................... 43

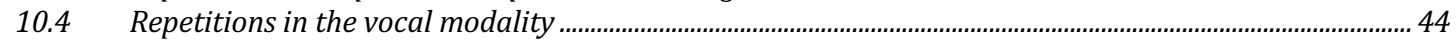

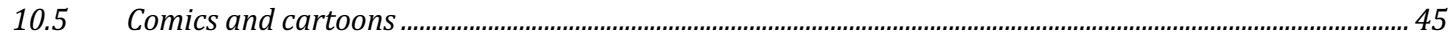

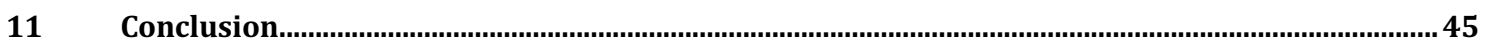

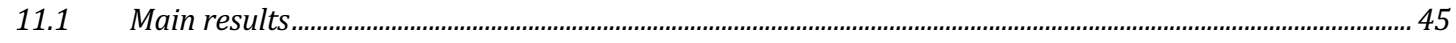

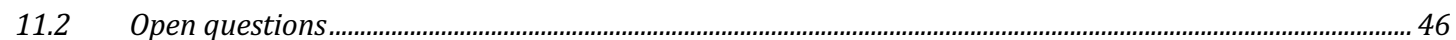

Appendix I. Blurry pictures: truth via entailments vs. bivalent tolerant truth............................................47

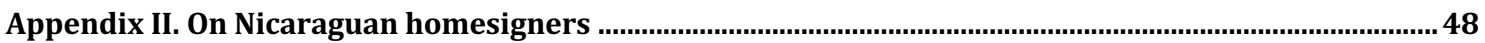

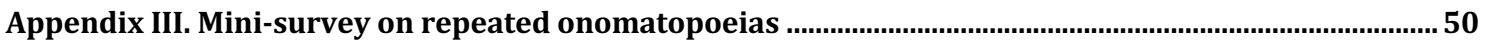

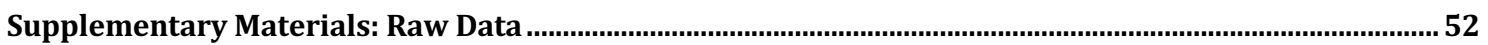

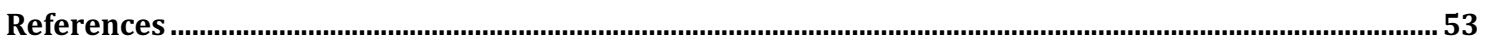




\section{Introduction}

\subsection{Goals}

Plurals have been argued to be realized by two kinds of repetitions in several sign languages, as well as in some homesigns and in some pro-speech (= word-replacing) gestures (Pfau and Steinbach 2006, Coppola et al. 2013, Abner et al. 2015, Schlenker and Lamberton 2019, Schlenker, to appear a). Punctuated repetitions are made of discrete, clearly separable iterations of the same nominal sign in different parts of signing space, and they are usually associated with precise quantitative conditions, corresponding to the number of iterations. Unpunctuated repetitions involve iterations with shorter and less clear breaks between them, which makes them less distinct and typically harder to count; they are associated with imprecise quantitative conditions and are often compared to spoken language plurals. The existence of both types of repetitions in a homesigner investigated with experimental means by Coppola et al. 2013 was striking because his hearing mother didn't use such repetitions: this was a grammatical-like device that he had invented. Similarly, non-signers have been argued to understand repetition-based plurals in gestures, with a distinction between punctuated and unpunctuated ones (Schlenker and Lamberton 2019, Schlenker, to appear a). The ability of some homesigners to produce and of some non-signers to understand different kinds of repetition-based plurals with little or no input raises a question: what is the source of repetition-based plurals in signs and in gestures?

One possibility is that, in this particular area, Universal Grammar does not just specify the abstract properties of some grammatical features (such as person and number), but also their phonological realization. On this view, Universal Grammar specifies a mapping between the grammatical form of plurals and certain patterns of repetition. This might not be a unique case: several properties of signs have been argued to appear (in far less sophisticated form) in some gestures (e.g. Schlenker, to appear a). Nor is the assumption that innate mechanisms specify the meaning of some gestures outlandish: as argued in recent research, ape communication involves a number of innate gestures whose meaning is stable within species and to some extent shared across related species (Graham et al. 2018, Byrne et al. 2017). Still, a purely grammatical (noniconic) version of this view is hard to maintain, at least for ASL (American Sign Language). Schlenker and Lamberton 2019 show that repetition-based plurals can be associated with detailed iconic conditions: in their data, the construction doesn't just specify the number of objects involved, but also their spatial arrangement; for instance, three punctuated iterations of TROPHY arranged as a triangle refer to a group of three trophies arranged in this fashion. They make similar (if preliminary) claims about repetitions in gestures. For ASL, they argue that the distinction between unpunctuated and punctuated repetitions should in the end be viewed in an iconic fashion, but they do not go into the details of how the iconic process works. Still, a weakened version of the grammatical view remains a live option: it could be that the distinction between unpunctuated and punctuated repetitions is grammatical and specified by Universal Grammar, even though the iconic component isn't so specified (in part because it is gradient and modifiable at will).

We develop an alternative to these grammatical theories and argue that the realization of punctuated and unpunctuated repetitions originates in iconic cognition. Specifically, we propose that repetition-based plurals in signs (and probably gestures) are an area in which iconic semantics and pragmatic reasoning conspire to yield a grammatical effect. Grammar plays a role at an initial stage in providing a Logical Form for the surrounding expressions (e.g. by way of existential quantification over pluralities), and at the last stage in providing some candidate meanings for iconic expressions. But the mapping between these candidate meanings and various forms is determined by iconic and pragmatic principles.

Our main empirical argument is that internal to ASL, unpunctuated and punctuated repetitions can have the same types of implications for normal signs and for entirely iconic, wordfree representations, including in atypical cases in which each iteration differs from the preceding 
one (e.g. to represent objects of increasing size). ${ }^{1}$ On a theoretical level, we propose to start from an iconic semantics based on geometric projections, as in Greenberg's $(2013,2019)$ analysis of pictorial semantics. This immediately yields precise quantitative conditions for punctuated repetitions. The case of unpunctuated repetitions is more complex because it involves pictorial vagueness. We analyze it within a precisification-based 'tolerant' logic, one that makes a representation true just in case one of its precisifications is (our semantics is a 'bare bones' bivalent version of systems discussed in Cobreros et al. 2012). This semantics for blurry pictures then feeds into a pragmatic process mapping pictures to candidate meanings through a process of pragmatic enrichment with a strategic component, following the general spirit of the Rational Speech Act Model (RSA, e.g. Goodman and Frank 2016, Franke and Jäger 2016, and Bergen et al. 2016).

The significance of these results for semantic theory is twofold. Substantively, they suggest that as logical a category as plurality may be realized through iconic means: this is an area in which logic and iconicity are intertwined at the heart of language. Methodologically, this is to our knowledge the clearest case so far in which the details of Greenberg's pictorial semantics are crucial to develop a semantic analysis of an uncontroversially linguistic phenomenon. This justifies an expansion of the semanticist's toolbox - in fact, several extensions, since our data will require that pictorial semantics be combined with vagueness and a mechanism of pragmatic enrichment.

\subsection{Repetition-based plurals in ASL}

In a typological study, Pfau and Steinbach 2006 note that "reduplication and zero marking appear to be two basic pluralization strategies attested in all sign languages that have been investigated so far". Schlenker and Lamberton 2019 develop an analysis of repetition-based plurals in ASL, with a special focus on three types of repetitions, which they illustrate with (1) (with acceptability judgments on a 7-point scale at the beginning of each example).

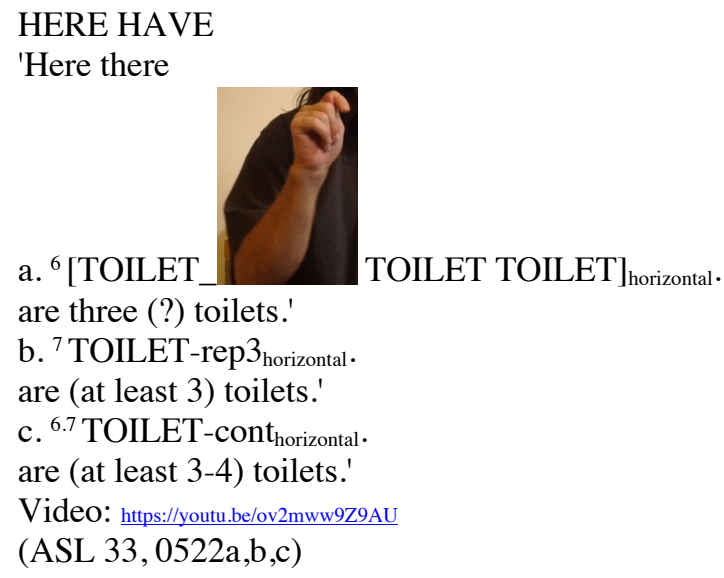

As Schlenker and Lamberton 2019 write,

(1)a is a punctuated repetition, with three horizontal iterations of the word TOILET, realized as a trembled $T$ of the manual alphabet, whose first iteration is illustrated (...). Characteristic is the fact that the hand goes down between the three iterations, which makes them easy to individuate: the $T$ handshape disappears or goes back to a neutral position between iterations. In (1)b, there are three iterations as well, but realized a bit faster, and in an unpunctuated fashion, characterized by the fact that the hand only partly goes down

\footnotetext{
${ }^{1}$ By 'word-free representations', we mean improvised gestural representations identified by the native ASL consultant (and co-author) as corresponding to no conventional lexical form. We explore cases of repetition with modification to make it even less likely that a grammatical operation is at work (since to our knowledge standard pluralization does not involve such modifications of a lexical sign).
} 
between the iterations: the $T$ handshape only partially disappears between them. The realization can be much faster in other examples, making it hard to count the iterations, and highlighting the contrast with punctuated repetitions (which is rather subtle in (1)). Finally, in (1)c a continuous repetition is illustrated; the hand does not go down at all between the iterations, and these are faster, more numerous, and very hard to count.

In one of their final analyses, summarized in (2), all repetition-based plurals have the same semantic core. First, bare nouns are assumed to be true of atoms and non-atoms like, as is made plausible by the fact a bare noun can have a plural reading, especially when combined with a numeral. This justifies Condition (i), which states that a repeated noun $N$ should hold true of the denoted group. ${ }^{2}$ In addition, however, repetition-based plurals crucially come with an iconic component, defined in Condition (ii), and they also have the ability to create new singular discourse referents, as seen in Condition (iii); the latter is motivated by the fact that in restricted cases individual iterations are made available for further anaphoric uptake.

(2) Schlenker and Lamberton 2019, "Improved Analysis 1": semantics of punctuated, unpunctuated and continuous repetitions without pluralization, but with the assumption that count terms are true of atoms and non-atoms

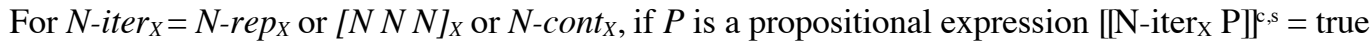
iff for some group $\mathrm{d}$,

(i) $[[\mathrm{N}]]^{\mathrm{c}, \mathrm{s}[\mathrm{X} \rightarrow \mathrm{d}]}(\mathrm{d})=$ true, and

(ii) $N$-iter iconically represents d given $\mathrm{c}$, and

(iii) $[[\mathrm{P}]]^{\mathrm{c}, \mathrm{s}\left[\mathrm{X} \rightarrow \mathrm{d}, \mathrm{x}_{1} \rightarrow \mathrm{d}_{1}, \ldots, \mathrm{x}_{\mathrm{n}} \rightarrow \mathrm{d}_{\mathrm{n}}\right]}=$ true, where $x_{l}, \ldots, x_{n}$ are discourse referents made available by the iconic semantics of $N$-iter, and $x_{1}, \ldots, x_{n}$ respectively denote $\mathrm{d}_{1}, \ldots, \mathrm{d}_{\mathrm{n}}$ (which are parts of $\mathrm{d}$ ).

This analysis is underspecified as to the difference between punctuated and unpunctuated repetitions. We take up this problem in the present piece, varying two parameters that are somewhat conflated in this earlier analysis: (i) whether the iterations are realized slowly or quickly, and (ii) whether the hand goes down between the iterations. In clear earlier cases, unpunctuated repetitions were realized quickly and without the hand going down, while punctuated repetitions were realized slowly and with the hand going down. Since we are interested in the precise source of these readings, it will be helpful to distinguish these two properties. For this reason, we will be talking of separated vs. unseparated iterations depending on whether the hand goes down, and slow or fast iterations depending on their speed. A clear punctuated repetition would thus be slow and separated, a clear unpunctuated repetition fast and unseparated. As we will see, the traditional terminology of punctuated vs. unpunctuated makes excellent sense in the end, because what seems to matter for most (though not all) purposes is whether the iterations are presented as easy or hard to count. (We leave out continuous repetitions from the present discussion. They were primarily used by Schlenker and Lamberton to investigate iconic properties of mass nouns, but some of their continuous repetitions applied to count nouns are arguably similar to the fast unseparated iterations we study in this piece.)

\section{$1.3 \quad$ Structure}

The rest of this article is organized as follows. After laying out our transcription conventions and elicitation methods (Section 1.4), we introduce our initial finding: one and the same construction may be ambiguous between a repetition-based plural and a purely iconic use of a repetition, but in such cases the grammatical and the iconic constructions give rise to broadly similar inferences (Section 2). We then turn to simpler and unambiguous paradigms, systematically comparing the pluralization of words and the repetition of purely iconic and improvised manual representations, and arguing that punctuated vs. unpunctuated repetitions yield the same semantic differences

\footnotetext{
${ }^{2}$ Without the assumption that nouns hold true of atoms and non-atoms alike, one would need to posit an operation of pluralization of the noun before it can hold true of non-atoms.
} 
across the two types (Sections 3-4). We develop our analysis in stages, starting with a pictorial semantics augmented with a precisification-based account of vague representations (Section 56), which can be integrated with a standard semantics for nominals (Section 7). We then consider an improvement that relies on a mechanism of pragmatic enrichment: while on purely iconic grounds unpunctuated repetitions are very unlikely to have 'at least' readings, this possibility is exploited and thus made likely through strategic communicative reasoning (Section 8). We refine our analysis in Section 9, and extend it to different data types (including gestures and onomatopoeias) in Section 10, before summarizing our conclusions and open questions (Section $11)$.

Since the argument and the analysis have several moving parts, we provide a table to help the reader gain a bird eye's view of the proposal.

(3) Structure of the proposal

\begin{tabular}{|c|c|c|c|}
\hline \multirow{3}{*}{$\begin{array}{l}\text { Empirical } \\
\text { analysis }\end{array}$} & \multirow[t]{2}{*}{$\begin{array}{l}\text { Iconic vs. grammatical uses } \\
\text { of repetitions }\end{array}$} & $\begin{array}{l}\text { One and the same construction is ambiguously } \\
\text { linguistic or purely iconic, and } \\
\text { punctuated/unpunctuated repetitions yield the same } \\
\text { reading types across the linguistic and iconic cases. }\end{array}$ & Section 2 \\
\hline & & $\begin{array}{l}\text { Punctuated/unpunctuated repetitions as well as } \\
\text { repetitions with modifications have similar semantic } \\
\text { effects across the linguistic and purely iconic cases. }\end{array}$ & Section 3 \\
\hline & $\begin{array}{l}\text { 'Exactly' vs. 'at least' } \\
\text { readings }\end{array}$ & $\begin{array}{l}\text { Punctuated repetitions can have 'exactly' readings', } \\
\text { unpunctuated repetitions can have 'at least readings': } \\
\text { argument from downward-monotonic environments. }\end{array}$ & Section 4 \\
\hline \multirow{4}{*}{$\begin{array}{l}\text { Core } \\
\text { Theory }\end{array}$} & Basic pictorial semantics & $\begin{array}{l}\text { A basic pictorial semantics yields an appropriate } \\
\text { account of punctuated repetitions. }\end{array}$ & Section 5 \\
\hline & $\begin{array}{l}\text { Pictorial semantics with } \\
\text { vagueness }\end{array}$ & $\begin{array}{l}\text { With a vague component, a pictorial semantics can } \\
\text { yield an account of unpunctuated repetitions. }\end{array}$ & Section 6 \\
\hline & $\begin{array}{l}\text { Integrating logical and } \\
\text { pictorial semantics }\end{array}$ & $\begin{array}{l}\text { The logical and the iconic components can be } \\
\text { integrated by way of quantification over pluralities } \\
\text { that must resemble the repeated element. }\end{array}$ & Section 7 \\
\hline & Pragmatic enrichment & $\begin{array}{l}\text { Even if the set of precisifications needed to derive } \\
\text { 'at least' readings is extremely unlikely, this } \\
\text { possibility is exploited for communicative purposes. }\end{array}$ & Section 8 \\
\hline \multirow[t]{2}{*}{ Extensions } & $\begin{array}{l}\text { Refining the pictorial } \\
\text { semantics: improving the } \\
\text { marking rule }\end{array}$ & $\begin{array}{l}\text { Slow punctuated repetitions can have 'at least' } \\
\text { readings; this can be derived using a more } \\
\text { discriminating marking rule. }\end{array}$ & Section 9 \\
\hline & $\begin{array}{l}\text { Adding cost to the } \\
\text { pragmatic analysis? }\end{array}$ & $\begin{array}{l}\text { In some homesigners, 'at least' readings are } \\
\text { preferably realized with } 2-3 \text { (rather than } n \text { ) } \\
\text { repetitions. This might require (future) extensions } \\
\text { that take into account the cost of repetitions. }\end{array}$ & Appendix II \\
\hline
\end{tabular}

\subsection{Transcription conventions and elicitation methods ${ }^{3}$}

In the following, sign language sentences are glossed in capital letters, as is standard. Translations were chosen to reflect, to the extent possible, the inferential judgments that were given. Inferential questions appear with the examples. Raw data can be consulted in the Supplementary Materials.

A suffixed locus, as in WORD- $i$, indicates that the word points towards locus i (a position of signing space associated with a discourse referent). $I X$ (for 'index') is a pointing sign towards a locus, while POSS is a possessive; they are glossed as $I X-i$ and POSS $i$ if they point towards (or 'index') locus $i$; the numbers 1 and 2 correspond to the position of the signer and addressee respectively. $I X-i$ is a standard way of realizing a pronoun corresponding to locus $i$, but we also use $I X$ without a suffixed locus when it is repeated. Agreement verbs include loci in their realization - for instance the verb 1-GIVE-2 starts out from the first person locus 1 and targets the second person locus 2; it means that the signer gives something to the addressee.

\footnotetext{
${ }^{3}$ This part is adapted from Schlenker and Lamberton 2019.
} 
In the literature and in (1), the suffix -rep is used for unpunctuated repetitions, and in such cases -rep3, -rep $4,-$ rep $5, \ldots$ indicate that there are $3,4,5, \ldots$ iterations. When relevant, we add a subscript indicating the shape of the repetition, e.g. - rep 3 horizontal for a horizontal repetition (whether in a straight line or as a horizontal arc), -rep $3_{\text {triangle }}$ for a triangular-shaped repetition. The suffix -cont is used for continuous repetitions, and subscripts may be used as well to indicate

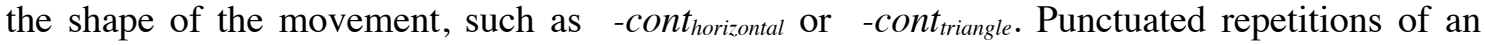
expression WORD are encoded as [WORD WORD WORD] if they involve three iterations of that expression; [WORD WORD WORD] horizontal and [WORD WORD WORD] triangle provide information about the shape of the repetition.

In this piece, we refine these descriptions. We use -unsep 3 for 3 unseparated repetitions, -sep 3 for 3 separated repetitions, and the subscripts fast and slow for fast and slow repetitions respectively. We also encode as a subscript the collective shape formed by the repetitions. For instance, we use o-unsep $4_{\text {fast-circular }}$ to encode 4 unseparated fast iterations of an o-like shape, and these 4 shapes collectively form a circle. Notational conventions will be introduced or refined as we go. We do not encode non-manual expressions, which remained as constant as possible within sentences of a given paradigm (i.e. appearing within the same numbered example).

The consultant (and co-author) is a Deaf, native signer of ASL (of Deaf, signing parents). ${ }^{4}$ Elicitation was conducted using the 'playback method', described for instance in Schlenker et al. 2013, Schlenker 2014, Schlenker and Lamberton 2019. It involved repeated quantitative acceptability judgments (1-7, with 7 = best), as well as well as inferential judgments, obtained in two ways: by way of open questions, and by way of quantitative judgments of inferential strength (1-7, with $1=$ no inference and $7=$ strongest inference). Judgments of inferential strength have become standard in experimental pragmatics, and have proven useful in ASL fieldwork (Schlenker, to appear b) ${ }^{5}$. For readability, only average judgments are given (complete quantitative judgments are given when there is more than a 2-point difference in the judgments obtained for a given sentence). In summary tables, we use gray shades for sentences that are degraded (average acceptability at or below 4), as inferential judgments in these cases might not be very informative. Specialists are invited to consult the raw data in the Supplementary Materials when relevant. References such as ASL, 35, 1492, 3 judgments at the end of paradigms crossreference the ASL video (here video 34, 1492) and indicate the number of judgment tasks.

One important disclaimer is in order. As mentioned in Schlenker, to appear b, one might worry that our sentences are assessed by the very signer that produced them. It is clear that our results can only bear on the idiolect of a particular individual. But the work we conduct on this idiolect is arguably more rigorous than is common in non-experimental fieldwork, since (i) we distinguish the production phase from the judgment phase, and (ii) we provide multiple quantitative data points that make it possible to assess the stability (or lack thereof) of the judgments through time.$^{6}$ It goes without saying that exploring these data with further consultants would be of great importance in the future.

\footnotetext{
${ }^{4}$ We use the term 'consultant' when we discuss information obtained in elicitation sessions (and thus prior to writing the article).

${ }^{5}$ As Cremers and Chemla 2017 write, that graded inferential judgments "may help detect otherwise hidden effects". This was borne out for our ASL consultant (who has years of experience with quantitative acceptability judgments): he noted in the past that the inferential part of his task was made easier, not harder, by using quantitative judgments of inferential strength. Without these, he had to reflect at length about how to categorize judgments of intermediate strength; the quantitative method allowed for less arbitrary decisions in such cases.

${ }^{6}$ This method should of course be assessed rigorously in the future, but this holds of other elicitation methods as well - and ours has the advantage of transparency (since the raw written data are made available in the Supplementary Materials).
} 


\title{
2 Grammatical vs. iconic interpretation of an ambiguous construction
}

We now argue that punctuated and unpunctuated repetitions alike have the same kinds of semantic implications in purely iconic (word-free) representations as they do in linguistic environments, which suggests that they are based on a cognitive mechanism that is not purely linguistic.

We start with a paradigm in which, depending on the context, the same sequence can be interpreted as a grammatical construction involving a repetition-based plural, or as a purely iconic (word-free) representation. The grammatical construction is slightly different from the ones we've discussed up to this point: instead of involving the repetition of a noun, it includes the repetition of a pointing sign associated with a noun. Specifically, the construction of interest (in (4) below) is HOUSE followed by three pointing signs. A grammatical use of the latter construction implies the presence of several houses, with different quantitative conditions depending on the realization of the repetitions. For instance, with three slow separated iterations, the implication is that there are four houses, i.e. one for the initial occurrence of HOUSE, and an additional one for each pointing sign. But the very same sequence can also be understood in a completely different way. Specifically, if the context specifies that I have a contemporary drawing with nothing but houses followed by dots, the three 'pointing signs' can be taken to be iconic representations of dots (importantly, this does not involve the word DOT, which would be realized by way of fingerspelling or a classifier, for instance ${ }^{7}$ ). In our case, the word HOUSE is taken to represent a singular house, and three slow separated iterations of the iconic representation of a dot indicate that there are three dots next to the house in the drawing. We call these three iterations 'word-free' because there is no lexical form at all used here: this is, to the best of our knowledge, an improvised gestural and iconic representation of a dot (using one's finger), similar to one that a non-signer could produce.

Using the ambiguous construction in (4) allows for a very minimal comparison between a grammatical and an iconic use of a repetition: the consultant thus assessed one and the same video twice in each judgment task, just changing the context in which it was evaluated, thereby switching the interpretation from 'several houses' to 'one house followed by several dots'.

(4) Context 1: I have a contemporary drawing with nothing but houses.

Context 2: I have a contemporary drawing with nothing but houses followed by dots.

\author{
POSS-1 DRAWING \\ 'My drawing has \\ a. HOUSE. \\ has a house.' \\ b. HOUSE IX-unsep $3_{\text {fast-horizontal }}$. \\ at least three houses.' $\quad$ (Context 1) \\ a house next to at least four dots. ${ }^{18} \quad$ (Context 2) \\ c. HOUSE IX-unsep $3_{\text {slow-horizontal }}$. \\ exactly four houses.' $\quad$ (Context 1) \\ a house next to exactly three dots.' $\quad$ (Context 2)
}

\footnotetext{
${ }^{7}$ For some examples, see the discussion by William Vicars at http://www.lifeprint.com/asl101/pages-signs/d/dots.htm (retrieved on November 6, 2019).

${ }^{8}$ One should not overinterpret the 'at least four' inference found in this case and reported in (6). One might expect instead an 'at least (approximately) three' reading because there are three iterations. But as one can check by watching the video, the number of iterations is just very difficult to count in this case. In Context 1 , there is an 'at least four' reading alongside the 'at least three' reading, as can also be seen in (6). Still, we don't know why Context 1 and Context 2 do not give rise to entirely parallel results - nor whether the difference is significant (in view of the limitations of our methods).
} 
d. HOUSE IX-sep $3_{\text {fast-horizontal }}$.

(deviant in both contexts)

e. HOUSE IX-sep3 $3_{\text {slow-horizontal }}$.

exactly four houses.'

(Context 1)

a house next to exactly three dots.' (Context 2)

(ASL, 35, 1440, 3 judgments per context; anonymized video: https://youtu.be/njmMqpCshY4)

A word should be said about the inferential judgments. They were of two kinds: open judgments about the number of relevant objects found in the drawing; and judgments of inferential strength pertaining to 'exactly $\mathrm{n}$ ', 'at least $\mathrm{n}$ ' and 'approximately $\mathrm{n}$ ' readings (with $7=$ strongest endorsement), with questions displayed in (5). ${ }^{9}$ We only report the latter, quantitative judgments in the text. But it can be checked in the Supplementary Materials that these did not miss anything essential of the answers to the open questions. In the grammatical case, HOUSE followed by three slow separated iterations of $I X$ yielded an inference that there were exactly 4 houses (not exactly 3 ), presumably because each occurrence of $I X$ referred to an additional house. In our summary table in (6), we encoded how many objects the repeated term referred to: it would be 3 in this case (since this is the contribution made by the repeated pointing sign). (Note that the number of fast, unseparated iterations is very hard to count on the video for (4)b, hence the contrast between the 'at least 3 ' and the 'at least 4' readings need not be very telling; see fn. 8.)

(5) Inferential questions (sample; see the Supplementary Materias for Meaning 2)

Meaning 1: What do you infer about the number of (Context 1) houses found (Context 2) dots

found in the contemporary drawing? (provide an estimate, e.g. $n$, more than $n$, between $m$ and $n$, etc)

Meaning 3: About the objects mentioned in Meaning 1:

Context 1: How strongly do you infer that there were (i) exactly 4 ? (ii) approximately 4 ? (iii) at

least 4 ? (iv) at least 5 ?

Context 2: How strongly do you infer that there were (i) exactly 3 ? (ii) approximately 3 ? (iii) at least 3 ? (iv) at least 4 ?

(6) Acceptability and inferential judgments (Meaning 3) obtained for (4)

\begin{tabular}{|c|c|c|c|c|c|c|}
\hline \multirow{2}{*}{$\begin{array}{l}\text { Examples } \\
(\mathrm{ASL}, \underline{35,1440)}\end{array}$} & \multirow[t]{2}{*}{ Context } & \multirow[t]{2}{*}{ Acceptability } & \multicolumn{4}{|c|}{ Number of objects that the repetitions stand for } \\
\hline & & & $=3$ & \pm 3 & $\geq 3$ & $\geq 4$ \\
\hline \multirow[t]{2}{*}{ a. HOUSE } & 1 grammatical & 7 & 1 & 1 & 1 & 1 \\
\hline & 2 iconic & $5.7(3,7,7)$ & 1 & 1 & 1.3 & 1 \\
\hline \multirow[t]{2}{*}{ b. HOUSE IX-unsep $3_{\text {fast }}$} & 1 grammatical & 6.7 & 1.3 & 2.7 & 5.3 & 4.7 \\
\hline & 2 iconic & 5 & 1 & 1.3 & 2 & 6.3 \\
\hline \multirow[t]{2}{*}{ c. HOUSE IX-unsep $3_{\text {slow }}$} & 1 grammatical & 7 & 6 & 3.7 & 2 & 1 \\
\hline & 2 iconic & 6.7 & 6 & 3 & 1.7 & 1 \\
\hline \multirow[t]{2}{*}{ d. HOUSE IX-sep3 fast $_{\text {f }}$} & 1 grammatical & 2.7 & 4 & 5 & 3.7 & 2.3 \\
\hline & 2 iconic & 3.3 & 3.7 & 5 & 3.7 & 1.7 \\
\hline \multirow[t]{2}{*}{ e. HOUSE IX-sep3 $3_{\text {slow }}$} & 1 grammatical & 7 & 5.7 & 3 & 3.3 & 1 \\
\hline & 2 iconic & 7 & 5.7 & 2.3 & $\begin{array}{l}2.3 \\
(1 ; 1 ; 5)\end{array}$ & 1 \\
\hline
\end{tabular}

The results reported in (6) are striking: whether it is interpreted as a pluralized form of the pointing sign, or as a purely iconic (gestural) representation of multiple dots, the repeated pointing gives rise to rather similar quantitative inferences (modulo the difference, mentioned at the outset, that in the grammatical interpretation all quantities must be incremented by 1 due to the presence of the noun HOUSE). The control in (4)a is just interpreted as involving one house and no dots (an unrepeated noun is often interpreted by default as involving singularity; when co-

\footnotetext{
${ }^{9}$ Note that the inferential possibilities were clearly understood with their pragmatic implications. For instance, in (4)e there was a high endorsement of the 'exactly three' reading and a lower endorsement of the 'approximately 3 ' and 'at least three' readings, no doubt because these were interpreted to exclude the more informative 'exactly 3 ' situations.
} 
occurring with numerals, the situation radically changes, of course). The other examples all involve 3 iterations of pointing. In (4)b, they are realized as unseparated and fast, hence a typical unpunctuated repetition. When interpreted grammatically, the iterations yield an inference that there are at least 3 or 4 houses in addition to the one represented by the noun. When interpreted iconically, they yield an inference that there are at least 4 dots (thus this is not exactly the same inference as in the grammatical case, but close enough). When realized as unseparated but slow, the 3 iterations yield an inference to the existence of exactly 3 (additional) houses, or of exactly 3 dots. Here and throughout, signing the repetitions separated but roughly at the same speed as unseparated fast repetitions is deviant (this just seems inconvenient to realize); since all such examples are severely degraded, we will not discuss the inferences they give rise to. When realized as separated and slow, the repetitions primarily give rise to an inference about the existence of exactly 3 (additional) houses, or exactly three dots.

In sum, a paradigm constructed to be ambiguous between a grammatical and an iconic reading gives rise to the same inferences for punctuated vs. unpunctuated repetitions across the two readings. This suggests that the inferences triggered by different types of repetitions might arise outside of purely grammatical cases.

\section{Comparing repetitions of nouns and of purely iconic signs}

\subsection{Simple repetitions}

Our initial example had the advantage of allowing for the assessment of the very same signs on two interpretations, one grammatical and one purely iconic, depending on the context. But this forced us to use a slightly more involved construction than a standard plural. In a normal plural construction, HOUSE would be repeated to yield a plural meaning. In the grammatical interpretation of the case we studied, a plural meaning was obtained with HOUSE followed by the iteration of the pointing sign - a crucial device in order to get the ambiguity we wanted. In this section, we explore simpler and unambiguous paradigms without a pointing sign, of two sorts: some involve the repetition of a normal sign, others that of a purely iconic representation.

The repetitions we consider are arranged in a circle. This choice was made to help bring out semantic differences among the examples. Our initial worry was this: plurals can easily get existential readings. Take for instance 4 punctuated iterations of a circle on a line, in the context of a drawing (assume for concreteness that these are purely iconic, word-free representations, although the problem is similar for words). This could be taken to mean that it is possible to find four circles in the drawing; this reading wouldn't preclude the presence of further circles on the line. So one would have to ask about inferences about the number of circles on the relevant part of the line, which yields complications (e.g. there could be a continuation of the line that the signer decided not to represent). We sought to limit the problem by representing the repetitions in a circle, where it might be less likely that there were further objects that the signer decided to omit, as it might seem more natural to omit part of a line on a drawing than to omit part of a circle (as we will see in Section 4, this ploy was not entirely successful, in a way that might be informative).

We start with the repetition of a word, here the $G$ of the manual alphabet. It does not in any way resemble the printed letter $G$, as can be seen in (7), and we will often refer to it as $F 7^{10}$. In other words, it is a word that happens to refer - non-iconically - to a letter.

\footnotetext{
${ }_{10}$ There are different possible realizations of ASL $G$. This one is close to what is represented in (7) (the drawing is from https://www.realsasl.com/learn-south-african-sign-language/144-south-african-sign-language-alphabet-abc, retrieved on October 17, 2019).
} 
(7) Sign for G (signed high, in (8)a, as a controlf for circular repetitions that follow)

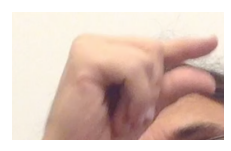

To facilitate comparison with purely iconic cases, we used a context pertaining to a contemporary abstract drawing, which may contain letters in some cases, and geometric shapes in others.

As in all other cases, there were open and closed, quantitative inferential questions. We report judgments of inferential strength for the Meaning 3 in (9), and we henceforth write acceptability judgments as superscripts at the beginning of the relevant sentences. (It is somewhat difficult to count the manual G's in the 'unseparated fast' condition, a remark that parallels an observation we made about the paradigm in (4).)

Notation: $G$-unsep $4_{\text {fast-circular }}$ refers to 4 fast unseparated iterations of the manual $G$ (i.e. $\$$ ) arranged as a circle.

(8) Context: I have a contemporary abstract drawing

POSS-1 DRAWING

'My drawing contains
a. ${ }^{7} \mathrm{G}$.
a G.'
b. ${ }^{7}$ G-unsep $4_{\text {fast-circular }}$
at least 4 or 5 G's (arranged in a circular fashion).'
c. ${ }^{7} \mathrm{G}$-unsep $4_{\text {slow-circular }}$
exactly $4 \mathrm{G}$ 's(arranged in a circular fashion).'
d. ${ }^{4} \mathrm{G}-\mathrm{sep} 4_{\text {fast-circular }}$.
(deviant)
e. ${ }^{7}$ G-sep $4_{\text {slow-circular. }}$.
4 / at least 4 G's (arranged in a circular fashion).'
(ASL, 35, 1492, 3 judgments; anonymized video: https:///youtu.be//GXOawdkEa-s)

(9) Inferential questions (sample; here too, see the Supplementary Materials for Meaning 2)

Meaning 1: What do you infer about the number of shapes in the contemporary drawing? (provide an estimate, e.g. $\mathrm{n}$, more than $\mathrm{n}$, between $\mathrm{m}$ and $\mathrm{n}$, etc)

Meaning 3: About the objects mentioned in Meaning 1: How strongly do you infer that there were

(i) exactly 4? (ii) approximately 4? (iii) at least 4? (iv) at least 5?

(10) Inferential judgments (= Meaning 3) obtained for (8)

\begin{tabular}{|l|l|l|l|l|}
\hline \multirow{2}{*}{$\begin{array}{l}\text { Examples } \\
(\text { ASL }, 35,1492)\end{array}$} & \multicolumn{4}{|l|}{ Number of objects that the repetitions stand for } \\
\cline { 2 - 5 } & $=4$ & \pm 4 & $\geq 4$ & $\geq 5$ \\
\hline a. G & 1 & 1 & 1 & 1 \\
\hline b. G-unsep4 fast-circular $_{\text {f }}$ & 1.7 & 3.3 & 5.3 & 5.7 \\
\hline c. G-unsep4 $4_{\text {slow-circular }}$ & 5.7 & 3.3 & 1.7 & 1 \\
\hline d. G-sep4 fast-circular & 2 & 3.7 & $4.3(6 ; 3 ; 4)$ & $4.3(5 ; 2 ; 6)$ \\
\hline e. G-sep4 $4_{\text {slow-circular }}$ & 4.7 & 3.7 & 5 & 2 \\
\hline
\end{tabular}

With one exception, the generalizations are the same as in Section 2: 4 unseparated iterations of $G$ signed quickly give rise to the inference that there are at least 4 or at least $5 \mathrm{G}$ 's in the circle (it is worth noting that the fast unseparated iterations are genuinely hard to count, which makes the contrast between at least 4 and at least 5 moot). By contrast, 4 slow unseparated iterations give rise to the inference that there were exactly (or possibly approximately) 4 . Fast separated iterations are degraded, as before. One difference relative to the paradigm in (4) is that slow 
separated iterations might be ambiguous, meaning exactly/approximately 4 or at least 4 (in (4)e the separated slow repetitions only gave rise to an 'exactly' reading). We come back to an explanation of this ambiguity in Section 9.

We turn to a minimally different case in which an iconic manual shape represents a small circle, as in (11). This is close in shape to a classifier used for small objects, but here it is just interpreted to represent a circle, in an iconic fashion. ${ }^{11}$

(11) Iconic representation of a small circle (signed high, in (12)a, as a controlf for circular repetitions that follow)

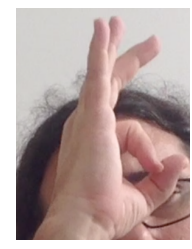

(12) Context: I have a contemporary abstract drawing.

POSS-1 DRAWING

'My drawing contains

a. ${ }^{7} \mathrm{o}$.

a little circle.'

b. ${ }^{7}$ o-unsep $4_{\text {fast-circular. }}$.

at least 5 little circles (arranged in a circular fashion).'

c. ${ }^{7} \mathrm{O}$-unsep $4_{\text {slow-circular. }}$.

exactly 4 little circles (arranged in a circular fashion).'

d. ${ }^{3.7} \mathrm{o}$-sep 4 fast-circular.

(deviant)

e. ${ }^{7} \mathrm{O}$-sep $4_{\text {slow-circular. }}$.

exactly 4 little circles (arranged in a circular fashion).'

(ASL, 35, 1466, 3 judgments; anonymized videos: https://youtu.be/d-7CwleawUM)

(13) Inferential judgments obtained for (12)

\begin{tabular}{|c|c|c|c|c|}
\hline \multirow{2}{*}{$\begin{array}{l}\text { Examples } \\
\text { (ASL, } \underline{35,1466)})\end{array}$} & \multicolumn{4}{|c|}{ Number of objects that the repetitions stand for } \\
\hline & $=4$ & \pm 4 & $\geq 4$ & $\geq 5$ \\
\hline a. o & 1 & 1 & 1 & 1 \\
\hline b. o-unsep4 $4_{\text {fast-circular }}$ & 1 & 3.3 & 3 & 5.7 \\
\hline c. o-unsep4 $4_{\text {slow-circular }}$ & 5.7 & 3.3 & 2 & 1 \\
\hline d. o-sep4 $4_{\text {fast-circular }}$ & 4.3 & 5 & 2.7 & 1.3 \\
\hline e. o-sep4 $4_{\text {slow-circular }}$ & 5.3 & 2.7 & $3.3(1 ; 4 ; 5)$ & 1.7 \\
\hline
\end{tabular}

Rather similarly to the repetition of $G$ in (8), unseparated fast iterations give rise to an 'at least 5 ' reading (with $G$, the 'at least 4 ' inference was strong as well). As in earlier cases, the difference between 'at least 4 ' and 'at least 5 ' in unseparated fast repetitions should not be overinterpreted: it is just very hard to count the number of occurrences in this case, and thus the distinction between 4 and 5 unseparated fast iterations would probably be hard to detect. With slow unseparated iterations, 'exactly 4 ' is the dominant reading. Fast separated iterations are, as always, deviant. Slow unseparated iterations yield an 'exactly 4 ' reading (the 'approximately 4 ' reading is a bit

${ }^{11}$ As Lamberton notes, the circle represented on the fingers in (11) is slightly bigger than the shape he would use for the small object classifier (for him, the diameter of the circle represented inside the thumb and index finger might be $25-50 \%$ smaller in the classifier use). In addition, the small object classifier would be accompanied by a facial expression. 
weaker than with the example involving $G$, as are judgments for the 'at least 4' reading, which are also unstable in this case).

In sum, there is a strong similarity between the effects of various types of repetitions with the manual $G$ on the one hand and with an iconic manual representation on the other: unseparated fast iterations give rise to 'at least' readings, unpunctuated slow iterations to 'exactly' readings', unseparated slow iterations are less clear (with an 'exactly' reading, but further interpretations as well in some cases, including an 'at least' reading); unseparated fast iterations are always deviant.

\subsection{Repetitions with modifications}

Our argument has a flaw, however: we can't exclude the possibility that our iconic representations are words created on the spot, which are then treated according to the grammatical rules of the language. In other words, it could be that all the interesting action is in the transformation of an improvised iconic sign into a word, not in the repetition process that pluralizes it. We attempt to address this issue by modifying the grammatical process itself: instead of a standard repetition, we investigate a repetition with a change of shape, with each iteration larger than the preceding one. We do not know of descriptions of grammatical repetitions that involve such changes of shape from one iteration to the next.

We start with the iconic case, involving again the manual representation of a small circle, as in (11). But here each new iteration will have a larger opening than the preceding one, with the inference that the circles increase in size. (In the rest of this section, we do not provide the inferential questions, because they are similar to those used in earlier examples; they can be found in the Supplementary Materials.)

Notation: We encode the fact that the iterations are of increasing size by way of the symbol $<$. Thus o-<unsep $4_{\text {fast-circular }}$ refers to 4 fast unseparated iterations of little circles of increasing size, arranged in a circular fashion.

(14) Context: I have a contemporary abstract drawing.

POSS-1 DRAWING

'My drawing contains

a. ${ }^{7}$ o.

a little circle.'

b. ${ }^{5.7} \mathrm{o}-<$ unsep $4_{\text {fast-circular. }}$.

at least 5 little circles of increasing size (arranged in a circular fashion).'

c. ${ }^{6} \mathrm{O}$-< unsep4 $4_{\text {slow-circular. }}$.

exactly 4 little circles of increasing size (arranged in a circular fashion).'

d. ${ }^{3} \mathrm{o}-<\mathrm{sep} 4_{\text {fast-circular. }}$.

(deviant)

e. ${ }^{6.7} \mathrm{O}-<$ sep $4_{\text {slow-circular. }}$

exactly 4 little circles of increasing size (arranged in a circular fashion).'

(ASL, 35, 1464, 3 judgments; anonymized video: https://youtu.be/xc7pUsLAKY8)

(15) Inferential judgments obtained for (12)

\begin{tabular}{|l|l|l|l|l|}
\hline \multirow{2}{*}{$\begin{array}{l}\text { Examples } \\
\text { ASL, 35, 1464) }\end{array}$} & \multicolumn{4}{|l|}{ Number of objects that the repetitions stand for } \\
\cline { 2 - 5 } & $=4$ & \pm 4 & $\geq 4$ & $\geq 5$ \\
\hline a. o & 1 & 1 & 1 & 1 \\
\hline b. o-<unsep4 fast-circular & 1.3 & 3.3 & 2.7 & 5.7 \\
\hline c. o-<unsep4 $4_{\text {slow-circular }}$ & 6 & 2.3 & 1.7 & 1 \\
\hline d. o-<sep4 fast-circular & 4.7 & 5 & 2.3 & 1.3 \\
\hline e. o-<sep4 $4_{\text {slow-circular }}$ & 5.3 & $3.3(2 ; 3 ; 5)$ & $3.3(1 ; 4 ; 5)$ & 1.3 \\
\hline
\end{tabular}


As before, the representation with fast separated iterations is deviant. While the slow separated iterations are acceptable, the unseparated iterations are a bit less so, possibly because changing the hand shape in this semi-continuous fashion is less than natural. In any event, the same general contrasts are found as in our earlier paradigms. First, unseparated fast repetitions give rise to an 'at least' reading - in this case: at least 5 rather than at least 4 , which need not be surprising because (as mentioned before) the number of iterations is just very hard to count. Unseparated slow repetitions yield an 'exactly four' reading because of the four iterations. Separated slow repetitions also primarily give rise to an 'exactly four' reading, with greater uncertainty about the 'approximately four' and 'at least four' readings - a point we revisit in Section 9 (we will predict an ambiguity in this case).

It is interesting to compare this purely iconic example to that of a $G$ repeated with a change of shape: the initial shape is a $G$ of the manual alphabet, i.e. $\$$, and the rectangle formed by the thumb and index finger is gradually opened from one iteration to the next, leading to an $L$ shape at the end, i.e. ${ }^{12}$, as illustrated in (16).

(16) First and fourth manual shape appearing in (17)e (proportions are not preserved)

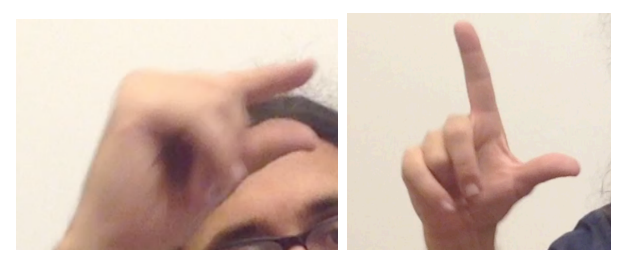

General contrasts are the same as in the iconic case, but the interpretation of the shape itself is of some interest: due to the change of shape from one iteration to the next, a purely iconic interpretation becomes salient, whereby the drawing contains geometric shapes (rather than letters) akin to the manual shapes displayed. Still, as explicitly noted by the consultant, this purely iconic interpretation need not fully preclude a partly iconic interpretation whereby the shapes represent letters: a $G$ becoming larger and then becoming an $L$ (but see the Supplementary Materials for details $\left.{ }^{13}\right)$.

Context: I have a contemporary abstract drawing

POSS-1 DRAWING

'My drawing contains

a. ${ }^{7} \mathrm{G}$.

a G.'

b. ${ }^{6} \mathrm{G}-<$ unsep $4_{\text {fast-circular. }}$.

approximately 4 shapes of increasing size ranging from from 7 to (arranged in a circular fashion).'

c. ${ }^{6.3} \mathrm{G}-<$ unsep4 $4_{\text {slow-circular }}$.

exactly 4 shapes of increasing size ranging from from to (arranged in a circular fashion).'

d. ${ }^{2} \mathrm{G}-<$ sep 4 fast-circular.

(deviant)

e. ${ }^{6.7} \mathrm{G}-<$ sep 4 slow-circular .

${ }^{12}$ Our drawing (from the same source as in fn. 10) is not consistent between ${ }_{7}$ and the former is given from the signer's perspective, the latter from the addressee's perspective (see the pictures in (16) for the 'real' addressee perspective).

${ }^{13}$ One helpful remark made by the consultant (= [JL 19.10.10]) was phrased as follows: "The signs used might represent abstract shapes rather than actual letters, particularly since the intermediate shapes are clearly not letters. However, the first and last shapes strongly invoke the letters $G$ and $L$ and that rules for me." 
exactly 4 shapes of increasing size ranging from from $\$$ to (arranged in a circular fashion).'

(ASL, 35, 1494, 3 judgments ; anonymized video: https://youtu.be/XYwDcywuw-w)

(18) Inferential judgments obtained for (17)

\begin{tabular}{|l|l|l|l|l|}
\hline \multirow{2}{*}{$\begin{array}{l}\text { Examples } \\
\text { (ASL, } 35,1494)\end{array}$} & \multicolumn{4}{|l|}{ Number of objects that the repetitions stand for } \\
\cline { 2 - 5 } & $=4$ & \pm 4 & $\geq 4$ & $\geq 5$ \\
\hline a. o & 1 & 1 & 1 & 1 \\
\hline b. o-<unsep4 $4_{\text {fast-circular }}$ & 3 & 5 & $4(3 ; 3 ; 6)$ & $3(2 ; 2 ; 5)$ \\
\hline c. o-<unsep4 $4_{\text {slow-circular }}$ & 6 & 2 & 1.7 & 1 \\
\hline d. o-<sep4 fast-circular & 4.3 & 5 & 4.3 & 3 \\
\hline e. o-<sep4 $4_{\text {slow-circular }}$ & 5.3 & 3.3 & 4 & 1.7 \\
\hline
\end{tabular}

In sum, we have established that purely iconic representations can be iterated by way of punctuated and unpunctuated repetitions in the same way as normal words, and with comparable semantic effects. On the one hand, purely iconic (and improvised) representations can enter in standard patterns of repetitions, including with some 'at least' readings that are characteristic of plurals. On the other hand, the repetition process itself can be modulated in iconic ways, with each repetition larger than the preceding one - a process that deviates from grammatical repetitions. When applied to a noun (here the letter name $\quad$ ), the repetition with modification brings out an iconic reading pertaining to the very shape displayed, but the number and realization of the repetitions yield the same quantitative effects as in other cases..$^{14}$

\section{Downward-monotonic environments}

One important question is whether the 'exactly' readings noted above are an intrinsic component of the literal meaning of the examples, or arise due to an implicature, by competition with other forms. Unembedded sentences cannot decide the issue. To give an example from English, if the numeral three means at least three, as on many standard treatments, it is expected to give rise to an 'exactly three' reading in unembedded cases due to competition with four. To decide whether its literal meaning has an exactly three component, we must embed the numeral in downwardmonotonic environments, where implicatures are unlikely to arise. This is so because, even for theories that allow implicatures to be computed locally (e.g. Chierchia et al. 2012), the contribution of an expression in such environments is usually its literal meaning. ${ }^{15}$

\section{1 'Exactly' readings in downward-monotonic environments}

We consider sentences of the form if $p$, I will give you $\$ 20$, with plural-based repetitions within $p$, and inferential questions pertaining to numbers that would force the signer to give the addressee $\$ 20 . I F$-clauses normally prevent implicatures from being computed locally, ${ }^{16}$ so we expect to be

\footnotetext{
${ }^{14}$ Since we do not know of other studies of such repetitions with modifications, our discussion leaves several questions open, including (i) how they semantically compare to standard plurals in various environments, and (ii) how they interact with other categories, such as adjectives.

${ }^{15}$ The case of numerals in English turns out to be complicated: as discussed in Spector 2013, one of the leading current theories is that numerals have an at least reading (in particular under downward-monotonic environments), but that they associate with exhaustivity operators more freely than other constructions, hence the appearance of exactly readings even in downward-monotonic environments. We revisit this question in relation to ASL repetition-based plurals at the end of Section 4.2.

${ }^{16}$ The reason is that $I F$-clauses behave like downward-monotonic environments, which both license Negative Polarity Items such as ever and suspend implicatures, as illustrated in (i):
}

(i) a. If you ever go to China, you should visit Shanghai.

b. If you invite Robin or Sam, I'll give you \$20.

$=>$ if you invite Robin and Sam, I'll give you \$20. 
able to assess in this way the literal meaning of the constructions. ${ }^{17}$ Since we are interested in the iconic semantics of repetitions, we focus on a context involving look like (a shape), which highlights this iconic component. Our first paradigm involves repetitions of the manual $G$, i.e. $\sum$, and some of the results replicate what we saw before, but now with an argument that we are assessing the literal meaning of the iconic representation rather than the result of an implicature. The inferential judgments were intended to test the quantitative condition that would force the signer to give the addressee $\$ 20$, hence the formulations in (19).

(19) Inferential questions (sample)

Meaning 1: What is number of shapes in the contemporary drawing that will force the signer to give the addressee $\$ 20$ ? (provide an estimate, e.g. $n$, more than $n$, between $m$ and $n$, etc)

Meaning 3: About the objects mentioned in Meaning 1 and assuming the drawing's properties other than number of shapes fit the parameters of the signed sentence: How strongly do you infer that the signer is committed to giving the addressee $\$ 20$ in case there are

(i) exactly 4 (ii) approximately 4 (iii) at least 4 (iv) at least 5

of these objects?

(20) Context: The addressee has a contemporary abstract drawing

IF POSS-2 DRAWING LOOK-LIKE IX-1 1-GIVE-2 20 \$.

'If your drawing looks like this, with ..., I'll give you $\$ 20$.'

a. ${ }^{7} \mathrm{G}$

a $\mathrm{G}$ towards the top

b. ${ }^{7}$ G-unsep $4_{\text {fast-circular }}$

at least $4 \mathrm{G}^{\prime}$ s arranged in a circular fashion

c. ${ }^{7}$ G-unsep 4 slow-circular

exactly $4 \mathrm{G}^{\prime} \mathrm{s}$ arranged in a circular fashion

d. ${ }^{3.3} \mathrm{G}$-sep $4_{\text {fast-circular }}$

(deviant)

e. ${ }^{7}$ G-sep4 $4_{\text {slow-circular }}$

exactly (or at least?) 4 G's arranged in a circular fashion

(ASL, $\underline{35,1560}, 3$ judgments)

\begin{tabular}{|c|c|c|c|c|}
\hline \multirow{2}{*}{$\begin{array}{l}\text { Examples } \\
(\mathrm{ASL}, \underline{35,1560)})\end{array}$} & \multicolumn{4}{|c|}{ Number of objects that the repetitions stand for } \\
\hline & $=4$ & \pm 4 & $\geq 4$ & $\geq 5$ \\
\hline a. $\mathrm{G}$ & $3.7(1 ; 4 ; 6)$ & $3.7(1 ; 4 ; 6)$ & $3.7(1 ; 4 ; 6)$ & $3.7(1 ; 4 ; 6)$ \\
\hline b. G-unsep $4_{\text {fast-circular }}$ & 6.7 & 6.7 & 6.7 & 7 \\
\hline c. G-unsep4 $4_{\text {slow-circular }}$ & 7 & 2.7 & 1.3 & 1 \\
\hline d. G-sep4 fast-circular $_{\text {r }}$ & 6.3 & 6 & $4.3(2 ; 4 ; 7)$ & $4.7(1 ; 6 ; 7)$ \\
\hline e. G-sep4 $4_{\text {slow-circular }}$ & 7 & $5.3(7 ; 5 ; 4)$ & $4.7(7 ; 5 ; 2)$ & $4(7 ; 4 ; 1)$ \\
\hline
\end{tabular}

Unseparated fast repetitions yield inferences consistent with an at least 4 reading, with the result that no matter whether the drawing has 4 or more than 4 G's, the signer is committed to giving the addressee \$20. This is similar to one would obtain with the following English sentence: If

${ }^{17}$ Some care is needed, however, as any semantic differences might be erased if the context somehow facilitates an existential reading. Concretely: suppose one predicate, call it $=4$, means 'exactly 4 circles', and the other, call it $\geq 4$, means 'at least 4 circles'. If the target sentence is analyzed as in (i), with an existential quantifier within the $I F$-clause and a distributive predicate, the semantic difference between the two expressions will be erased. Some of the examples we investigated in our fieldwork are arguably like this, and we focus in the text on those that are not.

$$
\begin{aligned}
& \text { a. If } \exists x=4(x) \& x \text { can be seen, I'll give you } \$ 20 . \\
& \text { b. If } \exists x \geq 4(x) \& x \text { can be seen, I'll give you } \$ 20 \text {. }
\end{aligned}
$$


your drawing displays at least $4 G^{\prime}$ 's arranged as a circle, I'll give you $\$ 20$. Any number of appropriate G's at or above 4 would commit the signer to giving the $\$ 20$. One potential difference in the ASL sentence is that there might be a vague component about the threshold, so it might be (clumsily) paraphrased as 'at least approximately 4 G's'; this might explain the high endorsement in the \pm 4 column in (20)a.

Importantly, unseparated slow repetitions have an 'exactly' reading: it is now only in case the drawing has exactly $4 \mathrm{G}$ 's that the signer must give the addressee $\$ 20$. This suggests that this 'exactly' reading is not the result of a standard implicature, which would be expected not to arise within the scope of the $I F$-clause. But this leaves open two possibilities, discussed in greater detail below: one (our preferred option) is that this 'exactly' reading stems from the literal meaning of the construction due to its iconic semantics; the other is that this 'exactly' reading stems from a local implicature akin to that of numerals, which can be computed in the scope of $I F$-clauses.

In addition, a difference arises in this context between slow unseparated and slow separated repetitions: the latter seem to be ambiguous, with an 'exactly 4 ' and an 'at least 4' reading, and unstable inferential judgments (unlike the other cases, where judgments are mostly stable). In fact, the example in (20)a with a lone $G$ towards the top might raise a similar problem: there seems to be uncertainty as to whether the drawing should contain a $G$ towards the top, and nothing else, or at least a $G$ towards the top.

Related conclusions can be drawn from the same paradigm with iconic shapes replacing letters, as in (21).

(21) Context: The addressee has a contemporary abstract drawing

IF POSS-2 DRAWING LOOK-LIKE __, IX-1 1-GIVE-2 20 \$.

'If your drawing looks like this, with ..., I'll give you \$20.'

a. ${ }^{7} \mathrm{o}$

a little circle towards the top

b. ${ }^{7}$ o-unsep $4_{\text {fast-circular }}$

at least 4 little circles arranged in a circular fashion

c. ${ }^{7}$ o-unsep $4_{\text {slow-circular }}$

exactly 4 little circles arranged in a circular fashion

d. ${ }^{3.7} \mathrm{o}$-sep $4_{\text {fast-circular }}$

(deviant)

e. ${ }^{7}$ o-sep $4_{\text {slow-circular }}$

exactly (or at least?) 4 little circles arranged in a circular fashion

(ASL, $\underline{35,1562,3 \text { judgments) }}$

\begin{tabular}{|l|l|l|l|l|}
\hline \multirow{2}{*}{$\begin{array}{l}\text { Examples } \\
(\text { ASL, 35, 1562) }\end{array}$} & \multicolumn{4}{|l|}{ Number of objects that the repetitions stand for } \\
\cline { 2 - 5 } & $=4$ & \pm 4 & $\geq 4$ & $\geq 5$ \\
\hline a. o & $4(1 ; 4 ; 7)$ & $4(1 ; 4 ; 7)$ & $4(1 ; 4 ; 7)$ & $4(1 ; 4 ; 7)$ \\
\hline b. o-unsep4 fast-circular $_{\text {r }}$ & 6.7 & 6.7 & 6.7 & 7 \\
\hline c. o-unsep4 slow-circular & 7 & 2.3 & 1.3 & 1 \\
\hline d. o-sep4 fast-circular & 7 & 5.7 & $4.7(2 ; 5 ; 7)$ & $5(1 ; 7 ; 7)$ \\
\hline e. o-sep4 $4_{\text {slow-circular }}$ & 7 & $5.3(7 ; 4 ; 5)$ & $5(7 ; 5 ; 3)$ & $4.3(7 ; 5 ; 1)$ \\
\hline
\end{tabular}

Here too, fast unseparated repetitions are compatible with an 'at least' reading. A clear 'exactly' reading arises with unseparated slow repetitions. But once again there is a distinction between these and separated slow repetitions, which seem to allow both for an 'exactly' and for an 'at least' reading. Some of our unembedded examples, for example in (17), displayed contrasts that went in the same direction (clear 'exactly' reading for unseparated slow iterations, additional possibilities for separated slow iterations); others did not display this contrast. This might be because an 'at least' reading was obfuscated by the presence of an implicature ('at least 4, but not at least 5 , hence exactly 4 '). 


\subsection{Intermediate conclusion}

It is worth pausing to state our main generalizations.

(22) Readings obtained with repetition-based plurals

a. In all environments, 4 unseparated fast iterations give rise to an 'at least' reading ('at least 4' or 'at least 5 ' - the latter might particularly arise when the iterations are particularly hard to count).

b. In all environments, 4 unseparated slow iterations give rise to an 'exactly 4 ' reading.

c. In unembedded environments, 4 separated slow iterations give rise to an 'exactly 4 ' reading, and they seem to be ambiguous between an 'exactly' and an 'at least' reading under $I F$-clauses.

d. In all cases, 4 separated fast iterations are degraded.

The contrast between separated and unseparated slow repetitions is a theoretical challenge. There are two directions one could explore.

Analysis I. Local implicatures in downward-monotonic environments: First, one could posit that both constructions have an 'at least' literal meaning, but that in the case of slow unseparated repetitions it gets enriched by default through a local implicature, computed within the scope of the $I F$-clause, whereas this is optional in the case of slow separated repetitions. This line of analysis raises two issues: (1) Why are locally computed implicatures possible or even nearobligatory in this case, despite the fact that the environment is downward-monotonic? (2) And why is there a difference in this respect between these two types of repetitions?

The first question might be less thorny than it looks, as 'exactly' readings also arise with garden-variety numerals in English inside if-clauses (e.g. If Sam is 14 years old, he must be difficult to deal with). The theory of numerals proposed by Spector 2013 is precisely that numerals differ from other scalar expressions in that they more easily associate with embedded exhaustivity operators, including in downward-monotonic environments. But the second question is much trickier, as it is entirely unclear why one construction should give rise to embedded implicatures in downward-monotonic environments while the other doesn't. ${ }^{18}$

Analysis II. A semantic difference between unseparated and separated repetitions: As an alternative, one could posit that the literal meaning of unseparated and separated slow repetitions is different: the former should have an 'exactly' reading, while the latter should be ambiguous between an 'exactly' and an 'at least' reading. We will develop a proposal along these lines in Section 9.

We turn to an explanation of these patterns, based on a pictorial semantics (outlined in Section 5) combined with a theory of pictorial vagueness (Section 6). This will yield an initial derivation of the desired truth conditions (Section 7), which will be refined through a pragmatic procedure (Section 8) and a revision of one aspect of the pictorial semantics (Section (10). (The last point will provide an argument for Analysis II.)

For most of the discussion, we will assume that the crucial factor is whether iterations are easy or hard to count, which justifies going back to the traditional terminology of 'punctuated' (and thus easy to count) vs. 'unpunctuated' repetitions (harder to count). But we will refine the analysis Section 9, where we try to explain a potential difference between unseparated and separated slow iterations.

\subsection{Outlook: logical and pictorial conditions}

In the rest of this piece, we will successively develop a simple pictorial semantics (to deal with punctuated repetitions) and a pictorial semantics with vagueness (to deal with unpunctuated repetitions). This pictorial component will then be integrated with a logical semantics involving

${ }^{18}$ This problem pertains to the contrast between two types of 'easy to count' repetitions, slow unseparated and slow separated ones. A further issue for this theory is why unpunctuated repetitions, i.e. fast unseparated repetitions, should fail to give rise to an embedded implicature. 
quantification over pluralities that must resemble the repetition (in the precise sense of pictorial semantics). Various important refinements, including in the pragmatics, will be developed after the core semantics has been developed.

To illustrate, take the punctuated repetition in (8)c, repeated as (23)a. It involves four punctuated (here: unseparated slow) iterations of the manual $G$ (i.e. $\$$ ), arranged in a circular fashion. We will posit the Logical Form in (23)a', where the boxed part is an existential quantifier over pluralities of G's resembling the repetition, and introducing a variable $X$ (ranging over pluralities).

(23) a. POSS-1 DRAWING G-unsep4 $4_{\text {slow-ircular. }}$

'My drawing contains exactly 4 G's (arranged in a circular fashion).'

a'. G-unsep4 $4_{\text {slow-circular } x}$ [my-drawing has $\mathrm{X}$ ]

b. $\left[\left[\left(a^{\prime}\right)\right]\right]^{c, s}=$ true iff $\ldots$ for some group $d$,

(i) $\left[[G]^{c, s[X \rightarrow d]}(d)=\right.$ true, i.e. $d$ is a group of $G^{\prime} s$, and

(ii) G-unsep $4_{\text {slow-circular }}$ is (pictorially) true of d, and

(iii) $[$ my-drawing has $\mathrm{X}]]^{c, s[X \rightarrow d]}=$ true, i.e. my drawing has d.

The schematic truth conditions we will derive are given in (23)b. (23)b(i), (iii) are standard logical conditions: the existential statement is made true only if one can find a group $\mathrm{d}$ which is made of G's, and satisfies [my-drawing has X] when the variable $\mathrm{X}$ denotes d. On the other hand, the condition (23)b(ii) will arise from a pictorial semantics: we will consider $G$-unsep $4_{\text {slow-circular }}$ as a picture, or rather a picture part, which can be true of certain objects, and we will require that $d$ should be one such (plural) object. Our pictorial semantics will explicate in detail what it means for a picture part to be true of an object, and thus the schematic truth conditions in (23)b will in the end aggregate logical and pictorial conditions.

The details of the pictorial semantics will greatly matter at several junctures, but one point should already be kept in mind. In a purely iconic repetition (e.g. of the o-shape), both the individual shape and the arrangement of the repetitions resemble the denotation; by contrast, in a word-based repetition (e.g. of the manual $G$ ), the linguistic symbol need not resemble what it denotes, although the arrangement of the symbols provides information about the arrangement of the denoted group. This distinction will be handled in due course by modulating the marking rule, i.e. the rule by which pixels of a picture are marked in view of what projects onto them. To anticipate: we will take the marking rule for the case of word repetitions to be semi-iconic and semi-symbolic. We will take pixels to be the elementary picture parts that can be filled in accordance with marking rules, but for simplicity we will posit that there are very large pixels in the cases of interest. More precisely, on the assumption that the picture/signing space on the left is made of 9 (very large!) pixels, we will specify that a will appear in a pixel just in case a $G$ from the drawing projects onto it, as illustrated in (24). (The case of purely iconic repetitions will be simpler, with a pictorial component and no symbolic component at all.)

(24) A semi-iconic and semi-symbolic marking rule: the 7 's do not resemble their denotations (G's on a drawing), but their arrangement does provide iconic information about the arrangement of the denoted group.

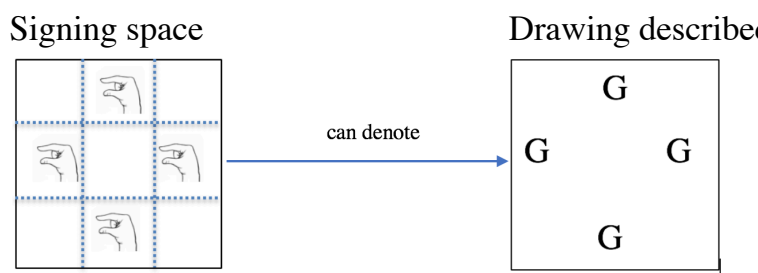




\section{$5 \quad$ Pictorial semantics}

We turn to the first component of our analysis, which is based on a semantics for pictorial representations. But to see where we are going, we must first outline how a pictorial semantics works.

\subsection{Basic pictorial semantics}

Greenberg 2013, 2019 develops an explicit semantics for pictures, crucially based on the notion of a geometric projection: for him, a picture is true in a world $\mathrm{w}$ relative to a viewpoint $\mathrm{v}$ if $\mathrm{w}$ projects onto the picture relative to v. In Greenberg's words (2019),

a simple type of PERSPECTIVE PROJECTION is illustrated in [(25)a]. Here we begin with a concrete 3dimensional region of spacetime (possible or actual), which I'll think of as a possible world. In the example below, the world contains only a cube. Next, a PROJECTION SOURCE is located within the space of the world. A projection source is thought of simply as a geometric point in space and time. This in turn defines a system of PROJECTION LINES, which link each point in the world to the source. Finally, a PICTURE PLANE is introduced into this spray of lines, and they are used to map spatially distributed features of the scene back to surface features of the picture plane itself - in this case, the lines of the line drawing. The result of such a projection is displayed at right below.

(25) Examples of a projection-based semantics for pictures (Greenberg 2019a)

a. Perspective projection

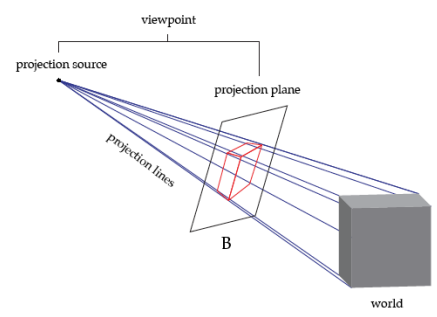

b. Parallel projection

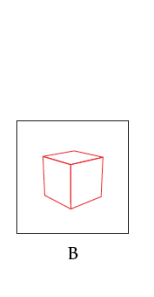

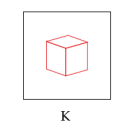

As summarized in Schlenker 2019, the viewpoint includes both the projection source and the position of the picture. The relevant projection lines are given by the system of projection assumed: a system of "linear projection" is represented in (25)a, and is "characterized by the fact that the projection source is a point, to which all projection lines converge" (Greenberg 2019a); other systems can be used, for instance "parallel projection", illustrated in (25)b, whereby "the projection lines, rather than converging on a single point, all run perpendicular to the projection source, hence parallel to one another" (Greenberg 2019a).

Simplifying somewhat, this allows Greenberg to define the truth of a picture in a world relative to a viewpoint, as in (26).

(26) Pictorial truth (Greenberg 2019a)

A picture $\mathrm{P}$ is true in world $\mathrm{w}$ relative to viewpoint $\mathrm{v}$ along the system of projection $\mathrm{S}$ iff ${ }^{19} \mathrm{w}$ projects to $\mathrm{P}$ from viewpoint $\mathrm{v}$ along $\mathrm{S}$, or in other words:

$\operatorname{projs}_{\mathrm{s}}(\mathrm{w}, \mathrm{v})=\mathrm{P}$

As Greenberg 2019a notes, a projection system has two components: a "projection condition", which "establishes the systematic spatial relationship between points in the scene and points on the picture plane, as embodied by projection lines" (parallel projection in the case

19 Greenberg 2019a is committed to the 'only if' part but not necessarily to the 'if' part, because further principles might enrich the content of a picture. In case some situations are plausible while others are extremely implausible, the latter might be excluded from consideration when computing the truth conditions of pictures. (Thanks to G. Greenberg, p.c., for discussion of this point.) 
illustrated in (25)); and a "marking" rule, which "associates properties of the scene with types of mark on the picture plane, following the spatial distribution specified by the first stage".

We will consider two simplified marking conditions, pertaining to black-and-white pictures, or equivalently pictures manually sketched with handshapes appearing in various parts of a 2-dimensional space. One marking condition is purely iconic, and corresponds to a case discussed by Greenberg 2019a, in which "the features associated with each point in the array correspond to those features of the environment which would project to the chromatic properties of the corresponding point in the picture. For example, in a system of line drawing, where edgepoints project to line-points, every line-point on the picture plane will be associated with the feature edge in the corresponding point in the array". An illustration from Greenberg 2014 is given in (27): only visible edges are represented (as lines) on the picture. Further possible marking conventions are discussed in Greenberg 2014, but in the purely iconic case we will stick to edge marking because it suffices to provide a simple initial model of manual marking in signing space.

(27) Illustration of a simple marking convention: only visible edges are marked (Greenberg 2014)

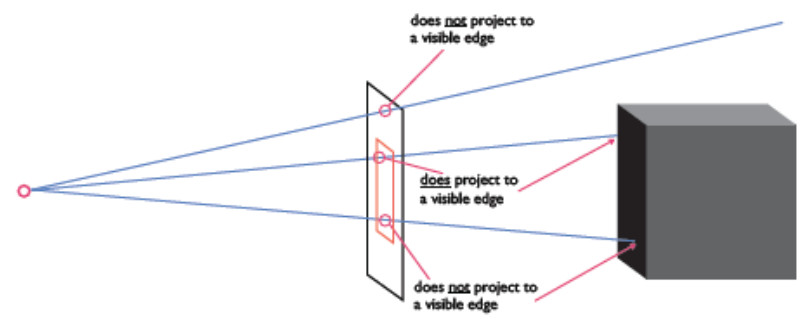

To be concrete, we will take the 2-dimensional space to be made of pixels, which may be filled or empty, as in (28) (one may think of 'filled' as 'black' and 'empty' as 'white', except that we be interested in signing space, where what matters is where parts of the hand appear).

\section{(28) Purely iconic marking rule}

Assuming a method of projection, mark a pixel as 'black' if and only if its projection line meets the edge of an object.

An alternative marking method is only semi-iconic, because the marking rule involves putting a linguistic symbol on a kind of map, following the statement in (29).

\section{(29) Mixed marking rule}

Assuming a method a projection, mark a pixel (which may be taken to be a large part of the 2dimensional space) with a given word $w$ just if and only if its projection line meets a certain type of object in the denotation of $w$.

To see the difference between these two methods, consider first the mixed marking rule in (29). We assume for greatest simplicity that the picture, here a vertical array in signing space, has only 9 pixels, and that projection is parallel. We restrict attention to the case discussed in the text: a $G$ of the manual alphabet, i.e. the shape $\$$, appears on a (large) pixel just in case a $G$ from the 2-dimensional contemporary drawing described can project onto it. We hasten to say that the representation on the left is a highly idealized representation of signing space. To give but one example, in unseparated repetitions the $\{$ shape appears continuously through space (this is what makes the iterations unseparated!), but a little forward movement of the hand indicates which parts of space are marked with $\$$ (see for instance the video associated with (8)).

(30) Example of the mixed marking rule for our ASL example in (8)

A highly simplified representation of signing space appears on the left, and the drawing which is discussed is represented on the right. 


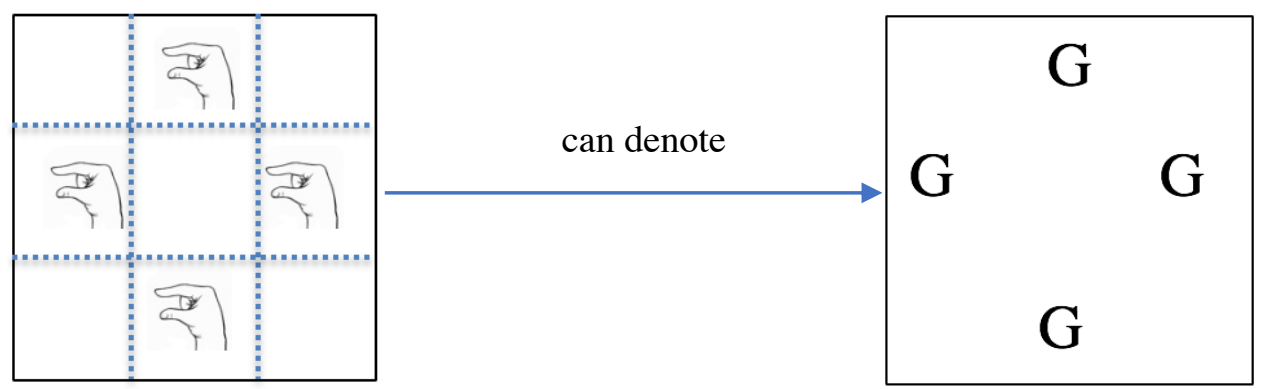

Importantly, the arrangement of the manual letters iconically represents the arrangement of their denotations, but the shape of the manual letters is entirely different from that of their denotations. This is because the marking rule is only semi-iconic: whether something gets represented in a pixel depends on the geometric projection, but what gets put in the pixel is a linguistic symbol.

Things are different in the purely iconic marking rule in (28), which is best illustrated with many more pixels, akin to a black-and-white photograph. Here the representation on the left can denote a drawing on the right just in case the latter genuinely resembles the former down to the details of the shapes involved. In the case discussed in the text, where a contemporary drawing is represented in signing space, the manual representation will roughly resemble what appears in the drawing.

(31) Example of the iconic marking rule for our ASL example in (12)

A highly simplified representation of signing space appears on the left, and the drawing which is discussed is represented on the right.

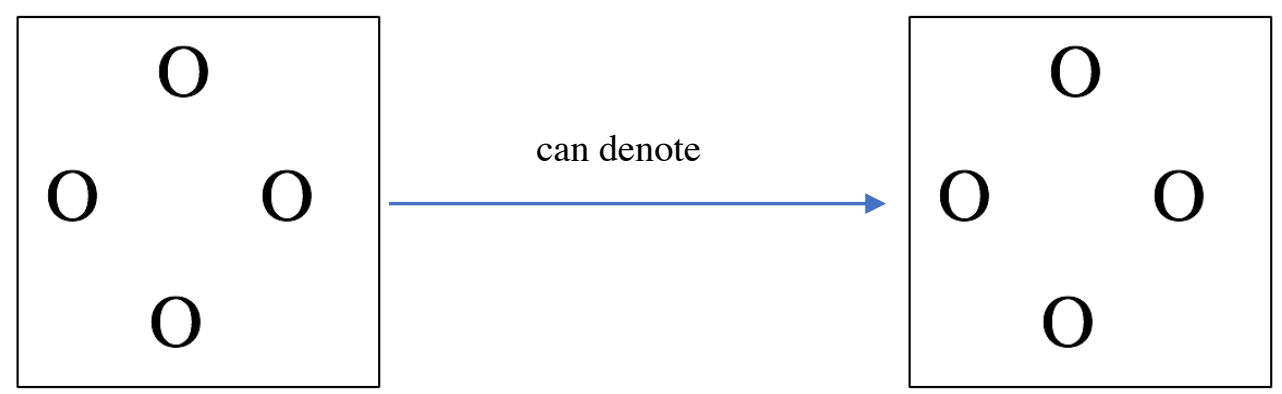

\subsection{Pictorial truth-of}

The interpretive rule for repetition-based plurals in (2) makes use of the notion of a noun 'resembling' an object. This would be relatively easy to make precise if we had a definition of what it means for part of a picture to represent an object. But our definition of truth for pictures doesn't quite yield this: it just defines what it means for a picture to be true of a viewpoint and of a world, not what it means for a picture part to be true of an object, a viewpoint and a world. Fortunately, this slightly different notion was briefly explored in Greenberg 2014 . We will call it pictorial truth-of to highlight the similarity with pictorial truth, but it is just the notion of a part of a picture representing an object. (The terminology we choose will be helpful because we will need to extend our analysis of truth for blurry pictures to truth-of.)

We follow Greenberg 2014 in taking as primitive a notion of grapheme, a "semantically significant" part of a picture, and in extending projection conditions a definition of truth-of. We do not seek to define more precisely what it means for an object d to project onto a grapheme $\mu$, but an illustration is given in (32).

(32) The picture on the left is true of the situation on the right, and the left-most shape denotes the topmost square (from Greenberg 2014) 

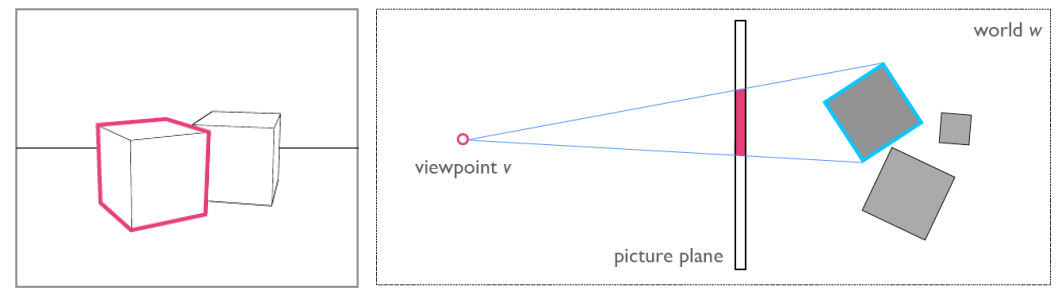

The definition of pictorial truth-of can then be given in (33). ${ }^{20}$

(33) Pictorial truth-of (inspired by Greenberg 2014)

A grapheme which is a part $\mu$ of a picture is true-of object $\mathrm{d}$ in world $\mathrm{w}$ relative to viewpoint $\mathrm{v}$ along the system of projection $\mathrm{S}$ iff $\mathrm{d}$ projects to $\mu$ from viewpoint $\mathrm{v}$ along $\mathrm{S}$, or in other words: $\mu$ is true-of $\mathrm{d}$ in world $\mathrm{w}$ from viewpoint $\mathrm{v}$ along system of projection $\mathrm{S}$ iff $\operatorname{projs}_{\mathrm{s}}(\mathrm{w}, \mathrm{v}, \mathrm{d})=\mu$.

Truth-of will be useful in our final analysis to make precise part (ii) of the semantics in (2), i.e. the requirement that the repeated noun should 'resemble' its denotation: resemblance will be cashed out in terms of 'truth-of'. But before we get there, we will develop most of the analysis in terms of truth (rather than truth-of) of pictures. ${ }^{21}$

\section{Blurry pictures}

A key distinction in ASL repetition-based plurals is whether the iterations are easy or hard to count. The former case can be handled by the simple picture semantics summarized in the preceding section. The latter case will be handled by a theory of blurry pictures, to which we now turn.

\subsection{Semantics through precisifications}

How should blurry pictures be interpreted? We develop an account within a precisification-based framework. Precisifications are commonly used in the analysis of vague terms in trivalent semantics, and they usually involve supervaluations (e.g. Fine 1975, and van Fraassen 1966 for a different use of supervaluations); we will see that refinements are needed in the case of pictures.

Take the schematic picture in (34)a, which has a blurry center and clear edges consisting of one cup each. We take the blurriness to consist in the fact that the center could correspond to one cup, yielding a total of three cups, as in (34)b, or to two cups, bringing the total to four, as in (34)c. From a precisification-based perspective, the basic intuition is that the blurry picture in (34)a can be made precise through (34)b as well as (34)c. When we consider these precisifications, we will assume that they include at least as many iterations as there are iterated expressions - hence at least three cups in (34).

Notations: $\mathrm{P}$ is the blurry picture in (34)a, $\mathrm{P}_{3}$ the precisification in $(34) \mathrm{b}$ and $\mathrm{P}_{4}$ the precisfication in (34)c.

\footnotetext{
${ }^{20}$ Greenberg 2014 proposes (in our terms) to define pictorial truth in terms of pictorial truth-of, but we will not be concerned with this reduction in the present piece.

${ }^{21}$ This paper was already written when Greenberg $2019 \mathrm{~b}$ appeared. It provides a semantics for tagging, a hybrid of symbols and pictures, used for instance in maps and in labelled pictures. We leave for future research an investigation of potential connections between the present piece and Greenberg's analysis of tagging.
} 
(34) Two precisifications (in b. and c.) of a blurry picture (in a. $)^{22}$

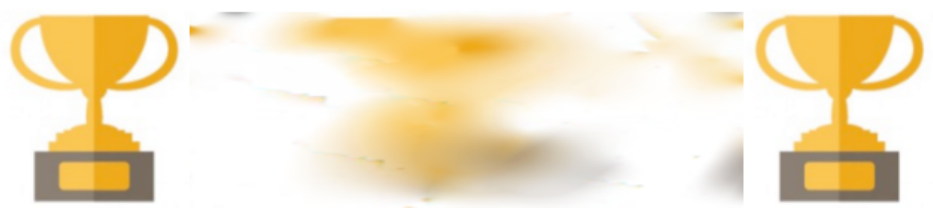

a.

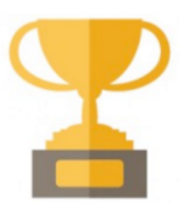

b.

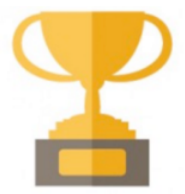

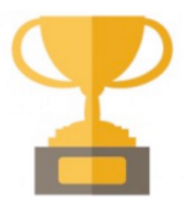

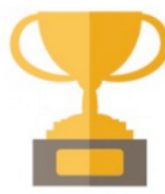

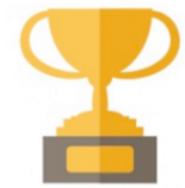

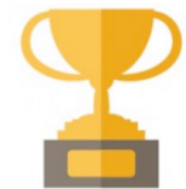

c.

Formally, then, we take a blurry picture to be associated with a set of precisifications, as stated in (35). In standard treatments of vagueness, precisifications are interpretations, but for simplicity we will take precisifications to be themselves non-blurry pictures. (It is clear that from a non-blurry picture one can infer an interpretation. We leave for future research a statement in terms of precise interpretations rather than precise pictures.)

\section{(35) Precisifications of a blurry picture}

A blurry picture $\mathrm{P}$ is associated with a set of precisifications $\Pi(\mathrm{P})=\left\{\mathrm{P}_{1}, \mathrm{P}_{2}, \ldots\right\}$, each of which is a picture which (i) has the same frame as $\mathrm{P}$, and (ii) is non-blurry.

The next step is to define the truth conditions of blurry pictures. There are two salient approaches to truth via precisifications. The most common view is based on strict truth, and it is associated with supervaluations. As stated in (36)a, a vague expression is strictly true just in case it is true on each of its precisifications. But we will have to make use of a more liberal notion, which is sometimes called tolerant truth (and is associated with 'subvaluations'). As stated in (32)b, a vague expression is tolerantly true just in case it is true on at least one of its precisifications (see for instance Varzi 1995, Hyde 1997, Cobreros et al. 2012).

\section{(36) Truth of vague expressions}

A vague expression is

a. strictly true iff it is true on each of its precisifications; strictly false iff it is false on each of its precisifications.

b. it is tolerantly true on at least one of its precisifications; tolerantly false iff it is false on at least one of its precisifications.

In view of the definition of falsity in (36)a, it is clear that an expression may be neither strictly true nor strictly false (in case it is true on some precisifications and false of others), which requires the use of truth gaps. And in view of (36)b, an expression may be both tolerantly true and tolerantly false (also in case it is true on some precisifications and false of others), which requires truth 'gluts'. Since the logics involved in the treatment of truth gluts are complex, we will use a simplified, bivalent notion of tolerant truth, defined in (37): on this analysis, an expression which is not tolerantly true is just false (see Cobreros et al. 2012 for a discussion of this approach and glut-based improvements of it).

\footnotetext{
${ }^{22}$ Here and throughout: pictures are modified from http://www.freepik.com/free-vector/flat-trophy- 
(37) Bivalent tolerant truth

A vague expression is tolerantly true on at least one of its precisifications; tolerantly false otherwise.

On this simplified notion, an expression is always true or false, and it behaves in this respect much like a modal statement (with 'tolerantly true' interpreted as 'possibly true', 'tolerantly false' as 'not possibly false').

\subsection{Using bivalent tolerant truth for blurry pictures}

Restricting attention to truth (rather than falsity) conditions, we can apply both notions of truth to blurry pictures, as in (38) (in a bivalent system, falsity conditions will immediately follow).

\section{(38) Truth of a blurry picture}

Relative to a world $\mathrm{w}$, a viewpoint $\mathrm{v}$ and a system of projection $\mathrm{S}$, a picture $\mathrm{P}$ associated with precisifications $\pi(\mathrm{P})=\left\{\mathrm{P}_{1}, \mathrm{P}_{2}, \ldots\right\}$ is

a. strictly true iff iff for each $\mathrm{P}_{\mathrm{i}}$ in $\pi(\mathrm{P})$, $\operatorname{projs}_{\mathrm{s}}(\mathrm{w}, \mathrm{v})=\mathrm{P}_{\mathrm{i}}$;

b. tolerantly true iff for at least one $\mathrm{P}_{\mathrm{i}}$ in $\pi(\mathrm{P}), \operatorname{proj}_{\mathrm{s}}(\mathrm{w}, \mathrm{v})=\mathrm{P}_{\mathrm{i}}$.

In standard linguistic applications, one often makes use of strict truth. But in the present case, this won't do: on the strict notion, a blurry picture will almost never ${ }^{23}$ be true of anything. The reason is that the precisifications provide incompatible information. Specifically, consider again the blurry picture in (34)a, assuming for concreteness that it is taken by a camera. The precisification in (34)b requires that there should be exactly three trophies in front of the camera, whereas the precisification in (34)c requires that there should be exactly four trophies in front of the camera. These are incompatible conditions, so the picture will never be strictly true.

Our pictorial case is different from standard instances of vagueness investigated in language. For bald, different precisifications establish different upper limits on the number of hairs that can be tolerated for the predicate to be true of the argument. If we call these precisfications bald $_{\leq n}$ for different values of the upper limit $\mathrm{n}$, different precisifications are compatible with each other; for instance a person (call him Peter) who has 0 hair will count as 'bald' on all the precisifications, and thus Peter is bald will in that case be strictly true.

There are two conclusions one could draw. One is that the correct notion of truth in the case at hand is that of tolerant truth, not strict truth (since the latter is essentially useless). An alternative would be to take the primitive notion to be the information entailed by a vague picture, rather than the worlds in which it is true. We will see that in a certain sense, both solutions boil down to the same thing. But let us first define the notion of tolerant truth for blurry pictures we will be using throughout. For simplicity, we keep it bivalent, leaving a multivalent account for future research.

\section{(39) Bivalent tolerant truth for a blurry picture}

Relative to a world $\mathrm{w}$, a viewpoint $\mathrm{v}$ and a system of projection $\mathrm{S}$, a picture $\mathrm{P}$ associated with precisifications $\pi(P)=\left\{P_{1}, P_{2}, \ldots\right\}$ is tolerantly true iff for at least one $P_{i}$ in $\pi(P), \operatorname{projs}_{s}(w, v)=P_{i}$; tolerantly false otherwise.

Since our initial semantic rule for repetition-based plurals in (2) involves "discourse referents made available by the iconic semantics" of the repeated noun, it will be useful to have a corresponding notion of truth-of, because the discourse referents in question will be analyzed as picture parts that denote certain objects. An initial definition is given in (40). We assume that, just like pictures, parts of pictures are associated with precisifications. Specifically, if a picture $\mathrm{P}$ has precisifications $\mathrm{P}_{1}, \mathrm{P}_{2}, \ldots$, then a part $\mu(\mathrm{P})$ of $\mathrm{P}$ has as possible precisifications the

\footnotetext{
${ }^{23}$ We write 'almost never' rather than 'never' because there could be special cases to consider. For instance, with marking rule we will adopt in Section 9.2, a picture may conceivably be 'silent' on what happens in part of the situation of evaluation. If so, a different picture may be true of the same situation relative to the very same viewpoint (e.g. if the second picture is identical to the first one except that it provides information on a component of the situation that the first one is silent on).
} 
corresponding parts $\mu\left(\mathrm{P}_{1}\right), \mu\left(\mathrm{P}_{2}\right), \ldots$ (the notion of a picture part could presumably be analyzed in terms of sets of pixels of an array, but we will take the notion as primitive here).

\section{(40) Bivalent tolerant truth-of for a blurry picture - simple case}

Relative to a world $\mathrm{w}$, a viewpoint $\mathrm{v}$ and a system of projection $\mathrm{S}$, a grapheme which is a part $\mu$ of a picture $\mathrm{P}$ associated with precisifications $\pi(\mathrm{P})=\left\{\mathrm{P}_{1}, \mathrm{P}_{2}, \ldots\right\}$ is tolerantly true-of an object $\mathrm{d}$ iff for at least one $\mathrm{P}_{\mathrm{i}}$ in $\pi(\mathrm{P})$, for the part $\mu_{\mathrm{i}}$ of $\mathrm{P}_{\mathrm{i}}$ that corresponds to $\mu$, $\operatorname{proj}_{\mathrm{s}}(\mathrm{w}, \mathrm{v}, \mathrm{d})=\mu_{\mathrm{i}}$. Otherwise, $\mu$ is tolerantly false of $d$.

The semantics in (2) involves the simultaneous creation of several discourse referents, which will be analyzed as several picture parts that each have a denotation. This requires a refinement to the definition in (40) to the case of tuples of discourse referents, as defined in (41).

(41) Bivalent tolerant truth-of for a blurry picture - general case

Relative to a world $\mathrm{w}$, a viewpoint $\mathrm{v}$ and a system of projection $\mathrm{S}$, a sequence of graphemes $\left\langle\mu^{1}, \ldots, \mu^{\mathrm{n}}\right\rangle$ which are part of a spicture $\mathrm{P}$ associated with precisifications $\pi(\mathrm{P})=\left\{\mathrm{P}_{1}, \mathrm{P}_{2}, \ldots\right\}$ is tolerantly true-of a sequence of objects $\left\langle\mathrm{d}_{1}, \ldots, \mathrm{d}_{\mathrm{n}}>\right.$ in world $\mathrm{w}$ iff for at least one $\mathrm{P}_{\mathrm{i}}$ in $\pi(\mathrm{P})$, for the parts $\mu_{\mathrm{i}}{ }^{1}, \ldots, \mu_{\mathrm{i}}^{\mathrm{n}}$ that correspond to $\mu^{1}, \ldots, \mu^{\mathrm{n}}$ respectively, $\operatorname{proj}_{\mathrm{s}}\left(\mathrm{w}, \mathrm{v}, \mathrm{d}_{1}\right)=\mu_{\mathrm{i}}{ }^{1}, \ldots, \operatorname{proj}_{\mathrm{s}}(\mathrm{w}, \mathrm{v}$, $\left.\mathrm{d}_{\mathrm{n}}\right)=\mu_{\mathrm{i}}{ }^{\mathrm{n}}$. Otherwise, $\left\langle\mu^{1}, \ldots, \mu^{\mathrm{n}}\right\rangle$ is tolerantly false of $\left\langle\mathrm{d}_{1}, \ldots, \mathrm{d}_{\mathrm{n}}\right\rangle$.

To see why this simultaneous evaluation of picture parts is needed, consider again the blurry picture in (34), with the rough division into parts displayed in (42). It makes sense to say that C1 and $\mathrm{C} 3$ can each denote a trophy (on the precisification in (34)c), and that $\mathrm{C} 2$ can denote a trophy as well (on the precisification in (34)b), but it won't do to say that $\mathrm{C} 1, \mathrm{C} 2$ and $\mathrm{C} 3$ can, together, denote 3 trophies, as this would bring the total to $5 .{ }^{24} \mathrm{It}$ is thus important to evaluate denotations of tuples simultaneously, as we do in (41).

left

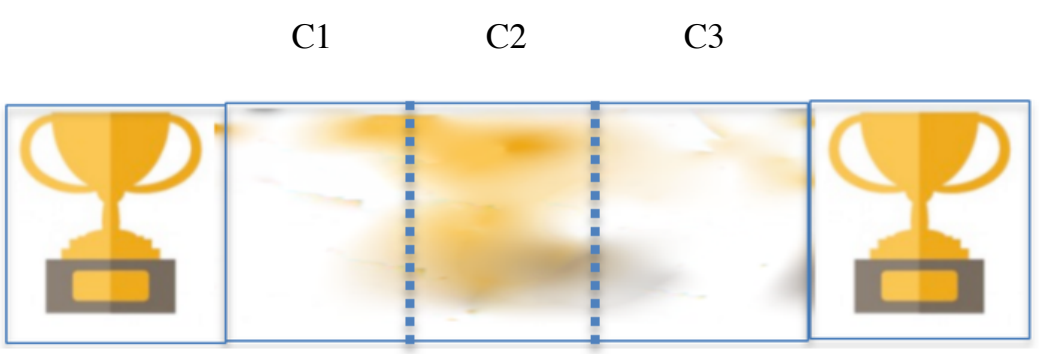

\subsection{Bivalent tolerant truth via entailments}

As we saw, strict truth as defined in (38)a is almost useless when it comes to blurry pictures. An alternative to using tolerant truth would be to take the primitive notion to be the information entailed by a vague picture, rather than the worlds in which it is true. To be concrete, consider again the blurry picture in (34)a. Intuitively, it does not entail that there are exactly three trophies in front of the camera, nor that there are exactly four trophies, but it does entail that there are at least three, and in fact it entails that there are exactly three or exactly four.

This notion could be cashed out in terms that are congenial to the strict notion of truth, as in (43). (43)a states the general case: a vague expression $E$ entails an expression $E^{\prime}$ just in case for each precisification $\pi$ of the interpretation, $\pi(E)$ entails $\pi\left(E^{\prime}\right)$. But we are interested in the more special case in (43)b, in which $\mathrm{E}$ is a picture and $\mathrm{E}^{\prime}$ is a standard, non-vague propositional expression.

\footnotetext{
${ }^{24}$ Technically, the problem is that we ought to ensure that the mereological sum $\mathrm{C} 1+\mathrm{C} 2+\mathrm{C} 3$ of the three relevant parts of the array can only denote 1 or 2 cups, whereas the sum of the denotations of $\mathrm{C} 1, \mathrm{C} 2$ and C 3 includes 3 cups.
} 
(43) Bivalent entailment for vague expressions

a. General case

A vague expression $E$ entails $E^{\prime}$ iff for each precisification $\pi, \pi(E)$ entails $\pi\left(E^{\prime}\right)$. (Otherwise, $E$ doesn't entail E'.)

b. Case in which $\mathrm{E}$ is a blurry picture and $\mathrm{E}^{\prime}$ is not vague

Consider a viewpoint $\mathrm{v}$ and a system of projection $\mathrm{S}$, let $\mathrm{P}$ be a picture with precisifications $\pi(\mathrm{P})$

and let $\mathrm{E}^{\prime}$ be a non-pictorial, non-vague expression (which may depend both on a world and a viewpoint of evaluation).

$P$ entails $E^{\prime}$ just in case for every world $w$, for every precisification $P_{i}$ in $\pi(P)$, if $\operatorname{proj}_{\mathrm{s}}(w, v)=P_{i}, E$

is true in $\mathrm{w}$ from viewpoint $\mathrm{v}$. (Otherwise, $\mathrm{P}$ doesn't entail $\mathrm{E}^{\prime}$.)

One could thus approach the truth of blurry pictures indirectly: we start by finding all the entailments $\mathrm{E}^{\prime}$ of a blurry picture $\mathrm{P}$, using the recipe in (43)b. Then we decree that $\mathrm{P}$ is true in a world $\mathrm{w}$ just in case each of its entailments is.

Concretely, consider again the blurry picture $P$ in (34)a, and let us restrict attention to four worlds $\mathrm{w}_{1}, \mathrm{w}_{2}, \mathrm{w}_{3}$ and $\mathrm{w}_{4}$, with respectively 1, 2, 3 and 4 trophies arranged 'in the right way' in front of the camera. It is clear that $P$ entails the proposition $\left\{\mathrm{w}_{3}, \mathrm{w}_{4}\right\}$ (or anything weaker), but nothing stronger. The indirect notion of truth specifies that $P$ is true in a world $\mathrm{w}$ just in case each of its entailments is, or equivalently, just in case its strongest entailment $\left\{\mathrm{w}_{3}, \mathrm{w}_{4}\right\}$ is. In the end, $P$ is true in $\mathrm{w}_{3}$ and $\mathrm{w}_{4}$. But this is also what the tolerant notion of truth would give us.

As we show in Appendix I, the two notions of truth - bivalent tolerant truth, and truth via entailments - yield the same result. This gives us a modicum of confidence that this notion of truth is rather natural in the case at hand. It remains an overly simple notion because it obliterates trivalence and genuine vagueness, and thus refinements should be explored in the future. But we henceforth use this notion of bivalent tolerant truth in our analysis.

\subsection{Deriving 'at least' readings}

With a theory of bivalent tolerant truth for blurry pictures in hand, we can derive the existence of 'at least' readings for unpunctuated repetitions, but we need one additional hypothesis: instead of considering just two precisifications, as in our initial example in (34), we must consider infinitely many. Specifically, for $\mathrm{n}$ unpunctuated iterations, we will consider precisifications with $\mathrm{i}$ iterations for each $\mathrm{i} \geq \mathrm{n}$, as is stated in (44).

\section{(44) Precisifications to derive 'at least' readings}

If $P$ involves $n$ unpunctuated (i.e. hard to count) iterations of an expression $E$ (which may be iconic or conventional), then $\pi(\mathrm{P})=\left\{\mathrm{P}_{\mathrm{i}}: \mathrm{i} \geq \mathrm{n}\right\}$, where $\mathrm{P}_{\mathrm{i}}$ is a precisification with exactly $\mathrm{i}$ iterations of $E$.

To illustrate how (39) and (44) can be combined, consider (8)b. It involves 4 unseparated fast iterations of the manual letter $G$. The iterations are particularly hard to count, and are thus a clear example of an unpunctuated repetition, which we write as $G$-rep $4_{\text {circular }}$. If we write as $G^{i}$ the precisifications of $G$-rep4 $4_{\text {circular }}$ that involve exactly i iterations of $G$, arranged in the same (circular) shape as $G$-rep $4_{\text {circular }}$, we can define the set of precisifications specified by (44):

(45) Precisifications of $G$-rep4 $4_{\text {circular }}$ (as in (8)b)

$\pi\left(\mathrm{G}-\right.$-rep $\left.4_{\text {circular }}\right)=\left\{\mathrm{G}^{\mathrm{i}}: \mathrm{i} \geq \mathrm{n}\right\}$, where $\mathrm{G}_{\mathrm{i}}$ is a precisification with exactly i iterations of $G$-rep $4_{\text {circular }}$.

We can now say under what conditions G-rep4 $4_{\text {circular }}$ is (bivalently tolerantly) true. Assuming the mixed marking rule in (29), each $G^{i}$ will come with a requirement that there should be exactly i G's arranged in a circular fashion. The bivalent tolerant truth conditions are given in (46).

(46) Tolerant truth of $G$-rep $4_{\text {circular }}$ with the precisifications in (45)

Relative to a world w, a viewpoint v and a system of projection S, G-rep $4_{\text {circular }}$ is tolerantly true iff for at least one $\mathrm{G}^{\mathrm{i}}$ in $\pi\left(\mathrm{G}-\mathrm{rep} 4_{\text {circular }}\right)$, $\operatorname{proj}_{\mathrm{s}}(\mathrm{w}, \mathrm{v})=\mathrm{G}^{\mathrm{i}}$,

iff (roughly) for some $\mathrm{i} \geq 4$, there are exactly i G's arranged in a circular fashion.

Otherwise, G-rep $4_{\text {circular }}$ is tolerantly false. 


\subsection{The Edge Effect revisited}

Our approach based on precisifications makes it possible to revisit a constraint on anaphora noted in Schlenker and Lamberton 2019. As argued in that paper, punctuated repetitions introduce by default one discourse referent per iteration, but arguably unpunctuated repetitions only introduce discourse referents at the edges (they call this the 'Edge Effect'). The main effect is illustrated in (47): a possessive pronoun indexing the right-most iteration of the unpunctuated repetition of $T R O P H Y$ is acceptable, and refers to the right-most trophy; a possessive pronoun indexing the intermediate iteration is degraded. When the unpunctuated repetition is replaced with a punctuated repetition, any pattern of indexing is available.

(47) HERE HAVE TROPHY-rep3 $3_{\text {horizontal. }}$

'Here there are several trophies arranged in a row.'

a. ${ }^{7}$ [no additional sentence]

b. ${ }^{7}$ POSS-right SHAPE STRANGE.

'The right-most trophy has a weird shape.'

$\Rightarrow$ the right-most trophy has a weird shape

c. ${ }^{5}$ POSS-middle SHAPE STRANGE.

'The intermediate trophy has a weird shape.'

$\Rightarrow>$ the intermediate trophy has a weird shape

(ASL, 33, 0592; 3 judgments; Schlenker and Lamberton 2019)

In triangular representations, each vertex of the triangle introduces a separate discourse reference (hence the 'Edge' effect really pertains to vertices, but the latter terminology is a bit too technical to be optimally useful).

Schlenker and Lamberton 2019 suggest that the source of the contrast is that in unpunctuated repetitions the intermediate iteration (out of 3 ) is somehow blurry, as illustrated in (48), but this earlier paper does not offer a formal account (although it does draw a connection with theories of iconically generated loci in arc-based plural pronouns).

(48) Successful pointing (on the left) and unsuccesful pointing (in the middle)

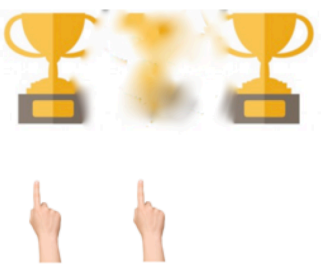

We propose that a constraint on pointing is that the outer frontiers of the area pointed to should be clear rather than blurry. This can be cashed out in terms of precisifications. In a nutshell, we will assume that pointing is always towards a part $\mu$ of a picture which (i) has a natural shape, and also (ii) has clear edges, i.e. parts that are edges in all precisifications of the blurry picture. This is made more precise in (49). Importantly, we leave open several notions, such as what a 'natural shape' is, and what it means for a point of a representation to be 'between' points of an edge. This is because these cognitive notions are likely complex, and here we are just interested in understanding how they interact with pictorial vagueness.

\section{(49) Availability for pointing of picture parts}

a. A part of a picture is available for pointing only if (i) it has a natural shape, (ii) it is delimited by clear edges.

b. A part $\mu$ of a (possibly blurry) picture $\mathrm{P}$ is delimited by clear edges just in case there is a set $\varepsilon$ of pixels of $\mu$ such that for each precisification $\mathrm{P}_{\mathrm{i}}$ in $\pi(\mathrm{P})$, in $\mathrm{P}_{\mathrm{i}}$ : 
each point of $\varepsilon$ is marked;

each point of $\mu$ which is marked is either within $\varepsilon$, or included between two points of $\varepsilon$.

The intuition we develop is that leftward pointing in (48) is acceptable because the outer edges of the left-most trophy are sharp. Pointing towards the center is less acceptable because the outer edges of the intermediate trophy are not sharp. Still, we wouldn't want to prohibit all pointing towards a representation that has a blurry component, as one can certainly point towards the entire set of trophies in (48) (the same observation extends to sign language: it is clear that an unpunctuated repetition as a whole can introduce a discourse referent, even though its blurry central component doesn't $\mathrm{t}^{25}$ ). We could try to develop the analysis in semantic terms, by talking about the edges of the denoted groups, but at this point we find it simpler to talk about the edges of the representation rather than of its denotations (we leave a comparison for future research).

To apply the theory, we consider once again the blurry picture in (34)a with the two precisifications in (34)b,c, and consider in turn different patterns of pointing involving the three regions depicted in (50).

(50) Three parts of the blurry picture in (34)a

\begin{tabular}{|llll} 
& center & right \\
\hline & & \\
\hline
\end{tabular}

It is clear that left pointing is acceptable because the left-most part of the picture is entirely sharp: all precisifications agree on the left-most part, and as a result the outer edges of the left-most trophy are sharp. The same conclusion applies to the outer edges of the right-most trophy. Pointing towards the center won't work in the same way, however. The reason is that no set of pixels counts (on any reasonable definition) as a sharp edge of the intermediate trophy or trophies. This is so because the outer edges of the intermediate component in (51)a (continuous line) are just different from the outer edges of the intermediate component in (51)b (also represented with a continuous line), so there is no way these edges could be sharp. Furthermore, we could use as sharp edges the innermost edges of the left-most and right-most trophies (hatched lines), but the resulting area certainly doesn't correspond to a natural shape (it would be made of the intermediate trophy/trophies with the innermost edges of the extreme trophies). Finally, nothing in this account prohibits pointing towards the entire set of trophies: although their center is blurry, their edges are sharp, and thus the condition for pointing in (49) is satisfied. (In the triangular case, we would

\footnotetext{
${ }^{25}$ Still, the analogy with the behavior of a whole unpunctuated repetition in ASL shouldn't be exaggerated. It might be through grammatical means that a plural discourse referent is introduced by the entire set of repetitions. By contrast, iconic means might be needed to introduce singular discourse referents at the edges. This possible claim was tested by Schlenker and Lamberton 2019 with a paradigm that included (i). The idea was that plural reference to the entire set of sentences described by (i) could be effected grammatically, while singular reference to an individual sentence should require an iconic interpretation and an edge. As noted in that earlier paper, "the first sentence evaluated on its own only provides weak iconic information. Adding the second clause with a right-edge possessive pronoun strengthens the iconic inference in (...) [(i)a)], but the effects are not entirely clear".

(i) YESTERDAY YOU WRITE MEANING VAGUE.

'Yesterday you wrote sentences. The meaning of the right-most sentence/of all thesentences was vague.'

a. ${ }^{6.3}=$ SENTENCE-rep 3 horizontal $\quad \ldots=$ POSS-right

$3 / 3$ judgments: the sentences are more likely to be in a row when the 2 nd clause is added

b. ${ }^{7}=$ SENTENCE-rep3 $3_{\text {horizontal }} \quad \ldots=$ POSS - arc

$1 / 3$ judgment: the sentences are more likely to be in a row when the 2 nd clause is added ( $\underline{\text { ASL, 34, 2256 }} \mathrm{c}, \mathrm{d} ; 3$ judgments)
} 
need to say that the three sharp vertices are enough to satisfy this condition, which makes intuitive sense.)

(51) Precisifications of the picture in (50)

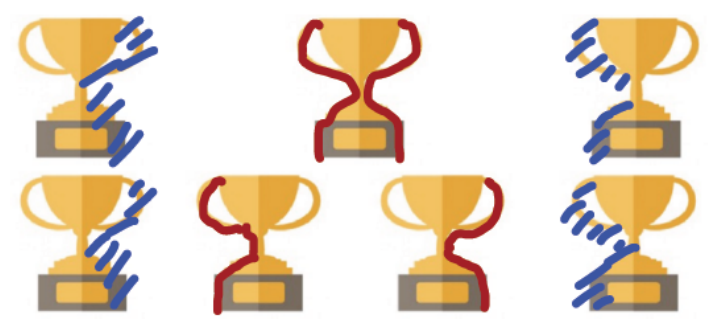

Both the empirical and the theoretical side of the Edge Effect will have to be refined in future research. There are certainly alternative ways to develop the analysis. But we hope to have shown that an approach based on precisifications can help make potential theories sharper and possibly more explanatory.

\subsection{Roles of vagueness}

At this point, we have seen that blurry pictures can be analyzed by way of a precisification-based semantics. A notion of strict truth is of little interest because the various precisifications of a blurry picture usually make incompatible demands: one may require that there are exactly three trophies in front of the camera, while the other may require that there are exactly four, and no world can satisfy both. We must thus appeal to a tolerant notion of truth, one on which a blurry picture is (tolerantly) true just in case one of its precisifications is true. The bivalent notion we used obliterates trivalence, since a blurry picture is either tolerantly true or tolerantly false, never indeterminate. We saw, however, that the same notion can be motivated in a different way, by starting from the propositional entailments of a picture. An additional benefit of the precisification-based approach is to offer tools to state possible hypotheses about the source of the Edge Effect described in Schlenker and Lamberton 2019. While we have not attempted to give the final word on this issue, we used a notion of 'sharp edges' that might provide the beginning of a solution.

\section{Revisiting the truth conditions of ASL repetition-based plurals}

While we have provided an account of the truth of iconic representations involving repetitions, we have not explained how our initial definition of truth for sentences with repetition-based plurals should be amended in light of this analysis. Two components of the original definition in (2), repeated in (52), should be taken care of: the part appealing to an iconic representation in (52)(ii), and that pertaining to the discourse referents made available by the iconic semantics of the repeated expression in (52)(iii).

(52) For $N$-iter ${ }_{X}=N$-rep $p_{X}$ or $[N N N]_{X}$ or $N$-cont , if $P$ is a propositional expression $\left[\left[N-i t e r{ }_{X} \mathrm{P}\right]\right]^{\mathrm{c}, s}=$ true iff for some group $\mathrm{d}$,

(i) $[\mathrm{N}]]^{\mathrm{c} s[\mathrm{X} \rightarrow \mathrm{d}]}(\mathrm{d})=$ true, and

(ii) $N$-iter iconically represents d given c, and

(iii) $[\mathrm{P}]]^{\mathrm{c}, \mathrm{s}\left[\mathrm{X} \rightarrow \mathrm{d}, \mathrm{x}_{1} \rightarrow \mathrm{d}_{1}, \ldots, \mathrm{x}_{\mathrm{n}} \rightarrow \mathrm{d}_{\mathrm{n}}\right]}=$ true, where $x_{1}, \ldots, x_{n}$ are discourse referents made available by the iconic semantics of $N$-iter, and $x_{1}, \ldots, x_{n}$ respectively denote $\mathrm{d}_{1}, \ldots, \mathrm{d}_{\mathrm{n}}$ (which are parts of $\mathrm{d}$ ).

We propose to make this interpretive rule more precise as follows (further refinements will have to be explored in the future).

(53) For $N$-iter ${ }_{X}=N-$ rep $_{X}$ or $[N N N]_{X}$ or $N$-cont , $_{X}$, if $P$ is a propositional expression, $[[\mathrm{N} \text {-iter } \mathrm{P} \mathrm{P}]]^{\mathrm{c}, \mathrm{s}}=$ true iff for some viewpoint $\mathrm{v}$ and for system of projection $\mathrm{S}$ made available by $\mathrm{c}$, for some group $\mathrm{d}$, 
(i) $[\mathbb{N}]^{c}, s[X \rightarrow d](d)=$ true, and

(ii) $N$-iter is tolerantly true of $\mathrm{d}$, and

(iii) $[\mathbb{P}]^{c, s\left[X \rightarrow d, x_{1} \rightarrow d_{1}, \ldots, x_{n} \rightarrow d_{n}\right]}=$ true, where $x_{l}, \ldots, x_{n}$ is a maximal set ${ }^{26}$ of parts of $N$-iter which are available for pointing in $N$-iter and $\left\langle\mathrm{x}_{1}, \ldots, \mathrm{x}_{\mathrm{n}}\right\rangle$ is tolerantly true of of $\left\langle\mathrm{d}_{1}, \ldots, \mathrm{d}_{\mathrm{n}}\right\rangle$ and $\mathrm{d}_{1}, \ldots, \mathrm{d}_{\mathrm{n}}$ are parts of $d$.

In the case, discussed in Schlenker and Lamberton 2019, in which $N$ is a word (rather than an iconic representation), the system of projection $\mathrm{S}$ will have to involve the kind of mixed marking rule exemplified in (29). ${ }^{27}$

To illustrate the new statement in (53)(ii), consider first a derivation of the truth conditions of (8)a, repeated as (55)a, and analyzed with the simplified Logical Form in (55)b. Because the repetitions are arranged in a circular fashion, there are no edges and we assume that the rule in (53)(iii) has no effect: no individual discourse referents are introduced. In line with our earlier discussions, we take the precisifications of $G$-unsep $4_{\text {fast-circular }}$ to be all the clear (parts of) pictures with i iterations of $G$ arranged in a circular fashion, for $i \geq 4$; this is defined in (54).

(54) Precisifications of G-unsep $4_{\text {fast-circular }}$

Writing $G^{i}$ for i iterations of $G$ arranged in the same circulare shape as in $G$-unsep $4_{\text {fast-circular }}$, the precisifications of $G$-unsep $4_{\text {fast-circular }}$ are given by:

$\pi\left(\mathrm{G}-\right.$ unsep $\left._{\text {fast-circular }}\right)=\{\mathrm{G}: \mathrm{i} \geq 4\}$

(55) a. POSS-1 DRAWING G-unsep4 $4_{\text {fast-circular }}$

'My drawing contains at least 4 or 5 G's (arranged in a circular fashion).'

a'. G-unsep $4_{\text {fast-circular } x}$ [my-drawing has $\left.\mathrm{X}\right]$

b. $\left[\left[\left(\mathrm{a}^{\prime}\right)\right]\right]^{\mathrm{c}, \mathrm{s}}=$ true iff for some viewpoint $\mathrm{v}$ and for system of projection $\mathrm{S}$ made available by $\mathrm{c}$, for some group $\mathrm{d}$,

(i) $[\mathrm{G}]]^{\mathrm{c}, \mathrm{s}[\mathrm{X} \rightarrow \mathrm{d}]}(\mathrm{d})=$ true, i.e. $\mathrm{d}$ is a groupe of $\mathrm{G}^{\prime} \mathrm{s}$, and

(ii) G-unsep $4_{\text {fast }}$ is tolerantly true of $\mathrm{d}$, and

(iii) $[\text { my-drawing has } \mathrm{X}]^{\mathrm{c}, \mathrm{s}[\mathrm{X} \rightarrow \mathrm{d}]}=$ true, i.e. my drawing has $\mathrm{d}$.

In view of (54), (ii) is equivalent to: for some $\mathrm{i} \geq 4, \operatorname{projs}_{s}(w, v, d)=G^{i}$, i.e. (roughly) $d$ is made of at least 4 G's arranged as a circle.

Let us now consider the case of 3 unpunctuated iterations, as in (47)a, repeated as (57)a, with the schematic Logical Form in (57)a', for a complex predicate $P$ (which will contain the variable $X$ ). The precisifications of TROPHY-rep $3_{\text {horizontal }}$ are given in (57). The derivation of the truth conditions now involves the creation of new singular discourse referents, which could have an effect if $P$ contained singular pronouns.

(56) Precisifications of TROPHY-rep3 $3_{\text {horizontal }}$

Writing TROPHY horizontal for i iterations of TROPHY arranged in the same horizontal shape as in

TROPHY-rep $3_{\text {horizontal }}$, the precisifications of TROPHY-rep $3_{\text {horizontal }}$ are given by:

$\pi\left(\right.$ TROPHY-rep $\left.3_{\text {horizontal }}\right)=\left\{\right.$ TROPHY $\left.^{\mathrm{i}}{ }_{\text {horizontal }}: \mathrm{i} \geq 3\right\}$

(57) a. HERE HAVE TROPHY-rep3 $3_{\text {horizontal. }}$

'Here there are several trophies arranged in a row.'

a'. TROPHY-rep3 $3_{\text {horizontal } x} P$

b. $\left[\left[\left(a^{\prime}\right)\right]^{c, s}=\right.$ true iff

${ }^{26} \mathrm{We}$ add to (52)(iii) a maximality condition to make sure that all parts that are available for pointing are taken into account.

${ }^{27}$ Note that the mixed marking rule yields a partial redundancy between conditions (53)(i) and (53)(ii). Take for instance a circular punctuated repetition of manual G's, i.e. ₹, as in (40). By (53)(i), the denoted group $d$ will have to satisfy the condition that $\left.\left[[]^{\mathrm{T}}\right]\right]^{\mathrm{c}, \mathrm{s}[\mathrm{X} \rightarrow \mathrm{d}]}(\mathrm{d})=$ true, or in other words: $\mathrm{d}$ is a (singular or plural) group of G's. By (53)(ii), the is 's in signing space have to pictorially represent G's, hence the same result. Future research could seek to eliminate the redundancy by doing without condition (53)(i), but one point should be kept in mind: (53)(i) makes reference to a context and an assignment function, whereas (53)(ii) doesn't. Thus if a repeated noun has a context- or assignment-sensitive component, getting rid of (53)(i) might create problems. 
(i) $\left[[T R O P H Y]^{c, s[X \rightarrow d]}(d)=\right.$ true, i.e. $d$ is a group of trophies, and

(ii) $T R O P H Y$-rep $3_{\text {horizontal }}$ is tolerantly true of d, i.e. for some $\mathrm{i} \geq 3, \operatorname{projs}_{\mathrm{s}}(\mathrm{w}, \mathrm{v}, \mathrm{d})=$

TROPHYi ${ }_{\text {horizontal }}$, i.e. (roughly) $d$ is made of at least 3 trophies arranged horizontally,

(iii) $[\mathrm{PP}]^{\mathrm{c}, s\left[\mathrm{X} \rightarrow \mathrm{d}, \mathrm{x}_{1} \rightarrow \mathrm{d}_{1}, \ldots, x_{\mathrm{n}} \rightarrow \mathrm{d}_{\mathrm{n}}\right]}=$ true, where $x_{1}, \ldots, x_{n}$ is a maximal set of parts that are available for pointing in TROPHY-rep $3_{\text {horizontal }}$

and $\left\langle\mathrm{x}_{1}, \ldots, \mathrm{x}_{\mathrm{n}}\right\rangle$ is tolerantly true of of $\left\langle\mathrm{d}_{1}, \ldots, \mathrm{d}_{\mathrm{n}}>\right.$ relative to $\mathrm{v}$ and $\mathrm{S}$ and $\mathrm{d}_{1}, \ldots, \mathrm{d}_{\mathrm{n}}$ are parts of $\mathrm{d}$.

By the same reasoning as in (50), only the two extreme iterations have clear edges and are thus available for pointing, which means that (iii) reduces to (iii'):

(iii') $[[\mathrm{P}]]^{\mathrm{c}, s\left[\mathrm{X} \rightarrow \mathrm{d}, \mathrm{x}_{1} \rightarrow \mathrm{d}_{1}, \mathrm{x}_{2} \rightarrow \mathrm{d}_{2}\right]}=$ true, where $x_{1}$ and $x_{2}$ are the extreme iterations of TROPHY-rep $3_{\text {horizontal }}$ and $\left\langle\mathrm{x}_{1}, \mathrm{x}_{2}\right\rangle$ is tolerantly true of of $\left\langle\mathrm{d}_{1}, \mathrm{~d}_{2}\right\rangle$ and $\mathrm{d}_{1}$ and $\mathrm{d}_{2}$ are parts of $\mathrm{d}$.

Here we have followed Schlenker and Lamberton 2019 in giving a static analysis of discourse referents, but to handle anaphoric examples like (47), we would need to add to the analysis a dynamic account of discourse referent transmission across sentences, as already argued for ASL in Schlenker 2011. Since the dynamification of the present system is a separate (and rather standard, if complex) issue, we do not discuss it further in this piece.

\section{Pragmatic exploitation}

\subsection{Problems}

Our analysis based on vagueness and tolerant truth derives the main data but suffers from a drawback: when evaluating the number of iterations produced in cases of unpunctuated repetitions, it just isn't very plausible that there could be an arbitrary number of them. Take three blurry iterations, as in (34)a. Vagueness yields uncertainty about their number: there could be 3, or 4 . In the case of unpunctuated repetitions that are realized as fast unseparated iterations, as in (8)b, there might be greater uncertainty, e.g. 3, 4, 5 or 6 iterations. This wouldn't yield an 'at least' reading, but rather an 'approximately' reading. In other words, there is a discrepancy between the number iterations that one infers on purely iconic grounds (uncertainty between 3, 4, 5 and 6, for instance), and what the theory requires in order to derive an 'at least' reading (uncertainty among all numbers greater than or equal to 3). A more plausible view would be that there are various possible sets of precisifications, and that the one that yields the 'at least' reading is possible but extremely unlikely, while there are several others.

Another potential challenge is that our current analysis just doesn't yield any 'approximately' readings. But some are arguably instantiated. Take (17)b, the unseparated fast repetition of a manual $(=\mathrm{G})$ which gradually opens from one iteration to the next (hence a preferentially iconic reading, pertaining to the very shape displayed). One of the dominant inferences is that the relevant drawing has approximately four of these shapes (rather than exactly four). Now this fact could be analyzed by way of a standard implicature: one would start from a reading akin to 'at least four or so' (where or so represents a vague component that we don't derive with bivalent tolerant truth). This would then compete with five unseparated fast iterations of the same kind, corresponding to a quantity of 'at least five or so', and by negating this more informative alternative, we might conceivably get something akin to 'approximately four'.

But this reasoning won't work as well in (8)c, which involves the unseparated slow repetition of the manual The problem is that this expression doesn't seem to have an 'at least' reading. The dominant reading is an 'exactly' reading, but there also seems to be a weaker 'approximately' reading. To derive it, we need to use precisifications, but we shouldn't use the infinite set of precisifications that gives rise to the 'at least' reading. In this case, the small number of precisifications we considered in our initial formal example in (34)b would seem to do the job rather well. 
In sum, we have two main challenges to address: (i) we must explain why the 'at least' reading is so salient despite the fact that, on iconic grounds, it should be extremely unlikely; (ii) we must explain why there is an 'approximately' reading alongside the 'at least' reading, one that is less salient than the 'at least' reading despite being more plausible on iconic grounds.

\subsection{A 'bare bones' model}

We propose to start addressing the problem by making use of strategic reasoning.$^{28}$ We will follow the spirit but not the letter of the Rational Speech Act (RSA) model surveyed in Goodman and Frank 2016, Franke and Jäger 2016, and Bergen et al. 2016: our pragmatic analysis is tailored to the very special assumptions needed for our semantic theory of iconic vagueness in repetitionbased plurals. Furthermore, since there are already many moving parts in our analysis, we make the simplest possible assumptions needed to display the strategic effect we are interested in, leaving it for future research to develop more fine-grained and possibly more standard models. One simplifying assumption is that we take the agents to be perfectly rational, whereas this is only a special and rather unusual case in the RSA (that in which the 'temperature parameter' is set to infinity); this will allow us to display the main effects with virtually no computations. Another simplifying assumption is that we disregard the cost of different signals in our analysis, because the main desired effect can be displayed without it (we come back to its importance in Appendix II).

Our pragmatic analysis will be developed as a communication game with the following general properties. We will restrict attention to 3 or 4 punctuated or unpunctuated repetitions of the manual 7 , written as before as: $G G G, G G G G, G$-rep3, G-rep4. The speaker seeks to transmit a meaning among the following: exactly $\mathrm{n}$ G's (written as $=n$ ), at least $\mathrm{n}$ G's (written as $\geq n$ ), and approximately $n \mathrm{G}^{\prime}$ s (written as $\pm n$, and identified to ' $n$ or $n+1$ '). Thus the information transmitted is not about what the speaker knows about the world, but about the meaning that she has in mind (for a particular constituent, which may be embedded as in several of our examples, e.g. in Section 4). Punctuated repetitions have an unambiguous literal meaning, but unpunctuated repetitions, due to their blurriness, are ambiguous among several 'approximately' and 'at least readings'.

At an initial level, a naive addressee $\mathrm{A}_{0}$ receives one of these four possible signals, and seeks to assign a meaning to it. For punctuated repetitions, this is easy as they have just one possible meaning: $G G G$ means 'exactly 3 G's' (i.e. $=3$ ), while $G G G G$ means 'exactly 4 G's' (i.e. =4). But the blurriness of unpunctuated repetitions makes them ambiguous, as a blurry picture may be associated with different sets of precisifications. Given a (blurry) signal, a set of precisifications fully determines its meaning (in view of our analysis in terms of bivalent tolerant truth), and different precisifications yield different meanings, so the probability of an interpretation is fully given by the probability of the corresponding set of precisifications. The probabilities of different sets of precisifications are given by perceptual considerations: on perceptual grounds, 3 unpunctuated iterations are more likely to be associated with a ' 3 or 4' interpretation (meaning that perceptually, it's unclear whether there are 3 or 4 G's depicted) than with an 'at least 3' interpretation (meaning that it's unclear whether there are 3, 4, 5, 6, ... G's depicted).

At the next level, we will consider a strategic speaker $S_{1}$ who takes into account the behavior of $\mathrm{A}_{0}$ : starting from a target meaning, $\mathrm{S}_{1}$ will pick the form that maximizes the chance that $\mathrm{A}_{0}$ recovers it. For 'exactly' readings, punctuated repetitions are an obvious choice because they are unambiguous. For 'approximately' and 'at least' readings, $S_{1}$ has no choice but to pick unpunctuated repetitions, which are ambiguous. How often $S_{1}$ picks an unpunctuated repetition to express an 'at least' vs. an 'approximately' reading will depend on $S_{1}$ 's general propensity to choose one meaning or the other.

\footnotetext{
${ }^{28}$ Special thanks to B. Spector (p.c.) for several helpful discussions, and to an anonymous reviewer for urging that we clarify the initial presentation of the model.
} 
At the next and final level, the strategic addressee $A_{1}$ takes into account $S_{1}$ 's behavior and interprets an unpunctuated repetition with an 'at least' or an 'approximately' reading in view of assumptions about $S_{1}$ 's propensity to choose one meaning or the other. The key at this point is that the perceptual implausibility of the 'at least' reading, which affected $\mathrm{A}_{0}$, does not affect $\mathrm{A}_{1}$ any longer, because $\mathrm{A}_{1}$ realizes that if $S_{1}$ wants to express an 'at least' reading, an unpunctuated repetition is her best choice: no matter how iconically implausible the 'at least' reading was on iconic grounds, this won't play a role at this stage. At this point, then, the probability of the two readings ('at least' vs. 'approximately') is not determined by perceptual considerations any more, but by assumptions about how often $S_{1}$ may want to express one meaning or the other.

\subsection{Model assumptions}

We recapitulate in (58) and (59) the possible messages and meanings we will consider.

(58) Messages

G G G

G-rep3

G G G G

G-rep4

(59) Meanings the messages may have

$\begin{array}{llll}=3 & =4 & =5 & \\ \pm 3 & \pm 4 & \pm 5 & \\ \geq 3 & \geq 4 & \geq 5 & \geq 6\end{array}$

The literal meanings of punctuated repetitions are certain, as in (60).

(60) Meanings of punctuated repetitions

$\operatorname{Pr}($ meaning is $=3$ I signal is $\mathrm{G} \mathrm{GG})=1$

$\operatorname{Pr}($ meaning is $=4$ I signal is $\mathrm{G} \mathrm{G} \mathrm{G} \mathrm{G})=1$

For unpunctuated repetitions, different sets of precisifications come with different probabilities. We have chosen those in (61) to highlight the fact that 3 unpunctuated iterations are more likely to have an 'exactly 3 ' than an 'exactly 4 ' reading, that an 'approximately 3 ' reading is possible as well and more likely than an 'approximately 4 ' reading, and that an 'at least 3 ' reading is rather unlikely (while an 'at least 4' reading is even less likely).

(61) Possible meanings of G-rep 3

\begin{tabular}{|l|l|l|}
\hline Precisifications & Corresponding meaning & $\begin{array}{l}\text { Pr(meaning is ... I signal } \\
\text { is G-rep })_{3}\end{array}$ \\
\hline$\left\{\mathrm{G}_{3}\right\}$ & $=3$ & $1 / 4$ \\
\hline$\left\{\mathrm{G}_{4}\right\}$ & $=4$ & $1 / 8$ \\
\hline$\left\{\mathrm{G}_{3}, \mathrm{G}_{4}\right\}$ & \pm 3 & $1 / 4$ \\
\hline$\left\{\mathrm{G}_{4}, \mathrm{G}_{5}\right\}$ & \pm 4 & $1 / 8$ \\
\hline$\left\{\mathrm{G}_{\mathrm{k}}: \mathrm{k} \geq 3\right\}$ & $\geq 3$ & $1 / 8$ \\
\hline$\left\{\mathrm{G}_{\mathrm{k}}: \mathrm{k} \geq 4\right\}$ & $\geq 4$ & $1 / 16$ \\
\hline$\left\{\mathrm{G}_{\mathrm{k}}: \mathrm{k} \geq 5\right\}$ & $\geq 5$ & $1 / 16$ \\
\hline
\end{tabular}

Possible meanings for $G$-rep 4 are similar, adding everywhere an increment of 1 .

(62) Possible meanings of $\mathrm{G}_{\text {-rep }}$

\begin{tabular}{|l|l|l|}
\hline Precisifications & Corresponding meaning & $\begin{array}{l}\text { Pr(meaning is ... I signal } \\
\text { is G-rep } 4)\end{array}$ \\
\hline$\left\{\mathrm{G}_{4}\right\}$ & $=4$ & $1 / 4$ \\
\hline$\left\{\mathrm{G}_{5}\right\}$ & $=5$ & $1 / 8$ \\
\hline$\left\{\mathrm{G}_{4}, \mathrm{G}_{5}\right\}$ & \pm 4 & $1 / 4$ \\
\hline$\left\{\mathrm{G}_{5}, \mathrm{G}_{6}\right\}$ & \pm 5 & $1 / 8$ \\
\hline$\left\{\mathrm{G}_{\mathrm{k}}: \mathrm{k} \geq 4\right\}$ & $\geq 4$ & $1 / 8$ \\
\hline
\end{tabular}




\begin{tabular}{|l|l|l|}
\hline$\left\{\mathrm{G}_{\mathrm{k}}: \mathrm{k} \geq 5\right\}$ & $\geq 5$ & $1 / 16$ \\
\hline$\left\{\mathrm{G}_{\mathrm{k}}: \mathrm{k} \geq 6\right\}$ & $\geq 6$ & $1 / 16$ \\
\hline
\end{tabular}

Finally, when we consider the strategic addressee $\mathrm{A}_{1}$, we will need to make assumptions about which meanings (according to them) the speaker $S_{1}$ is likely to express. These priors about the target meanings are given in (63), whose main property for our purposes is that 'at least' readings are more likely to be used than 'approximately' readings; lines with non-zero probabilities are boldfaced.

(63) Assumptions about priors: probability that $\mathbf{S}_{\mathbf{1}}$ wishes to express different meanings

\begin{tabular}{|l|l|}
\hline Target meaning & Prior probability \\
\hline $\mathbf{= 3}$ & $\mathbf{. 2}$ \\
\hline $\mathbf{= 4}$ & $\mathbf{. 2}$ \\
\hline $\mathbf{=} 5$ & 0 \\
\hline $\mathbf{+ 3}$ & $\mathbf{. 1}$ \\
\hline $\mathbf{4 4}$ & $\mathbf{. 1}$ \\
\hline $\mathbf{4 5}$ & 0 \\
\hline$\geq \mathbf{3}$ & $\mathbf{. 2}$ \\
\hline$\geq \mathbf{4}$ & $\mathbf{. 2}$ \\
\hline$\geq 5$ & 0 \\
\hline$\geq 6$ & 0 \\
\hline
\end{tabular}

\subsection{Three levels of communicative reasoning}

We start with the literal addressee $A_{0}$, whose behavior is given by (60)-(62): given a message, $A_{0}$ assigns one or several meanings to it, with probabilities that are entirely given in the latter case by perceptual properties of blurry pictures. ${ }^{29}$

Next, we consider the actions of the strategic speaker $S_{1}$, who selects the best message to convey the meaning they have in mind, taking into account the behavior of $\mathrm{A}_{0}$. We consider the boldfaced meanings in (63) and the 4 signals in (58). Since $S_{1}$ seeks to maximize the chance that the literal addressee $\mathrm{A}_{0}$ obtains the intended meaning $\mathrm{m}, \mathrm{S}_{1}$ 's chosen signal $\mathrm{s}^{*}$ should be an $\mathrm{s}$ that maximizes the probability $\operatorname{Pr}$ (meaning is $\mathrm{m}$ I signal is $\mathrm{s}$ ) in (60)-(62). This maximization condition is stated in (64) (in the case we consider, there will be a unique maximizer).

\section{(64) Behavior of the strategic speaker $S_{1}$}

Given a meaning $\mathrm{m}$, find a signal $\mathrm{s}^{*}$ such that

$\mathrm{s}^{*} \in \operatorname{Argmax}_{\mathrm{s}} \operatorname{Pr}($ meaning is $\mathrm{m} /$ signal is $\mathrm{s})\left(=\right.$ " $\mathrm{s}^{*}$ maximizes the value of $\operatorname{Pr}($ meaning is $\mathrm{m} /$ signal is $\mathrm{s}) ")$, or in other words:

$\operatorname{Pr}\left(\right.$ meaning is $m \mid$ signal is $\left.\mathrm{s}^{*}\right) \in \operatorname{Max}\{\operatorname{Pr}($ meaning is $\mathrm{m} /$ signal is $\mathrm{s})$ : $\mathrm{s}$ is a possible signal $\}$

It is clear that the meanings $=3$ and $=4$ are best conveyed by $G G G$ and $G G G G$ respectively. 'Approximately $\mathrm{n}$ ' and 'at least $\mathrm{n}$ ' readings are best conveyed by $G$-repn, for $\mathrm{n}=3$, 4.

\section{(65) Optimal choices for $\mathbf{S}_{\mathbf{1}}$ to convey different meanings}

\begin{tabular}{|l|l|}
\hline Target meaning & Optimal signal \\
\hline$=3$ & G G G \\
\hline$=4$ & G G G G \\
\hline \pm 3 & G-rep3 \\
\hline
\end{tabular}

${ }^{29}$ The reason we do things in this way is that we take $\mathrm{A}_{0}$ to be entirely naive and to just go by the iconic information she receives. Within a standard RSA model, one might expect the priors in (63) to already play a role at this point, in the following sense: the 'state of the world' that the addressee $\mathrm{A}_{0}$ seeks to recover is just the meaning that the speaker has in mind; $A_{0}$ has two sources of information about the state of the world (i.e. about the meaning the speaker seeks to convey): (i) the priors (= which meanings the speaker is likely to want to express); (ii) the information conveyed by (possibly blurry) repetitions. Here we solely consider (ii) at the level of $\mathrm{A}_{0}$. 


\begin{tabular}{|l|l|}
\hline \pm 4 & G-rep4 \\
\hline$\geq 3$ & G-rep3 \\
\hline$\geq 4$ & G-rep4 \\
\hline
\end{tabular}

At this point, punctuated repetitions give rise to unambiguous 'exactly' meanings, ${ }^{30}$ whereas unpunctuated repetitions are ambiguous: they can have 'approximately' or 'at least' readings. Importantly, the rest of the discussion will entirely derive from this result, and thus the details of the probabilities selected in (61)-(62) need not matter. In particular, the initial probability of an 'at least n' interpretation of G-repn could have been taken to be even lower, say $1 / 20$ instead of $1 / 8$, as long as $G$-repn remained the best way of conveying the 'at least n' meaning.

Finally, we consider the strategic addressee $A_{1}$, who takes into account the strategic speaker's behavior. On the basis of the priors pertaining to the meanings $S_{1}$ is likely to express, given in (63), $A_{1}$ will compute the probability of the meaning in view of the signal by using Bayes's rule, stated in (66):

(66) $\operatorname{Pr}$ (meaning is $\mathrm{m} /$ signal is $\mathrm{s}$ ) $=$

Pr(signal is $\mathrm{s} \mid$ meaning is $\mathrm{m}) * \operatorname{Pr}($ meaning is $\mathrm{m})$ $\Sigma_{\mathrm{m}^{\prime}} \operatorname{Pr}\left(\right.$ signal is $\mathrm{s}$ I meaning is $\left.\mathrm{m}^{\prime}\right) * \operatorname{Pr}\left(\right.$ meaning is $\left.\mathrm{m}^{\prime}\right)$

Using (63) and (65), we can compute the posterior probability of different interpretations by $\mathrm{A}_{1}$ of our four target expressions, as in (67):

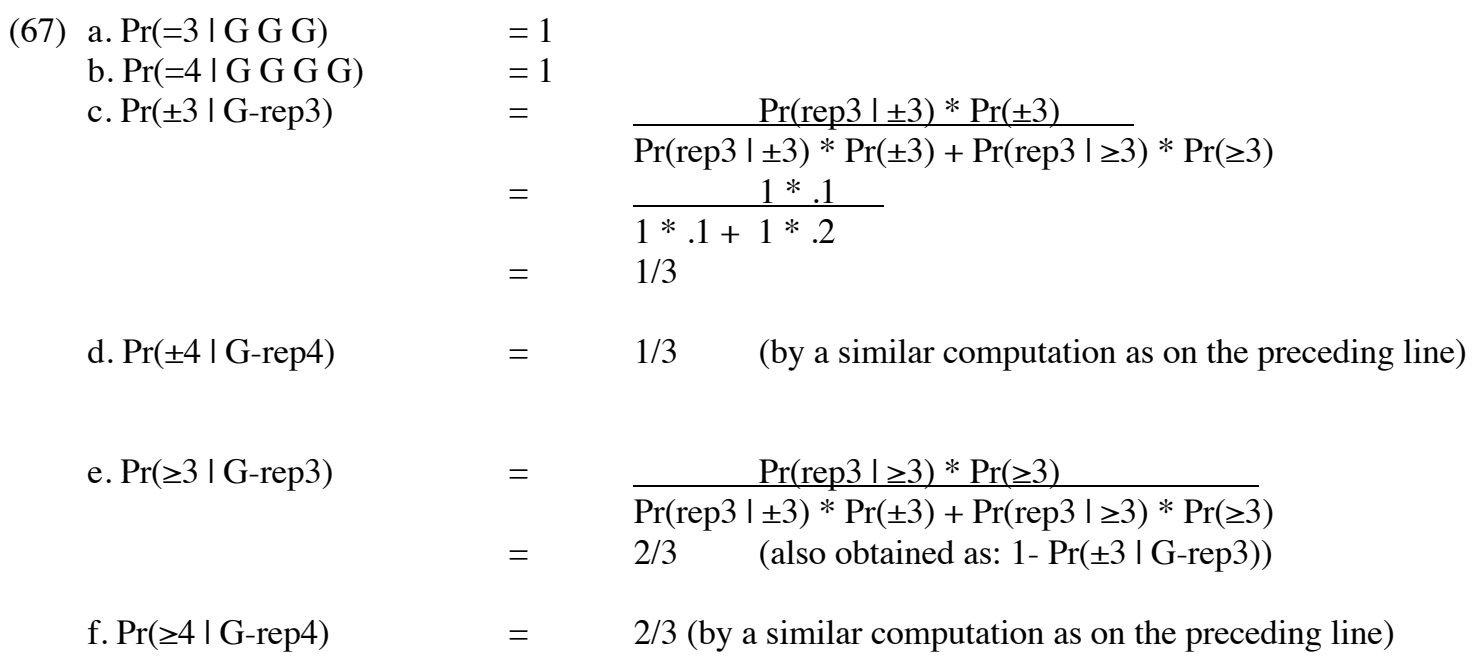

This gives rise to the interpretive strategy in (68).

(68) $\mathbf{A}_{1}$ 's interpretive strategy

\begin{tabular}{|l|l|l|}
\hline Signal & Meaning & Posterior probability $\operatorname{Pr}($ meaning I signal) \\
\hline G G G & $=3$ & 1 \\
\hline G G G G & $=4$ & 1 \\
\hline G-rep3 & \pm 3 & $1 / 3$ \\
\hline G-rep4 & \pm 4 & $1 / 3$ \\
\hline G-rep3 & $\geq 3$ & $2 / 3$ \\
\hline G-rep4 & $\geq 4$ & $2 / 3$ \\
\hline
\end{tabular}

We have thus solved our main problem, which was that the 'at least' reading was exceedingly unlikely on perceptual grounds. Thanks to strategic reasoning, it now becomes a rather likely meaning - and because we assumed (in the priors in (63)) that the speaker is more likely to be intending an 'at least' than an 'approximately' reading, this is the result we obtain in (68).

\footnotetext{
${ }^{30}$ We will refine the analysis in Section 9 to explain why separated slow repetitions give rise to an ambiguity (despite the fact that they are easy to count and should be classified as punctuated).
} 
It is worth noting that the relative weight of the 'approximately' and 'at least' interpretations of G-repn entirely stems from the priors in (63): the iconic implausibility of the 'at least' interpretation for $\mathrm{A}_{0}$ does not make itself felt any more. Concretely: since G-rep 3 is the optimal message for $S_{1}$ both in cases \pm 3 and $\geq 3$, we have that $\operatorname{Pr}(\operatorname{rep} 3 \mid \pm 3)=\operatorname{Pr}(\operatorname{rep} 3 \mid \geq 3)=1$, and as a result the lines in (67)c,e simplify to (69)a,b respectively:

(69) a. $\operatorname{Pr}( \pm 3$ | G-rep3 $)=\operatorname{Pr}( \pm 3) /(\operatorname{Pr}( \pm 3)+\operatorname{Pr}(\geq 3))$

b. $\operatorname{Pr}(\geq 3$ | G-rep3 $)=\operatorname{Pr}(\geq 3) /(\operatorname{Pr}( \pm 3)+\operatorname{Pr}(\geq 3))$

The priors in (63) specified that $\geq 3$ was twice as likely to be intended as \pm 3 , and this is the reason G-rep3 yields a posterior probability for an 'at least' interpretation of $2 / 3$ in (68). Had we picked priors on which \pm 3 and $\geq 3$ are equally likely, the posterior probability of the 'at least' interpretation for $A_{1}$ would have been $1 / 2$ (compared to $1 / 16$ for $A_{0}$ ).

We should add that a strategic speaker $S_{2}$ who determined the optimal signal in view of the interpretive behavior of $A_{1}$ in (68) would do the very same thing that $S_{1}$ did in (65), using punctuated repetitions in an unambiguous fashion (with an 'exactly' reading) and unpunctuated repetitions in an ambiguous fashion (with an 'approximately' or an 'at least' reading). Thus further levels of strategic reasoning will not be modify the results obtained at this point.

\subsection{Intermediate conclusion}

Several points should be highlighted. (i) First, despite the initial implausibility of an 'at least' reading of unpunctuated repetitions for the naive addressee $A_{0}$, it becomes highly available to the strategic addressee $A_{1}$. The reason is that an unpunctuated repetition is the best way for $S_{1}$ to convey the 'at least' reading. (ii) Second, an 'approximately' reading exists alongside the 'at least' reading, which is consistent with some of our data. (iii) Third, however, when an unpunctuated repetition is used, the 'at least' reading is more likely to be derived than the 'approximately' reading.

The first result (=(i)) does not hinge on the detailed priors we selected: the amplification of the 'at least' reading for unpunctuated repetitions is due to strategic reasoning. On the other hand, the last result (= (iii)) is 'baked into' the priors we selected in (63), and the fact that we took the speaker to wish to convey an 'at least' meaning more often than an 'approximately' reading. In that sense, our analysis of repetition-based plurals is not purely based on iconicity and cognition.

Finally, it should be mentioned that this highly simplified analysis has a flaw: it disregards the fact that producing 15 iterations when one aims for an 'exactly 15' or an 'at least 15 ' reading might be... taxing. For punctuated repetitions, this need not radically affect the analysis: excessive cost may lead one to avoid large numbers of punctuated repetitions, but when they are used, it is plausible that they usually get 'exactly' readings, and our model explains why this is. For unpunctuated repetitions, the problem is more severe: in cases (such as unembedded clauses) in which one can use an 'at least 3 ' reading (= 3 unpunctuated iterations) as well as an 'at least 15 ' reading (= 15 unpunctuated iterations), it is likely that one will go for the first option for reasons of cost. This problem, raised by a reviewer, is particularly sharp in production data from a different linguistic system, the home signs of some Deaf people Nicaragua. In the data of Coppola et al. 2013, different numbers of punctuated iterations are used for different 'exactly' readings (as we expect), but for what seem to be 'at least' readings, only 2-3 unpunctuated iterations are employed. We briefly discuss the problem as well as directions for a solution in Appendix II. 


\section{Modifying the marking rule?}

\section{$9.1 \quad$ A problem}

We return to the inferential data pertaining to embedding under $I F$-clauses in Section 4 . These pose a puzzle. On the one hand, unseparated repetitions give rise to a clear pattern: 4 unseparated fast iterations yield an at least 4 reading, as in (20)b, while 4 unseparated slow iterations give rise to an exactly 4 reading, as in (20)c. Setting aside separated fast iterations, which are always deviant (e.g. in (20)d), it is striking that separated slow iterations, as in (20)e, give rise to entirely unstable inferential judgments: they seem to be compatible with an at least or with an exactly reading. This is all the more surprising since, if anything, these iterations are easier to count than the unseparated slow repetitions which give rise to a clear exactly reading.

One could posit that these ambiguities are due to the optional presence of an existential quantifier over pluralities in the scope of the $I F$-clause. In a nutshell, starting from a reading akin to exactly 4 G's forming a circle as in (20)b, we could get an analysis such as (70), which just requires that it is possible to find a group of exactly $4 G^{\prime}$ s arranged in a circle. With this existential quantifier, the antecedent will remain true even if there are supernumerary G's in the drawing.

(70) If [ $\exists X: X$ is made of exactly $4 \mathrm{G}$ 's arranged in a circle] your drawing displays $\mathrm{X}$, I will give you $\$ 20$.

There are two reasons to doubt this account, however. The main worry is this: if the ambiguity of the separated slow iterations is due to the optional presence of an existential quantifier over pluralities within the $I F$-clause, why isn't the same possibility open with unseparated slow iterations as well? In our data, however, these do not give rise to instability in the judgments.

There is also a more minor worry. The predicate we used was LOOK-LIKE, which was designed to make the interpretation of the iterations as iconic as possible. Specifically, the analysis with an existential quantifier should really give rise to the truth conditions in (71).

(71) If [ $\exists X: X$ is made of exactly $4 \mathrm{G}$ 's arranged in a circle] your drawing looks like $X$, I will give you $\$ 20$.

But it is not entirely clear that these truth conditions are satisfied in a situation in which there are $5 \mathrm{G}$ 's arranged as a circle in the drawing. The reason is that in this case the drawing does not look like the iconic sign representation with the 4 separated slow iterations. At best, a (rather odd) subpart of the drawing looks like this iconic representation, which is a different thing.

\subsection{An iconic solution}

We propose that the problem we encountered is due to an ambiguity in the iconic conventions used by the signer. The question is what the absence of a marking in signing space corresponds to. Here we conjecture that there are two possibilities: if the hand does not appear in some area of signing space, this may be because no object projects onto that area, or because the representation is partial, i.e. says nothing at all about what may or may not project onto that particular area.

More specifically, we take the marking rule to have the following properties:

(i) if the signer's hand traces a circle or a $\$$ in an area, there should be an object with appropriate properties (corresponding to a circle, or to a $G$ ) that projects onto it;

(ii) if the signer's hand does not go down but does not trace anything, there should be no object that projects onto that area;

(iii) if the signer's hand goes down and thus does not appear at all in a certain part of signing space, there could be an ambiguity: either (1) there should be no object that projects onto that part of signing space, or (2) anything goes, in the sense that the representation is silent on that part of the manual picture. 
In the case of unseparated repetitions, the signer's hand does not go down between iterations of the circle or of the $\$$. While the representation may be underspecified with respect to parts of space in which the hand does not appear, for those parts in which the hand is up but does not trace anything, there should be no objects that project onto them.

In the case of separated repetitions, the hand goes down between the iterations. This gives rise to the following ambiguity pertaining to the space found between the iterations: according to (iii)(1), there should be no object with relevant properties that projects onto that space, hence an 'exactly' readings (= the objects arranged in a circle are exactly those that are depicted in signing space); according to (iii)(2), the representation is silent about the space that is found between the iterations, hence an 'at least' reading ( $=$ the objects arranged in a circle include at least those that are depicted in signing space).

If we write as a continuous line the parts of signing space in which the hand is up, we have the result depicted in (72)a: apart from the 4 circles traced in the representation, no object should project onto the continuous line, and this blocks a situation in which 5 rather than 4 circles are arranged in a circular shape. When the hand goes down between iterations, as in (72), a further reading arises, one on which the representation is silent concerning points of signing space in which the hand goes down (if we apply the rule in (iii)(2) above).

(72) Possible denotations with a liberal marking rule on which the representation is silent when the hand goes down

a. The hand doesn't go down between the repetitions of a circle

Array in signing space

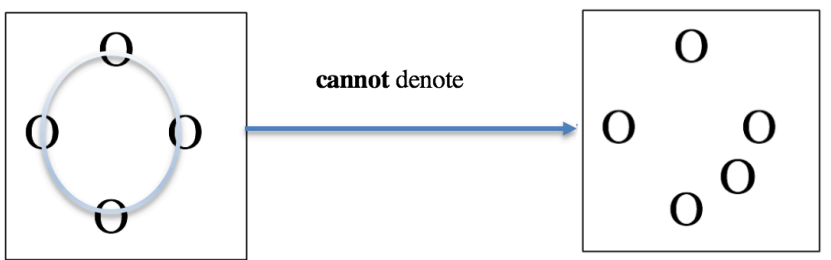

b. The hand goes down between the repetitions of a circle
Drawing described

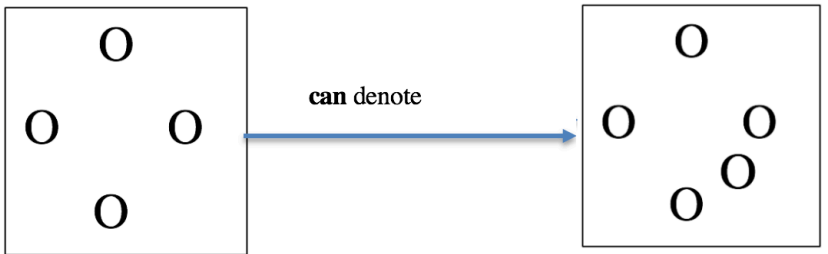

Thus we propose to account for the ambiguity of separated but not unseparated repetitions by way of the ambiguity of the marking rule as specified in (73).

(73) Marking rule for iconic sign representations

A manual projective feature map is accurate at a scene $s$ if and only if, for each atomic part $p$ of the array,

a. Strict version

$\mathrm{p}$ is manually marked with feature $\mathrm{F}$ if and only if some object with feature $\mathrm{F}$ in s projects onto $\mathrm{p}$

b. Liberal version

if the hand goes through an area surrounding $p, p$ is manually marked with feature $F$ if and only if some object with feature $\mathrm{F}$ in s projects onto $\mathrm{p}$. 
On the strict version, if the hand fails to mark the array in signing space with a feature, i.e. it fails to trace anything, no object should project onto it. This holds whether the hand is up or down in the relevant part of space. On this strict marking rule, then, the separated repetitions on the lefthand side of (72)b cannot denote the situation (itself a drawing) that appears on the right-hand side. On the liberal marking rule, on the other hand, the 4 separated iterations can denote the picture with 5 circles because the picture drawn in signing space is silent on those parts of the array in which the hand goes down. On both the strict and the liberal rule, the 4 unseparated iterations in (72)a fail to denote the drawing with the 5 circles on the right.

Importantly, the liberal version doesn't require that the hand go through an atomic part $\mathrm{p}$ for the 'if and only if' condition to be in force, but only through an area surrounding $p-$ an expression which is left intentionally vague. The reason we use through an area surrounding $p$ rather than through $p$ is that otherwise our rule would be too weak. Consider the bunny at the top of the drawing on the right-hand side of (74). It includes an $\mathrm{O}$ as a proper part. Thus if the marking rule made it possible to select arbitrarily what is represented and what is omitted, the representation on the left should be deemed accurate (it would just happen to be silent about the eyes, nose and ears of the bunny). Intuitively, this is the wrong result.

(74) A possible denotation relation on an excessively liberal marking rule

Array in signing space

Drawing described

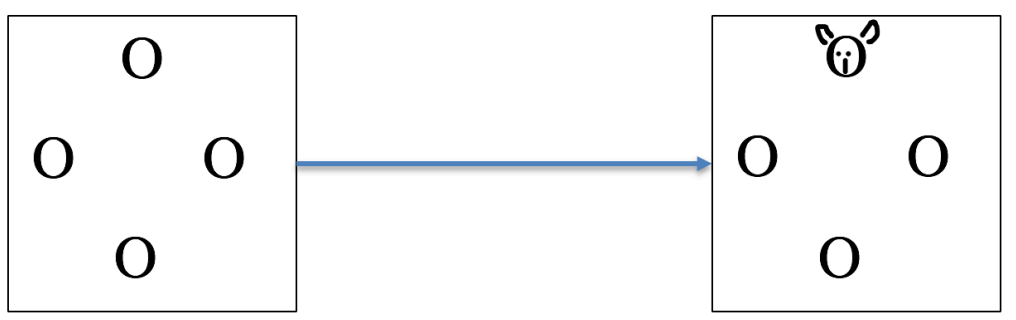

The requirement that the 'if and only if' condition should hold as soon as the hand goes through an area surrounding $p$ is intended to block this case: since the hand depicts the top-most $O$, it should also depict any edges surrounding it, hence also the eyes, nose and ears of the bunny. Of course this condition would have to be made more precise in future work.

In sum, we have a possible solution to our puzzle. The problem was that separated repetitions are more ambiguous (between an 'exactly' and an 'at least' reading) than unseparated repetitions, despite the fact that the former are easier to count than the latter. We proposed a solution that crucially involves the details of a pictorial semantics. The main point is that when the hand goes down in a part of the array, this could be for two separate reasons depending on the marking rule: because no object projects onto that part of the array, or because the representation is silent on that part of the array. When the hand is up but fails to trace anything, no ambiguity arises, as both marking rules require that no object project onto the relevant part of the array.

One issue we leave for future research is the development of a proper account of examples such as those in (75) (discussed in Schlenker and Lamberton 2019), where the numerals 4 or 10 co-occur with three punctuated iterations of TROPHY.

(75) MUSEUM HAVE

a. ${ }^{7} 4$ [TROPHY TROPHY TROPHY $]_{\text {horizontal_arc. }}$

'The museum has 4 trophies (spread out).'

b. ${ }^{7} 10$ [TROPHY TROPHY TROPHY $]_{\text {horizontal_arc. }}$

'The museum has 10 trophies (spread out).'

(Schlenker and Lamberton 2019, ASL 34, 2216, f, h; 3 judgments. Anonymized video of the entire paradigm: https://youtu.be/029qrrytaPQ. Note that a. and b. are the 6th and 8th examples; see Schlenker and Lamberton 2019 for a transcription of the full paradigm.) 
Importantly, in this case the iterations are realized as unseparated and slow, and yet the number of trophies depicted is probably not interpreted literally, or else it would clash with the numerals. ${ }^{31}$ The pictorial component seems to contribute the information that the trophies are spread out. One possibility that the repetitions are in this case a pictorial representation of a subpart of the denoted group (maybe because part of the objects are hidden from view); but why this is and how this phenomenon should be constrained remains to be seen. ${ }^{32}$

\section{Possible extensions}

Our analysis of repetition-based plurals in sign language relies on three modules: pictorial semantics, vagueness, and pragmatic exploitation (which interacts with a linguistic bias for some salient readings). All three modules could exist in a variety of communicative situations, and thus repetition-based plurals should be found in a variety of iconic forms. After discussing experimental data that might dovetail with our results, we consider potential extensions to sign language pluractionals, to repetition-based plurals and pluractionals in gestures, to vocal repetitions, and to pictures.

\subsection{Connection with experimental data on classifier predicates ${ }^{33}$}

As noted by a reviewer, our conclusions on 'exactly' readings of punctuated repetitions (especially unseparated slow repetitions) dovetail with experimental results from Davidson and Mayberry 2015. In a paper devoted to scalar implicatures in ASL, they used as a control two or three iterations of an animal classifier (transcribed as $C L: 5($ claw)) in different parts of signing space, to represent either two bears, as in (76), or three bears (with one additional iteration of the classifiers).

(76) BEAR CL:5(claw) $)_{Y}$, CL:5(claw)Z.

'There is a (toy) bear here (at location Y) and here (at Z).'

${ }^{31}$ This depends on one's specific view on numeral semantics. The problem we refer to will arise with the rather natural semantics in (i), in which the effect of the numeral 4 is to introduce a maximal group of objects that satisfy the noun and the predicate (this is akin to the 'reference set' discussed in connection with anaphora in Nouwen 2003, Chapter 3). If $N$-iter is made of 3 slow unseparated iterations of TROPHY, it could not be a picture (part) true of a group of exactly 4 trophies, unless some trophies are somehow hidden.

(i) $\left[\left[[4 \mathrm{~N} \text {-iter }]_{\mathrm{x}} \mathrm{P}\right]\right]^{\mathrm{c}, \mathrm{s}}=$ true iff for some group $\mathrm{d}$,

(a) $d$ is the maximal group d' such that $\left[[N]^{c, s\left[X \rightarrow d^{\prime}\right]}\left(d^{\prime}\right)=\right.$ true and $\left[[P]^{c, s\left[X \rightarrow d^{\prime}\right]}=\right.$ true,

(b) d contains exactly four objects, and

(c) $N$-iter is tolerantly true of $\mathrm{d}$.

${ }^{32}$ This analytical direction need not conflict with the marking rule in (73)b if the 3 iterations of TROPHY depict a (possibly typical) subpart of the denoted group. In (75)a,b, the hand does not go down between the iterations, and this is presumably responsible for the inference that the trophies are spread out (by (73)b, the areas through which the hand goes without tracing TROPHY are ones in which there is nothing, hence the 'spread out' inference). Things are different in (72)b, where the hand goes down between the iterations, with the result that the marking rule in (73)b is silent about what there is between the depicted objects.

${ }^{33}$ Many thanks to a reviewer for Linguistics \& Philosophy for highlighting the relevance of the references mentioned in this section. 


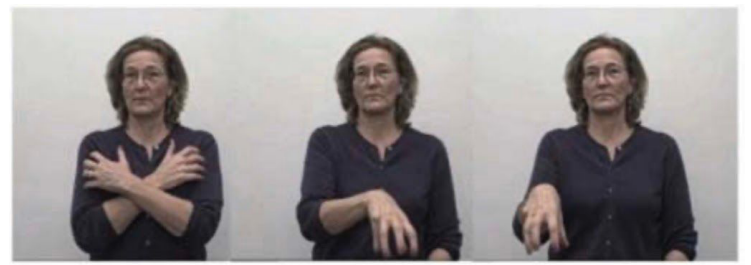

(Davidson and Mayberry 2015, Figure 2b)

ASL subjects included two populations: early Deaf learners, who strongly computed the 'not all' implicatures triggered by SOME (which forms a scale with ASL $A L L$ ), and late (non-native) Deaf learners, who computed this implicature less strongly, thus endorsing some under-informative sentences. Strikingly, both populations were at ceiling with respect to the 'exactly two' reading of the classifier in (76): if there were three objects on the table and a sentence described the situation with just two iterations of the classifier, it was rejected $100 \%$ of the time by early learners and $98 \%$ of the time by late learners. One possible interpretation is that this 'exactly' reading just isn't due to a scalar implicature but is the literal meaning of the construction. On this view, this is an 'exactly' reading because the meaning is based on a pictorial semantics, and objects that fail to be represented are likely absent.

Relevant as well are experimental results from Davidson 2014, who compared underinformative sentences in English and in ASL. In both languages, subjects were presented with a situation with three objects, only two of which were mentioned. Thus in the presence of a candle, a globe and a wallet, they had to assess an English sentence such as There is a candle and a globe on the table. ASL native signers were asked to assess an ASL near-analogue, but one that crucially involved classifiers for the nouns. ${ }^{34}$ In this case, there was "quantitatively a higher percentage" of rejections of the uninformative sentences in ASL (88\% rejection) than in English (54\% rejection; the difference between English and ASL was "marginally significant"). One possibility entertained by Davidson is that there is in this case a difference between the two languages in terms of the derivation of ad hoc implicatures. An alternative that could be investigated is that in ASL the 'exactly' reading is due to pictorial semantics, a different mechanism..$^{35}$

More generally, predicate classifiers are usually treated as lexical forms whose position or movement in signing space is interpreted highly iconically (e.g. Emmorey and Herzig 2003, Zucchi 2011, Davidson 2015). It would be natural in the future to extend to predicate classifiers (not just repeated ones, as in Davidson and Mayberry's work) the pictorial semantics developed in this piece. ${ }^{36}$

${ }^{34}$ An ASL sentence cited in part in Davidson 2014 (Figure 3a) appears in (i):

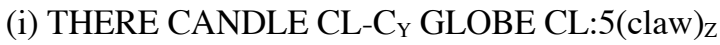

'There is a candle and a globe.'

${ }^{35}$ Davidson 2014 sketches an account based on the interaction between Questions under Discussion and classifiers (which bias the question towards the configuration of the objects). This is congenial to but distinct from the pictorial analysis envisaged here. As in our discussion of (75), however, one issue to keep in mind is that a picture need not depict the entirety of the situation if some objects are hidden from view; this might allow some ASL native signers to accept the under-informative statement.

${ }^{36}$ Tangentially, a reviewer asks about further uses of repetitions in ASL. Lamberton can think of these: (i) First, a case that is almost certainly unrelated to the present investigation: a single repetition (i.e. a total of 2 iterations) is a standard and well-known nominalization strategy in ASL, and serves for instance to distinguish CHAIR (nominal, 2 iterations) from SIT (verbal, 1 iteration). (ii) As in English, repetition can serve to intensify, as in I never never never did that. (iii) Far closer to our topic, there are temporal uses of repetitions: to say that someone won many trophies in their career, one might sign TROPHY repeatedly from one's shoulder down to a neutral space - hence: many trophies were won over time. We leave these diverse uses for future research. 


\subsection{Sign language pluractionals}

In sign languages such as ASL and LSF, repetitions can be used with a pluractional meaning (referring to a plurality of events rather than entities). Kuhn 2015 particularly focuses on the interaction between logical and iconic conditions in this domain. He thus discusses examples such as (77), where GIVE is repeated at an accelerating pace to refer to an action that accelerates; Kuhn and Aristodemo 2017 make similar remarks about LSF.

(77) IF JOHN PAPERS GIVE-alt-speeding-up, SECRETARY WILL HAPPY.

'If John gives papers at an accelerating rate, the secretary will be happy.' (ASL, Kuhn 2015)

Focusing on ASL, Fischer 1973 notes the existence of two varieties of repetition-based pluractionals, which she calls 'slow' and 'fast' reduplication. These might correspond to what is termed punctuated and unpunctuated repetitions, but unfortunately we know of no detailed semantic investigation of the interpretive differences between them, and thus this is a topic we must leave for future research (but see relevant discussions of verbal reduplication in Klima and Bellugi 1979, Cormier 2002, Liddell 2003, among others).

\subsection{Repetition-based plurals and pluractionals in gestures}

Schlenker and Lamberton 2019 and Schlenker, to appear a argue that the distinction between punctuated and unpunctuated repetitions can be replicated with gestures that have a plural or a pluractional reading (see Feldstein 2015 for an earlier attempt to study plurals in gestures). Based on a detailed survey with three consultants, Schlenker, to appear a discusses in particular the contrast in (78), pertaining to plurals, and that in (79), pertaining to pluractionals.

Notation: CROSS represents the gesture

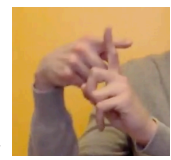
in a punctuated fashion for CROSS CROSS CROSS, in an unpunctuated fashion for CROSSrep3.

SLAP is a slapping gesture produced in neutral position (in front of the speaker). SLAP, SLAP, SLAP encodes three repetitions with clear pauses, SLAP-repm encodes n repetitions without clear pauses; accelerating and decelerating indicate that the repetitions are produced at an accelerating and decelerating speed respectively. (Judgments are averaged over 3 consultants on a 7-point scale, with $7=$ best.)

(78) Context: The addressee is taking part in a treasure hunt in churches. The speaker provides an indication about the location of the treasure.

a. ${ }^{6.7}$ If you enter a room and you see [CROSS CROSS CROSSI horizontal, you have reached the prize.

$\Rightarrow>$ when he reaches the prize, the speaker will see:

exactly three crosses (precise)

arranged horizontally

b. ${ }^{5}$ If you enter a room and you see CROSS-rep3 $3_{\text {horizontal }}$, you have reached the prize.

$\Rightarrow>$ when he reaches the prize, the speaker will see:

? three crosses or more (imprecise)

arranged horizontally

(Video 4001, examples a. and c.

(79) My opponent, I am going to

a. ${ }^{5.7}$ SLAP, SLAP, SLAP.

$\Rightarrow$ the speaker will slap his opponent: 
exactly three times

slowly [ $3 / 3$ consultants $]$ / in a very deliberate fashion [ $1 / 3$ consultants $]$

b. ${ }^{5.3}$ SLAP-rep3.

$\Rightarrow$ the speaker will slap his opponent:

two/three times [2/3 consultants] / exactly three times [1/3 consultants] / three times or more [1/3

consultants]

fast

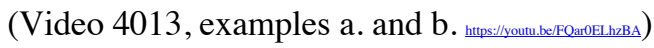

If confirmed, these and other data (pertaining to the arrangement of the iterations in iconic ways) display a striking similarity to the sign language data in Schlenker and Lamberton 2019. This similarity is expected from the present perspective, but requires a more detailed empirical investigation.

\subsection{Repetitions in the vocal modality}

\subsubsection{Onomatopoeias $^{37}$}

The distinction between punctuated and unpunctuated repetitions can arguably be replicated in the vocal medium. In the context of a shooter video game, one may use an onomatopoeia akin to prh to evoke a gunshot. But our impression is that different quantitative implications are obtained depending not just on the number of repetitions, but also on their realization, as illustrated in (80): if 4 iterations are separated by pauses and easy to count, one can get an 'exactly four' reading. If the 4 iterations are produced without pauses and are harder to count, one might get instead an 'approximately four' or an 'at least four' reading.

(80) Context: the addressee is playing a shooter video game.

a. In the next 10 seconds, you should [prh - prh - prh - prh] $]_{\text {slow, pauses }}$

b. In the next 10 seconds, you should prh-rep4 $4_{\text {fast, no pauses }}$

c. If in the next 10 seconds you [prh - prh - prh - prh] slow, pauses, I'll give you \$20.

d. If in the next 10 seconds you prh-rep4 $4_{\text {fast, no pauses }}$, I'll give you $\$ 20$

We describe in Appendix III a mini-survey (with 6 linguists) which was designed to test these impressions, with inferential questions that were very close to those we used in ASL. The findings pertaining to punctuated repetitions indeed argue for the existence of 'exactly' readings, including in the scope of if. The results on unpunctuated repetitions are harder to analyze, in part because several consultants interpreted them as involving a machine gun in which pulling the trigger once gives rise to a burst of shots. When this is taken into account, it seems that 'at least 4 ' readings were sometimes obtained, although additional readings appeared as well (possibly including an 'at least 1 ' reading). Further details can be found in Appendix III. ${ }^{38}$

\subsubsection{Repetition-based plurals and pluractionals in speech}

As mentioned in Pfau and Steinbach 2006 and in Kuhn and Aristodemo 2017, reduplication is a common means of plural and pluractional formation in spoken language, as illustrated in (81).

\footnotetext{
37 Thanks to Jeremy Kuhn (p.c.) for helpful remarks.

${ }^{38}$ Several directions could be explored in future research. First, as suggested by J. Kuhn (p.c.), it would be useful to create stimuli by splicing one and the same realization of an onomatopoeia in several linguistic contexts so as to ensure that the repeated sounds in (80)a,b (or (80)c,d) are genuinely identical. Second, it would be useful to investigate nominal (rather than pluractional) uses of repeated onomatopoeias so as to have a possibly closer point of comparison for ASL plurals. In a different context, Guerrini and Migotti 2019 used repeated onomatopoeias to evoke several champagne bottles (the sound was evocative of a cork popping). We are not sure how to realize these sounds in an unpunctuated fashion, but this direction would be worth exploring.
} 
(81) a. Plural formation by reduplication

Warlpiri: $k u r d u$ 'child' $=>$ kurdu-kurdu 'children' (Pfau and Steinbach 2006, citing Nash 1986)

b. Pluractional formation by reduplication

Hausa: kiraa 'call' => kirkiraa 'keep on calling'/‘call many people' (Kuhn and Aristodemo 2017, citing Newman 2012)

It would be interesting to explore in the future what relation, if any, these bear to the repetitionbased plurals discussed in this piece.

\subsection{Comics and cartoons}

We conjecture that hard-to-count iterations might be used in comics to evoke pluralities of indefinite numerosity. A possible example is reproduced in (82), where the number of coins appearing at the surface of the 'sea of money' is indeterminate, but large, and greater than the number of individual coins that can be distinguished (see Cohn 2013, chapter 2, for other uses of repetition/reduplication in comics).

(82) Logo for Disney's Ducktales ${ }^{39}$

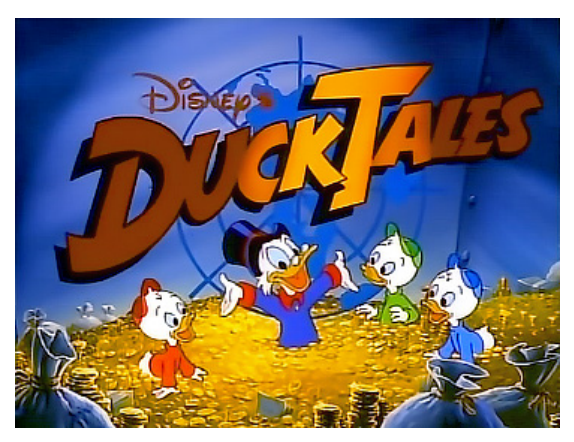

\section{Conclusion}

\subsection{Main results}

We started with a puzzle: the distinction between punctuated and unpunctuated repetitions seems to have arisen repeatedly across signs and gestures, in some cases without obvious input. Within ASL, the distinction arises in purely iconic representations just as it does in words, including in cases in which the repetition involves a gradual modification of the repeated shape (unlike in standard grammatical cases). A related distinction can arguably be found in other media, such as vocal onomatopoeias. While these observations could lead to the conclusion that, in this domain, Universal Grammar specifies a specific mapping between form and meaning, we developed an alternative account based on the interaction between different modules.

In our final analysis, an iconic semantics was combined with a theory of pictorial vagueness to yield multiple possible readings (depending on the set of precisifications considered), including an 'exactly' reading for punctuated repetitions, and an extremely unlikely 'at least' reading akin to plurals for unpunctuated repetitions. By pragmatic reasoning, the latter possibility was exploited for communicative purposes, making it dominant in some cases (while still allowing for an 'approximately' reading). More speculatively, we argued that an unexpected ambiguity found with slow separated iterations (but not with unseparated iterations) can be traced to the details of the 'marking rule' used in pictorial semantics.

Two broader conclusions can be drawn. First, a full understanding of linguistic meaning requires a detailed theory of iconic representations (in fact, of blurry iconic representations), including in an area thought to be as grammatical as plurals. Second, there might be a counterpart

\footnotetext{
${ }^{39}$ Retrieved on November 23, 2019 from https://en.wikipedia.org/wiki/DuckTales\#/media/File:DuckTales (Main title).jpg.
} 
of plurals in non-linguistic systems because the combination of means used in unpunctuated repetitions (iconicity, vagueness, pragmatic exploitation) are available more broadly.

\subsection{Open questions}

This article leaves several questions open. On an empirical level, our data ought to be tested with further ASL consultants, and extended to other sign languages. It would also be interesting to see if similar distinctions can be found in repetition-based pluractionals - something we sketched for gestural pluractionals but not for sign pluractionals. ${ }^{40}$ Various extensions beyond signs and gestures should be considered as well, as we sketched for vocal onomatopoeias and comics (music could be considered too). Two components of our analysis particularly require further investigation: the existence of 'approximately' readings in downward-monotonic environments; and the subtle semantic distinction between unseparated and separated slow repetitions.

On a theoretical level, several important questions require further research. First, for reasons of simplicity we adopted a bivalent tolerant interpretation of blurry pictures, but this eliminates vagueness per se (which would require that unclear cases be deemed 'neither true nor false' or 'both true and false'). As a result, both 'approximately' and 'at least' readings came with clear numerical conditions, which seems inaccurate (there should be clear and unclear cases, as in genuine theories of vagueness). More sophisticated accounts ought to be explored. The pragmatic component of our analysis was also reduced to its bare bones: besides assuming perfect rationality, we made rather strong assumptions about the meanings considered (only 'exactly', 'approximately' and 'at least' readings); in effect, this implemented a linguistic bias in pictorial semantics/pragmatics, since in principle many further potential readings could have been considered. It would be interesting to investigate what happens when these strong assumptions are relaxed. Finally, one should explore how more standard RSA models could account for our data.

\footnotetext{
${ }^{40} \mathrm{We}$ also said nothing about the potential role of the speed of repetition per se. In Schlenker and Lamberton 2019 , it was mentioned that, all other things being equal, greater speed might be associated with larger quantities. In the present theory, speed only plays an indirect role, by making the iterations harder to count. The theory might have to be refined to account for a more direct relation between speed and quantities.
} 


\section{Appendix I. Blurry pictures: truth via entailments vs. bivalent tolerant truth}

We reiterate in (83) our definition of bivalent tolerant truth for blurry pictures, and in (84) the definition of truth via entailments for blurry pictures. For readability we boldface propositions, and thus if $P$ is a picture (evaluated relative to a viewpoint $\mathrm{v}$ and a system of projection $\mathrm{S}$ ), we will write as $\boldsymbol{P}$ the set of worlds that make $P$ true.

(83) Bivalent tolerant truth for a blurry picture

Relative to a world w, a viewpoint $\mathrm{v}$ and a system of projection $\mathrm{S}$, a picture $P$ associated with precisifications $\pi(\mathrm{P})=\left\{\mathrm{P}_{1}, \mathrm{P}_{2}, \ldots\right\}$ is tolerantly true iff for at least one $\mathrm{P}_{\mathrm{i}}$ in $\pi(\mathrm{P}), \operatorname{proj}_{\mathrm{s}}(\mathrm{w}, \mathrm{v})=\mathrm{P}_{\mathrm{i}}$; tolerantly false otherwise.

(84) Truth via entailments - case of a blurry picture

Relative to a world $\mathrm{w}$, a viewpoint $\mathrm{v}$ and a system of projection $\mathrm{S}$, a picture $P$ associated with precisifications $\pi(\mathrm{P})=\left\{\mathrm{P}_{1}, \mathrm{P}_{2}, \ldots\right\}$ is true* iff for each proposition $\mathbf{E}$ such that $\mathrm{P}$ entails $\mathbf{E}, \mathbf{E}$ is true in w; false* otherwise.

In this case, bivalent tolerant truth yields the same result truth via entailments, as stated in (85).

(85) Let $P$ be a picture evaluated relative to a world $\mathrm{w}$, a viewpoint $\mathrm{v}$ and a system of projection $\mathrm{S}$, and let $\pi(\mathrm{P})=\left\{\mathrm{P}_{1}, \mathrm{P}_{2}, \ldots\right\}$ be the set of its precisifications.

$P$ is true* in $\mathrm{w}$ if and only if $P$ is bivalently tolerantly true in $\mathrm{w}$.

Proof

Relative to a viewpoint $\mathrm{v}$ and a system of projection $\mathrm{S}$ :

$\mathrm{P}$ is true* in $\mathrm{w}$ iff [1] for each proposition $\mathbf{E}$ such that $\mathrm{P}$ entails $\mathbf{E}, \mathrm{w} \in \mathbf{E}$

iff [2] for each proposition $\mathbf{E}$ such that for each precisification $\mathrm{P}^{\prime}$ of $\pi(\mathrm{P}), \mathrm{P}^{\prime}$

entails $\mathbf{E}$, w $\in \mathbf{E}$

iff [3] for each proposition $\mathbf{E}$ such that for each precisification $\mathrm{P}^{\prime}$ of $\pi(\mathrm{P}), \mathbf{P}^{\prime}$

$\subseteq \mathbf{E}, \mathrm{w} \in \mathbf{E}$
iff $\quad[4] \mathrm{w} \in \cup_{\mathrm{P}^{\prime} \in \pi(\mathrm{P})} \mathbf{P}^{\prime 41}$
iff $\quad[5]$ for some $\mathrm{P}^{\prime} \in \pi(\mathrm{P}), \mathrm{w} \in \mathbf{P}^{\prime}$
iff [6] $\mathrm{P}$ is tolerantly true in $\mathrm{W}$.

\footnotetext{
${ }^{41}$ In greater detail: Assume w satisfies [3]. Setting $\mathbf{E}=\cup_{\mathrm{P}^{\prime} \in \pi(\mathrm{P})} \mathbf{P}^{\prime}$, we have that for each precisification $\mathrm{P}^{\prime}$ of $\pi(\mathrm{P}), \mathrm{P}^{\prime}$ entails $\mathbf{E}$, hence $\mathrm{w} \in \mathbf{E}$, i.e. [4]. Now assume [4]. Thus for some $\mathrm{P}^{*} \in \pi(\mathrm{P}), \mathrm{w} \in \mathbf{P}^{*}$. It follows that for each proposition $\mathbf{E}$ such that for each precisification $\mathrm{P}^{\prime}$ of $\pi(\mathrm{P}), \mathbf{P}^{\prime} \subseteq \mathbf{E}, \mathbf{P}^{*} \subseteq \mathbf{E}$ and therefore $\mathrm{w}$ $\in \mathbf{E}$.
} 


\section{Appendix II. On Nicaraguan homesigners ${ }^{42}$}

Both the iconic and the pragmatic component of our analysis could be expected to apply to other systems, in particular other sign languages, and also homesigns. As a reviewer notes, the production data of Nicaraguan homesigners reported in Coppola et al. 2013 might seem to raise a worry for our analysis. In a production experiment, homesigners "were videotaped describing ten 10- to 20-s vignettes with varying numbers of objects and events". The authors coded three types of number marking: "Finger Extensions (FEs) were handshape configurations in which each extended finger indicated a member of the target set"; "Punctuated Movements (PMs) were a series of discrete movements, each referring to an entity or action in the vignette"; "Unpunctuated Movements (UMs) were movements produced in rapid succession with no clear break between them". The latter two categories seem to correspond rather closely to the punctuated and unpunctuated repetitions we described for ASL. In view of the model of Section 8, we would expect that, when they are used, punctuated repetitions should only have 'exactly' reading. This is roughly consistent with the data in (86): when punctuated movements were used, there were roughly as many iterations as there were objects to describe. What might be concerning, on the other hand, is that the number of unpunctuated iterations does not seem to depend on the number of objects to be described.

(86) Use of finger extensions (FE), punctuated movements (PM) and unpunctuated movements (UM) in a production experiment on Nicaraguan homesigners (Coppola et al. 2013)

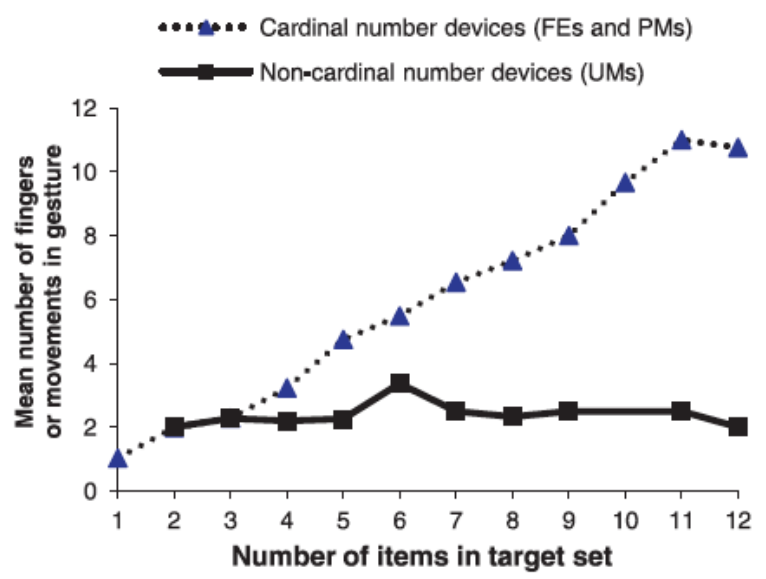

There are two issues to be addressed. One is the apparent absence of 'approximately' readings of unpunctuated repetitions. Since this is not the dominant reading we derive in (68) (in essence, because we postulated that such a reading would be used less often than the 'at least' reading), we might posit that it is either unavailable or not deemed useful in this production situation and/or in this language. With 2-3 unpunctuated iterations, the 'at least' readings we derive in (68) are correctly predicted to be true in the situations in which they are used (for present purposes, we treat two unpunctuated iterations along the same model as the three unpunctuated iterations, with an 'at least 2 ' reading). But this leaves open another question: why do subjects not produce 'at least 3 ' readings (with 3 unpunctuated iterations) when there are 3 objects or more, 'at least 4' readings (with 4 unpunctuated iterations) when there are 4 objects or more, etc?

There are several possible directions to explore; but an important general lesson is that we would need to take into account cost differences between different unpunctuated repetitions: when $\mathrm{n}<\mathrm{m}$, repn (meaning 'at least $\mathrm{n}$ ') is both less informative and less costly than repm (meaning 'at least $\mathrm{m}$ '), which involves more iterations.

\footnotetext{
${ }^{42}$ Many thanks to a reviewer for Linguistics \& Philosophy for raising questions addressed in this appendix.
} 
(i) One possibility to explain the homesign data is that the latter just don't allow for more than 23 unpunctuated iterations. But from the perspective of our iconic analysis, this is entirely stipulative.

(ii) Another possibility is that the preference for minimal numbers of unpunctuated repetitions is an effect of the task. We could reason as follows: the subjects are asked to describe a scene. If they think that precision matters, they will go for 'exactly' readings and thus for punctuated repetitions. If they think that precision doesn't matter, they will go for the least costly (i.e. shortest) unpunctuated repetition compatible with the scene, hence using in effect 'at least 2-3' whenever this is true, including for large numbers of objects to describe. On this view, the same results would be expected to arise with ASL-signing subjects faced with the same task.

(iii) Yet another possibility is that there is a difference between homesigns and ASL with respect to the cost of the iterations, with more iterations being somehow more costly in homesigns than in ASL. Why this should be is unclear, however.

This question ought to be further investigated in more sophisticated models than the one developed in this piece. 


\section{Appendix III. Mini-survey on repeated onomatopoeias}

We repeat in (87) the target paradigm involving repeated onomatopoeias, from (80).

(87) Context: the addressee is playing a shooter video game.

a. In the next 10 seconds, you should [prh - prh - prh - prh] slow, pauses.

b. In the next 10 seconds, you should prh-rep4 $4_{\text {fast, no pauses. }}$.

c. If in the next 10 seconds you [prh - prh - prh - prh] slow, pauses, I'll give you $\$ 20$.

d. If in the next 10 seconds you prh-rep4 $4_{\text {fast, no pauses, }}$, I'll give you $\$ 20$.

We tested this paradigm by way of audio files and an online survey with 6 consultants (all native speakers of American English, all linguists, including one working on gestures and one working on sign language). ${ }^{43}$ Acceptability and (open or quantitative) inferential questions were modeled after those we used in ASL, with quantitative questions displayed in (88). Average acceptability scores (with $7=$ best) and inferential scores (with $7=$ strongest) are reported in (89). They should be interpreted with caution because there was a lot of variation among consultants (see the Supplementary Materials).

(88) Questions

a. Acceptability: How acceptable is the sentence you heard on a 7-point scale (with $1=$ worst, $7=$ best)?

\section{b. Quantitative inferential questions for $(80) a, b$}

How strongly do you draw the following inferences? (with $1=$ no inference; $7=$ strongest inference)

In the next 10 seconds, the addressee should shoot...

...exactly 4 times.

... approximately 4 times.

....at least 4 times.

....at least 5 times.

\section{c. Quantitative inferential questions for $(80)$ c,d}

Assuming that the shots are spaced appropriately (if relevant), how strongly do you infer that the speaker is committed to giving the addressee $\$ 20$ in case the addressee shoots...

...exactly 4 times.

....approximately 4 times.

....at least 4 times.

....at least 5 times.

(89) Inferential results (averages, mini-survey of 6 linguists, see the Supplementary Materials)

Dominant inferences (i.e. those with the highes endorsement for a given sentence) are boldfaced.

\begin{tabular}{|l|l|l|l|l|l|}
\hline \multirow{2}{*}{ Sentences } & \multirow{2}{*}{ Acceptability } & \multicolumn{5}{|l|}{ Number of objects that the repetitions stand for } \\
\cline { 3 - 6 } & & $=4$ & \pm 4 & $\geq 4$ & $\geq 5$ \\
\hline a. $[$ prh - prh - prh - prh] slow, pauses & 5.5 & $\mathbf{5 . 7}$ & 3.7 & 4.8 & 1 \\
\hline b. prh-rep4 fast, no pauses & 6.5 & 2 & 3.3 & $\mathbf{3 . 8}$ & 2 \\
\hline c. [prh - prh - prh - prh]slow, pauses & 5.2 & $\mathbf{5 . 7}$ & 2.3 & 4.5 & 3.2 \\
\hline d. prh-rep4 $4_{\text {fast, no pauses }}$ & 6 & $\mathbf{4 . 5}$ & $\mathbf{4 . 5}$ & 3.8 & 3.5 \\
\hline
\end{tabular}

In unembedded contexts and under if, the strongest reading of a punctuated repetition (= (89)a,c) is an 'exactly' reading. Inferences about unpunctuated repetitions are surprisingly weak, possibly because some consultants obtained a reading on which shooting once with a machine gun gave rise to a burst of shots. Here it is particularly informative to consider the 6 responses to 2 open questions, reproduced in (90)-(91). Most answers mention multiple shots and/or a machine

\footnotetext{
${ }^{43}$ Before recording the paradigm, we discussed its acceptability with one of the consultants.
} 
gun (unlike in the case of punctuated repetitions; see the Supplementary Materials for full answers), although not necessarily with an 'at least 4' threshold.

(90) About (87)b: What should the addressee do in the next 10 seconds? (provide a description, not necessarily limited to the number of times the addressee should shoot)

1. Shoot rapidly, at least four times

2. fire a machine gun

3. The addressee should fire a burst of shots. I'm imagining an automatic-style gun, where when you pull the trigger (even without holding it for very long), a burst of several shots (around 3-4) get fired very fast.

4. The addressee should shoot.

5. fire a short burst of shots

6. Shoot rapidly as necessary.

(91) About (87)d: Under what conditions must the speaker give the addressee \$20? (provide a description, not necessarily limited to the number of times the addressee should shoot

1. Provided he fire a number of shots

2. fire two pistols alternatively, one in each hand

3. Minimally, the addressee has to fire a burst of around 4 shots. Beyond that, it depends on more context.

4. if the addressee fires their gun / shoots (not necessarily at a target?)

5. fire a short burst of about four shots

6 . If the player fires at least one burst of shots, or possibly a single shot.

It is worth noting, however, that Consultant 4 apparently obtained an 'at least 1' reading on which the unpunctuated repetition can be satisfied by a single shot. (Consultants also commented on possible effects of intonation, which we did not seek to control for, and differences in realization between one occurrence of the punctuated repetition and the other.) 


\section{Supplementary Materials: Raw Data}

An Excel files with the raw quantitative scores and averages can be downloaded at the following URL (averages have been underlined when there was more than a 2-point difference among the averaged numbers): ${ }^{44}$

https://drive.google.com/file/d/1hs3sBtrkYglQA 0Zka27anIjnWwAwkfy/view?usp=sharing

Raw ASL data can be downloaded in .docx format at the following URL:

https://drive.google.com/file/d/1ekfXy98xINkdOuKdfzdpJy4nMlKmxiQa/view?usp=sharing

The mini-survey on onomatopoeias can be found at the following URL (with links to the audio files):

https://drive.google.com/file/d/1NGRDbyRLYttIrlxAX01V55kCYD9sN0EC/view?usp=sharing

The judgments of 6 linguists (all native speakers of American English, including 1 working on sign language and 1 working on gestures) can be found here:

Anonymized summary: https://drive.google.com/file/d/10WRhYSbISdpbFiQUYMhgR6MIQ8PHXgM /view?usp=sharing

Anonymized Excel file: https://drive.google.com/file/d/IFlhww2Cg5Peeg2vK4vTOxjo0SGf9cszY-/view?usp=sharing

${ }^{44}$ Many thanks to Lucie Ravaux for putting together this Excel files from the scores that appear in the raw data obtained in elicitation sessions. 


\section{References}

Abner, N., Namboodiripad, S., Spaepen, E. \& Goldin-Meadow, S.: 2015, Morphology in Child Homesign: Evidence from Number Marking. (Slides of a talk given at the 2015 Annual Meeting of the Linguistics Society of America, Portland, Oregon)

Bergen, Leon; Levy, Roger; Goodman, Noah D.: 2016, Pragmatic reasoning through semantic inference. Semantics \& Pragmatics, 9. https://semprag.org/article/view/sp.9.20

Byrne RW, Cartmill E, Genty E, Graham KE, Hobaiter C, Tanner J.: 2017, Great ape gestures: intentional communication with a rich set of innate signals. Animal Cognition 20(4):755-69. https://doi.org/10. 1007/s10071-017-1096-4 PMID: 28502063

Chierchia, Gennaro; Fox, Danny; Spector, Benjamin: 2012, Scalar implicature as a grammatical phenomenon. In C. Maienborn, P. Portner, and K. von Heusinger (Eds.), Semantics: An International Handbook of Natural Language Meaning, Volume 3, pp. 2297-2331. de Gruyter.

Cobreros, Pablo; Egré, Paul; Ripley, David; van Rooij, Robert: 2012, Tolerant, Classical, Strict. Journal of Philosophical Logic 41 (2):347-385.

Cohn, Neil: 2013, The Visual Language of Comics: Introduction to the Structure and Cognition of Sequential Images. London: Bloomsbury.

Coppola, M., Spaepen, E., \& Goldin-Meadow, S.: 2013, Communicating about quantity without a language model: Number devices in homesign grammar. Cognitive Psychology, 67, 1-25

Cormier, K.: 2002, Grammaticization of indexic signs: How American Sign Language expresses numerosity. Doctoral dissertation, University of Texas, Austin.

Cremers, Alexandre and Chemla, Emmanuel: 2017, Experiments on the acceptability and possible readings of questions embedded under emotive-factives. Natural Language Semantics, 25(3): 223--261.

Davidson, Kathryn: 2014, Scalar implicatures in a signed language. Sign Language \& Linguistics 17(1). 1-19

Davidson, Kathryn: 2015, Quotation, Demonstration, and Iconicity. Linguistics \& Philosophy 38(6), 477-520.

Davidson, Kathryn and Mayberry, Rachel I.: 2015, Do adults show an effect of delayed first language acquisition when calculating scalar implicatures? Language acquisition 22(4): 329354.

Emmorey, Karen and Herzig, Melissa: 2003, Categorical versus gradient properties of classifier constructions in ASL. In K. Emmorey (Ed.), Perspectives on Classifier Constructions in Signed Languages, Lawrence Erlbaum Associates, Mahwah NJ, pp. 222-246

Feldstein, E.: 2015, The development of grammatical number and space: Reconsidering evidence from child language and homesign through adult gesture. Short manuscript, Harvard University.

Fine, Kit: 1975, Vagueness, Truth and Logic. Synthese 30:265-300.

Fischer, S.: 1973, Two Processes of Reduplication in the American Sign Language. Foundations of Language, 9: 469-480.

Franke, Michael and Jäger, Gerhard: 2016, Probabilistic pragmatics, or why Bayes' rule is probably important for pragmatics. Zeitschrift für Sprachwissenschaft, 35(1), 3-44.

Goodman, Noah D. and Frank, Michael C.: 2016, Pragmatic language interpretation as probabilistic inference. Trends in Cognitive Sciences, 20(11), 818-829.

Graham KE, Hobaiter C, Ounsley J, Furuichi T, Byrne RW: 2018, Bonobo and chimpanzee gestures overlap extensively in meaning. PLoS Biol 16(2):e2004825

Greenberg, Gabriel: 2013, Beyond Resemblance. Philosophical Review, 122, 2

Greenberg, Gabriel: 2014, Reference and Predication in Pictorial Representation. Handout of a talk given at the London Aesthetics Forum (February 19, 2014).

Greenberg, Gabriel: 2019a, Semantics of Pictorial Space. Manuscript, UCLA.

Greenberg, Gabriel: 2019b, Tagging: Semantics at the Iconic/Symbolic Interface. Proceedings of the Amsterdam Colloquium 2020. 
Guerrini, Janek and Migotti, Léo: 2019, Musical gestures in the typology of linguistic inferences. Talk given at the workshop on Linguistic Investigations Beyond Language, Berlin, March 11, 2019.

Hyde, D.: 1997, From Heaps and Gaps to Heaps of Gluts. Mind, 106, 641-660. 14.

Klima, Edward S. and Ursula Bellugi: 1979, The Signs of Language, with Robbin Battison, Penny Boyes Braem, Susan Fischer, Nancy Frishberg, H. Lane, Ella M. Lentz, Don Newkirk, E. L. Newport, C. C. Pedersen, and Patricia Siple. Cambridge, MA: Harvard University Press.

Kuhn, Jeremy: 2015, Cross-categorial singular and plural reference in sign language. Doctoral dissertation, New York University

Kuhn, Jeremy and Aristodemo, Valentina: 2017, Pluractionality, iconicity, and scope in French Sign Language. Semantics \& Pragmatics 10(6).

Liddell, Scott K.: 2003, Grammar, gesture and meaning in American Sign Language. Cambridge: Cambridge University Press.

Nash, David G.: 1986, Topics in Warlpiri Grammar. New York: Garland.

Newman, Paul: 2012, Pluractional verbs: an overview. In Cabredo Hofherr, P. and Laca, B., editors, Verbal plurality and distributivity. De Gruyter, Berlin, Boston.

Nouwen Rick: 2003, Plural pronominal anaphora in context. Doctoral dissertation, Neth. Grad. Sch. Linguist., Univ. Utrecht

Pfau, R. and Steinbach, M.: 2006, Pluralization in sign and in speech: A cross-modal typological study. Linguistic Typology, 10, 49-135

Schlenker, Philippe: 2011, Donkey Anaphora: the View from Sign Language (ASL and LSF). Linguistics and Philosophy, 34(4), 341-395

Schlenker, Philippe: 2014, Iconic Features. Natural Language Semantics 22, 4, 299-356

Schlenker, Philippe: 2019, What is Super Semantics? Philosophical Perspectives 32, 1: 365-453 https://doi.org/10.1111/phpe.12122

Schlenker, Philippe: to appear a, Gestural Grammar. Natural Language \& Linguistic Theory.

Schlenker, Philippe: to appear b, Iconic Presuppositions. Natural Language \& Linguistic Theory.

Schlenker, Philippe and Lamberton, Jonathan: 2019, Iconic Plurality. Linguistics \& Philosophy 42: 45-108 https://doi.org/10.1007/s10988-018-9236-0

Schlenker, Philippe, Lamberton, Jonathan and Santoro, Mirko: 2013, Iconic Variables. Linguistics \& Philosophy 36(2), 91-149

Spector, Benjamin, 2013, Bare Numerals and Scalar Implicatures. Language \& Linguistics Compass, 7(5), 273-294. doi:10.1111/lnc3.12018

van Fraassen, Bas C.: 1966, Singular Terms, Truth Value Gaps and Free Logic. Journal of Philosophy, 63: 481-95

Varzi, Achille: 1995, Supervaluationism and paraconsistency. In D.Batens (ed.), Frontiers in Paraconsistent Logic, pp. 279-297. Baldock: Research Study Press, 2003.

Zucchi, Alessandro: 2011, Event Descriptions and Classifier Predicates in Sign Languages. Presentation at FEAST (Formal and Experimental Advances in Sign language Theory) in Venice, June 21, 2011. 